Cochrane Database of Systematic Reviews

\title{
Very early versus delayed mobilisation after stroke (Review)
}

Langhorne P, Collier JM, Bate PJ, Thuy MNT, Bernhardt J

Langhorne P, Collier JM, Bate PJ, Thuy MNT, Bernhardt J.

Very early versus delayed mobilisation after stroke.

Cochrane Database of Systematic Reviews 2018, Issue 10. Art. No.: CD006187.

DOI: 10.1002/14651858.CD006187.pub3. 
TABLE OF CONTENTS

HEADER

ABSTRACT

PLAIN LANGUAGE SUMMARY

SUMMARY OF FINDINGS

BACKGROUND

OBJECTIVES

METHODS

RESULTS

Figure 1.

Figure 2.

Figure 3.

Figure 4.

Figure 5.

Figure 6.

DISCUSSION

AUTHORS' CONCLUSIONS

ACKNOWLEDGEMENTS

REFERENCES

CHARACTERISTICS OF STUDIES

DATA AND ANALYSES

Analysis 1.1. Comparison 1 Very early mobilisation (VEM) versus standard care (measured at end of scheduled follow-up), Outcome 1 Death or poor outcome.

Analysis 1.2. Comparison 1 Very early mobilisation (VEM) versus standard care (measured at end of scheduled follow-up), Outcome 2 Death.

Analysis 1.3. Comparison 1 Very early mobilisation (VEM) versus standard care (measured at end of scheduled follow-up), Outcome 3 Death or dependence (modified Rankin score 3 to 6).

Analysis 1.4. Comparison 1 Very early mobilisation (VEM) versus standard care (measured at end of scheduled follow-up), Outcome 4 Death or institutional care.

Analysis 1.5. Comparison 1 Very early mobilisation (VEM) versus standard care (measured at end of scheduled follow-up), Outcome 5 Activities of daily living (ADL) score.

Analysis 1.6. Comparison 1 Very early mobilisation (VEM) versus standard care (measured at end of scheduled follow-up), Outcome 6 Subjective Health Status score.

Analysis 1.7. Comparison 1 Very early mobilisation (VEM) versus standard care (measured at end of scheduled follow-up), Outcome 7 Able to walk.

Analysis 1.8. Comparison 1 Very early mobilisation (VEM) versus standard care (measured at end of scheduled follow-up), Outcome 8 Mobility score.

Analysis 1.9. Comparison 1 Very early mobilisation (VEM) versus standard care (measured at end of scheduled follow-up), Outcome 9 Any complication: participants who experienced at least one complication.

Analysis 1.10. Comparison 1 Very early mobilisation (VEM) versus standard care (measured at end of scheduled follow-up), Outcome 10 Type of complication: participants who experienced at least one complication.

Analysis 1.11. Comparison 1 Very early mobilisation (VEM) versus standard care (measured at end of scheduled follow-up), Outcome 11 Mood score.

Analysis 1.12. Comparison 1 Very early mobilisation (VEM) versus standard care (measured at end of scheduled follow-up), Outcome 12 Length of acute hospital stay (days).

Analysis 2.1. Comparison 2 Very early mobilisation versus standard care (results at 3 months), Outcome 1 Death or poor outcome.

Analysis 2.2. Comparison 2 Very early mobilisation versus standard care (results at 3 months), Outcome 2 Death.

Analysis 2.3. Comparison 2 Very early mobilisation versus standard care (results at 3 months), Outcome 3 Death or dependence (modified Rankin score 3 to 6).

Analysis 2.4. Comparison 2 Very early mobilisation versus standard care (results at 3 months), Outcome 4 Death or institutional care.

Analysis 2.5. Comparison 2 Very early mobilisation versus standard care (results at 3 months), Outcome 5 Activities of daily living (ADL) score.

ADDITIONAL TABLES 
[Intervention Review]

\section{Very early versus delayed mobilisation after stroke}

Peter Langhorne ${ }^{1}$, Janice M Collier ${ }^{2}$, Patricia J Bate ${ }^{3}$, Matthew NT Thuy ${ }^{4}$, Julie Bernhardt ${ }^{5}$

${ }_{1}^{1}$ Academic Section of Geriatric Medicine, ICAMS, University of Glasgow, Glasgow, UK. 2Very Early Rehabilitation Stroke Research Program, National Stroke Research Institute, Heidelberg Heights, Australia. ${ }^{3}$ Seacliff, Australia. ${ }^{4}$ National Stroke Research Institute, Austin Health, Heidelberg Heights, Australia. ${ }^{5}$ Florey Institute of Neuroscience and Mental Health, Heidelberg, Australia

Contact address: Julie Bernhardt, Florey Institute of Neuroscience and Mental Health, 245 Burgundy Street, Heidelberg, Victoria, 3081, Australia.j.bernhardt@unimelb.edu.au.

Editorial group: Cochrane Stroke Group.

Publication status and date: New search for studies and content updated (no change to conclusions), published in Issue 10, 2018.

Citation: Langhorne P, Collier JM, Bate PJ, Thuy MNT, Bernhardt J. Very early versus delayed mobilisation after stroke. Cochrane Database of Systematic Reviews 2018, Issue 10. Art. No.: CD006187. DOI: 10.1002/14651858.CD006187.pub3.

Copyright @ 2018 The Cochrane Collaboration. Published by John Wiley \& Sons, Ltd.

\section{A B S T R A C T}

\section{Background}

Very early mobilisation (VEM) is performed in some stroke units and recommended in some acute stroke clinical guidelines. However, it is unclear whether very early mobilisation independently improves outcome after stroke.

\section{Objectives}

To determine whether very early mobilisation (started as soon as possible, and no later than 48 hours after onset of symptoms) in people with acute stroke improves recovery (primarily the proportion of independent survivors) compared with usual care.

\section{Search methods}

We searched the Cochrane Stroke Group Trials Register (last searched 31 July 2017). We also systematically searched 19 electronic databases including; CENTRAL; 2017, Issue 7 in the Cochrane Library (searched July 2017), MEDLINE Ovid (1950 to August 2017), Embase Ovid (1980 to August 2017), CINAHL EBSCO (Cumulative Index to Nursing and Allied Health Literature; 1937 to August 2017 ), PsycINFO Ovid (1806 to August 2017), AMED Ovid (Allied and Complementary Medicine Database), SPORTDiscus EBSCO (1830 to August 2017 ). We searched relevant ongoing trials and research registers (searched December 2016), the Chinese medical database, Wanfangdata (searched to November 2016), and reference lists, and contacted researchers in the field.

\section{Selection criteria}

Randomised controlled trials (RCTs) of people with acute stroke, comparing an intervention group that started out-of-bed mobilisation within 48 hours of stroke, and aimed to reduce time-to-first mobilisation, with or without an increase in the amount or frequency (or both) of mobilisation activities, with usual care, where time-to-first mobilisation was commenced later.

\section{Data collection and analysis}

Two review authors independently selected trials, extracted data, assessed risk of bias, and applied the GRADE approach to assess the quality of the evidence. The primary outcome was death or poor outcome (dependency or institutionalisation) at the end of scheduled follow-up. Secondary outcomes included death, dependency, institutionalisation, activities of daily living (ADL), extended ADL, quality of life, walking ability, complications (e.g. deep vein thrombosis), patient mood, and length of hospital stay. We also analysed outcomes at three-month follow-up.

\section{Main results}

We included nine RCTs with 2958 participants; one trial provided most of the information (2104 participants). The median (range) delay to starting mobilisation after stroke onset was 18.5 (13.1 to 43) hours in the VEM group and 33.3 (22.5 to 71.5 ) hours in the usual care group. 
The median difference within trials was 12.7 (4 to 45.6) hours. Other differences in intervention varied between trials; in five trials, the VEM group were also reported to have received more time in therapy, or more mobilisation activity.

Primary outcome data were available for 2542 of 2618 (97.1\%) participants randomized and followed up for a median of three months. VEM probably led to similar or slightly more deaths and participants who had a poor outcome, compared with delayed mobilisation (51\% versus 49\%; odds ratio (OR) 1.08, 95\% confidence interval $(\mathrm{Cl}) 0.92$ to $1.26 ; \mathrm{P}=0.36 ; 8$ trials; moderate-quality evidence). Death occurred in $7 \%$ of participants who received delayed mobilisation, and $8.5 \%$ of participants who received VEM (OR $1.27,95 \% \mathrm{Cl} 0.95$ to $1.70 ; \mathrm{P}=0.11$; 8 trials, 2570 participants; moderate-quality evidence), and the effects on experiencing any complication were unclear (OR 0.88 ; $95 \% \mathrm{Cl}$ 0.73 to $1.06 ; \mathrm{P}=0.18 ; 7$ trials, 2778 participants; low-quality evidence). Analysis using outcomes collected only at three-month follow-up did not alter the conclusions.

The mean ADL score (measured at end of follow-up, with the 20-point Barthel Index) was higher in those who received VEM compared with the usual care group (mean difference (MD) 1.94, 95\% Cl 0.75 to 3.13, $\mathrm{P}=0.001$; 8 trials, 9 comparisons, 2630/2904 participants (90.6\%); lowquality evidence), but there was substantial heterogeneity (93\%). Effect sizes were smaller for outcomes collected at three-month followup, rather than later.

The mean length of stay was shorter in those who received VEM compared with the usual care group ( $\mathrm{MD}-1.44,95 \% \mathrm{Cl}-2.28$ to $-0.60, \mathrm{P}=$ $0.0008 ; 8$ trials, 2532/2618 participants (96.7\%); low-quality evidence). Confidence in the answer was limited by the variable definitions of length of stay. The other secondary outcome analyses (institutionalisation, extended activities of daily living, quality of life, walking ability, patient mood) were limited by lack of data.

Sensitivity analyses by trial quality: none of the outcome conclusions were altered if we restricted analyses to trials with the lowest risk of bias (based on method of randomization, allocation concealment, completeness of follow-up, and blinding of final assessment), or information about the amount of mobilisation.

Sensitivity analysis by intervention characteristics: analyses restricted to trials where the mean VEM time-to-first mobilisation was less than 24 hours, showed an odds of death of $1.35\left(95 \% \mathrm{Cl} 0.99\right.$ to $1.83 ; \mathrm{P}=0.06 ; \mathrm{I}^{2}=25 \% ; 5$ trials). Analyses restricted to the trials that clearly reported a more prolonged out-of-bed activity showed a similar primary outcome (OR 1.14; 0.96 to $1.35 ; \mathrm{P}=0.13 ; \mathrm{I}^{2}=28 \% ; 5$ trials), and odds of death (OR $1.27 ; 0.93$ to $1.73 ; P=0.13 ; 1^{2}=0 \% ; 4$ trials) to the main analysis.

Exploratory network meta-analysis (NMA): we were unable to analyze by the amount of therapy, but low-quality evidence indicated that time-to-first mobilisation at around 24 hours was associated with the lowest odds of death or poor outcome, compared with earlier or later mobilisation.

\section{Authors' conclusions}

VEM, which usually involved first mobilisation within 24 hours of stroke onset, did not increase the number of people who survived or made a good recovery after their stroke. VEM may have reduced the length of stay in hospital by about one day, but this was based on low-quality evidence. Based on the potential hazards reported in the single largest RCT, the sensitivity analysis of trials commencing mobilisation within 24 hours, and the NMA, there was concern that VEM commencing within 24 hours may carry an increased risk, at least in some people with stroke. Given the uncertainty around these effect estimates, more detailed research is still required.

\section{PLAIN LANGUAGE SUMMARY}

\section{Very early versus delayed mobilisation after stroke}

\section{Review question}

Does very early and active mobilisation improve recovery after stroke compared with more delayed mobilisation?

\section{Background}

Care in a stroke unit is recommended for people soon after a stroke, and results in an improved chance of surviving, returning home, and regaining independence. Very early mobilisation (helping people to get up out of bed very early, and more often after the onset of stroke symptoms) is performed in some stroke units, and is recommended in many acute stroke clinical guidelines. However, the impact of very early mobilisation on recovery after stroke is not clear.

\section{Search date}

This review is up-to-date to July 2017.

\section{Study characteristics}

This review identified nine trials (2958 participants), although one trial (2104 participants) provided most of the information. On average, very early mobilisation participants started mobilisation 18.5 hours after their stroke, compared with 33.3 hours in the usual care group. In five trials, the very early mobilisation group were also known to have spent more time per day in therapy, or participated in a mobilisation activity.

\section{Main results}


Very early mobilisation did not increase the number of people who survived or made a good recovery after their stroke. There was a suggestion that very early mobilisation may reduce the length of stay in hospital by about one day. However, results from the single largest trial, and from an analysis of trials that started mobilising participants very early, raised the concern that starting intensive mobilisation within 24 hours of stroke may carry some increased risk, at least for some people with stroke. This potential risk needs to be clarified.

\section{Quality of the evidence}

Overall, the main results were supported by moderate-quality evidence overall, but low-quality evidence backed length of hospital stay and activities of daily living. 
SUMMARY OF FINDINGS

\section{Summary of findings for the main comparison.}

Very early mobilisation versus delayed mobilisation

Patient or population: adults with acute stroke

Settings: stroke unit or acute ward

Intervention: very early mobilisation (VEM)

Comparison: delayed mobilisation

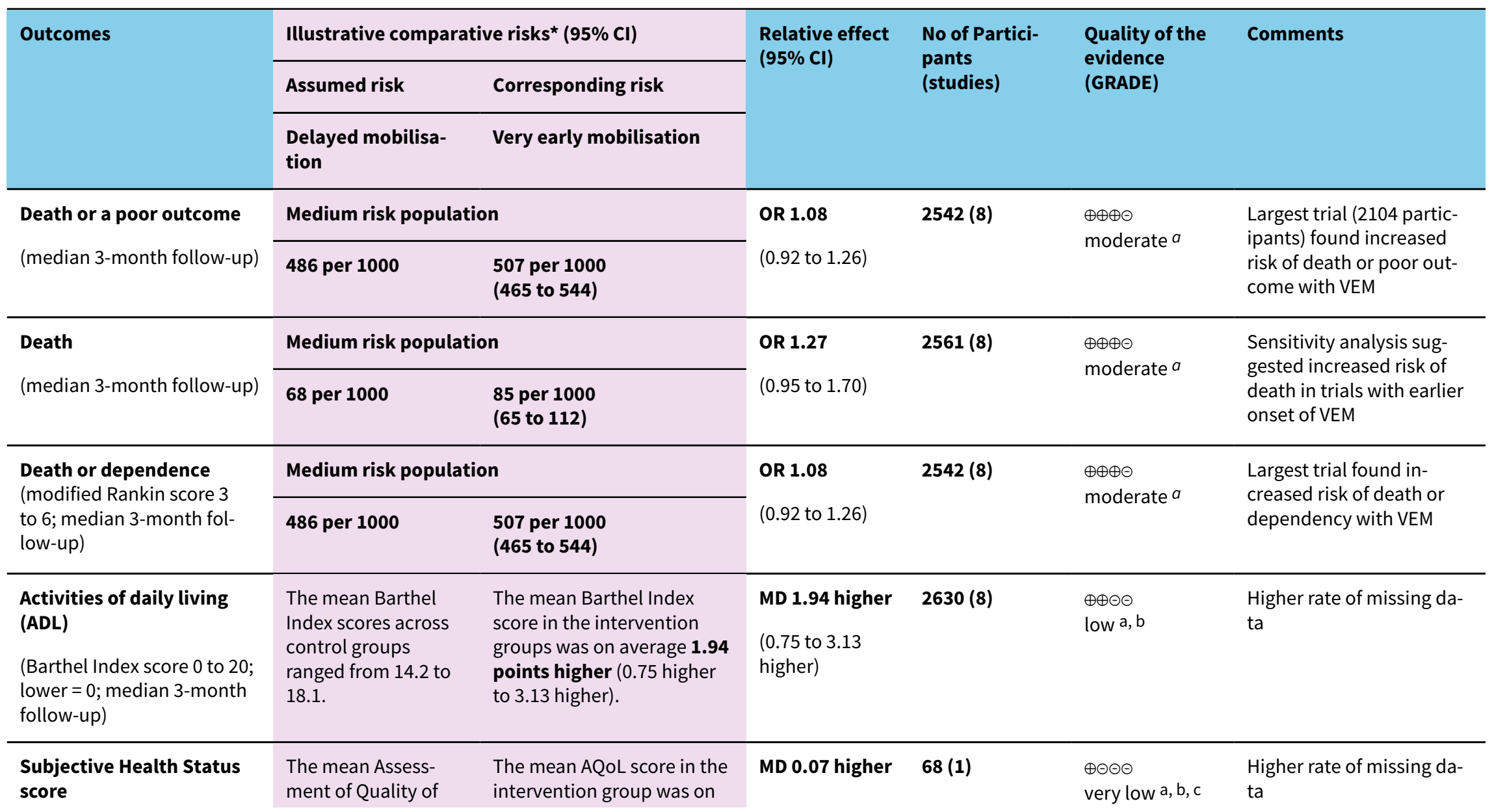


Any complication: partic-

ipants who experience at

Medium risk population

OR 0.88

2778 (6)

$\oplus \oplus \oplus \odot$

Uncertain blinding at fol-

least one complication

(median 3-month follow-up)

224 per 1000

200 per 1000

(0.73 to 1.06 )

Length of acute hospital

stay (days)

The mean length of
stay across control
groups ranged from
9.8 to 14.9 days.

The mean length of stay

in the intervention groups

was, on average, $\mathbf{1 . 4 4}$ days

less ( 2.28 days less to 0.60

day less)

\section{1.44 lower}

2551 (8)

(2.28 lower to

0.60 lower)

low a, c low-up

(2)

$\oplus \oplus \ominus \ominus$

low a, b

Different definitions and

imprecise measures of

length of stay were report-

ed

Result largely depends on two small trials with small SDs

*The basis for the assumed risk (e.g. the mean control group risk across studies) is provided in footnotes. The corresponding risk (and its $95 \%$ confidence interval) is based on the assumed risk in the comparison group and the relative effect of the intervention (and its $95 \% \mathrm{Cl}$ ).

Cl: Confidence interval; OR: odds ratio; MD: mean difference

GRADE Working Group grades of evidence

High quality: Further research is very unlikely to change our confidence in the estimate of effect.

Moderate quality: Further research is likely to have an important impact on our confidence in the estimate of effect and may change the estimate.

Low quality: Further research is very likely to have an important impact on our confidence in the estimate of effect and is likely to change the estimate.

Very low quality: We are very uncertain about the estimate.

a Downgraded for potential risk of performance bias.

$b$ Downgraded for unexplained heterogeneity.

c Downgraded for imprecision. 


\section{B A C K G R O U N D}

\section{Description of the condition}

Stroke presents a major global public health challenge, with over five million people dying from stroke each year, and many more living with chronic disability (Murray 2012). We know that treatment in an organised multidisciplinary stroke unit (compared with treatment in a general medical ward) reduces the odds of being dead or disabled at 12 months post stroke (SUTC 2013). However, relatively little is known about which components of acute stroke unit care may be responsible for better outcomes (Langhorne 2012). Early rehabilitation (in particular early mobilisation) is described as an important feature of stroke unit care, but there is only limited information about what early rehabilitation entails and who provides it (Langhorne 2012). In addition to the uncertainties surrounding the optimal amount of rehabilitation that can be provided early after stroke, exactly how early rehabilitation should start is controversial.

\section{Description of the intervention}

Early mobilisation (getting people up and out of bed and sitting, standing, and walking early after stroke) is an established feature of acute stroke unit care, particularly in many Scandinavian hospitals (Indredavik 1999). In other parts of the world, patients may be restricted to bed for some days before mobilisation is allowed (Diserens 2006). In some cases, these differences in practice reflect concerns about the possibility that early mobilisation may have a detrimental effect on the vulnerable ischaemic penumbra (Diserens 2006), although there is little evidence to support this view (Bernhardt 2007; Bernhardt 2015), while in other cases, they are likely to reflect historical practices.

\section{How the intervention might work}

The biological rationale for early mobilisation is based on three lines of argument: 1 ) there is evidence that bedrest has a harmful impact across many conditions, and is likely to slow recovery (Allen 1999; Mutin-Carino 2014); 2) some of the most common and serious complications after stroke are those related to immobility (Langhorne 2000); and 3) current concepts of biological recovery after brain injury suggest a narrow window of opportunity for brain plasticity and repair (Murphy 2009). We know that the routine day of most acute stroke patients is largely inactive, so introducing frequent training out of bed could reduce the risk of complications of immobility (Bernhardt 2004; Bernhardt 2015). Also, if the brain does indeed remodel itself based on experience (Johansson 2000; Krakauer 2012), then early task-specific training may well contribute to improving recovery (Pekna 2012).

However, there are also concerns about potential harm of early mobilisation, particularly in the first 24 hours after stroke onset (Bernhardt 2004; Skarin 2011). These concerns include haemodynamic considerations: whether raising the person's head early after stroke will impair cerebral blood flow and cerebral perfusion (Skarin 2011). Alternatively, in the case of intracerebral haemorrhage, whether early mobilisation will increase the risk of inducing further bleeding (Olavarria 2014). As a result of these theoretical concerns, some clinicians have advocated initial bedrest for people with stroke (Skarin 2011). This uncertainty about the best approach stimulated a large cluster-randomised trial that explored the impact of head postioning in people with acute stroke (Muñoz-Venturelli 2015). This trial found no clear difference in outcomes for people nursed flat for the first 24 hours after stroke versus those nursed with their head up, and allowed to mobilise to the toilet (Anderson 2017).

\section{Why it is important to do this review}

Although very early mobilisation (VEM) has been recommended in a number of acute stroke clinical guidelines (Adams 2003; Bernhardt 2015; NSF 2007), only indirect evidence currently supports these recommendations (Indredavik 1999). It is not known whether VEM independently improves outcome after stroke.

\section{O B J E C T IVES}

To determine whether very early mobilisation (started as soon as possible, and no later than 48 hours after onset of symptoms) in people with acute stroke improves recovery (primarily the proportion of independent survivors) compared with usual care.

\section{METHODS}

\section{Criteria for considering studies for this review Types of studies}

We sought all randomized trials, with or without blinding, of VEM within 48 hours of symptom onset compared with more delayed, usual care (i.e. normal practice or no routine intervention).

\section{Types of participants}

Participants in trials had to be adults with a definite clinical diagnosis of stroke (focal neurological deficit of cerebrovascular origin), and could be mobilised within 48 hours of stroke onset. There were no age restrictions.

\section{Types of interventions}

We defined VEM as any intervention delivered with the aim of reducing the time from stroke onset to first mobilisation (first outof-bed episode), which may have included increasing the amount of out-of-bed physical activity (e.g. participation in activities of daily living (ADLs), such as walking to the toilet, transferring on and off the toilet, sitting out of bed, standing, and walking). Any form of VEM was considered, regardless of the number and discipline of staff assisting, and the dose or duration of the intervention. We defined usual care as the usual mobilisation practice.

\section{Types of outcome measures}

The aim was to identify all outcomes of interest at the end of scheduled follow-up, and also (where available) at three-month follow-up.

\section{Primary outcomes}

Death or a poor outcome: the number of participants who died or had a poor outcome at the end of scheduled follow-up (in preferred order, this was defined as: remained dependent (modified Rankin Score (mRS) 3 to 5 , or Barthel score $<15$, or equivalent), or required admission to institutional care. We defined institutional care as care provided in a residential home, nursing home, or hospital at followup.

\section{Secondary outcomes}

- Death: number of deaths from any cause 
- Death or dependence: the number of participants dead or physically dependent (mRS 3 to 5 , or equivalent)

- Death or requiring institutional care: we defined institutional care as care provided in a residential home, nursing home, or hospital at follow-up.

- Performance in activities of daily living (ADL): using a recognised ADL score

- Performance in extended activities of daily living (community and domestic activities): using a recognised extended ADL score

- Patient subjective health status or quality of life score

- Ability to walk: walking unassisted (without help from another person), reported alone, or as a component of a functional mobility scale

- Mobility score: using a recognised mobility score

- Complications (adverse events): number or severity (or both) of complications (adverse events), including deep vein thrombosis (DVT), pulmonary embolism (PE), incidence and grade of pressure sores (using standardized grading scale), number of incontinent episodes over 24 hours, severity of incontinence, chest infection, falls, and physiological variables (blood pressure, oxygen, temperature) recorded

- Type of complication (adverse events): categorised as complications of immobility (DVT, PE, incidence and grade of pressure sores (using standardized grading scale), chest infection, urinary tract infection, falls), and other complications

- Patient mood: using a recognised measure of mood

- Length of stay in acute hospital (recorded in days)

\section{Search methods for identification of studies}

See the Cochrane Stroke Group Trials Register. We aimed to identify all relevant RCTs, regardless of language or publication status (published, unpublished, in press, or in progress). We arranged translation of relevant articles where required.

\section{Electronic searches}

We searched the Cochrane Stroke Group Trials Register, which was last searched by the Cochrane Stroke Group Information Specialist on 31 July 2017. In addition, we systematically searched the following electronic databases.

- Cochrane Central Register of Controlled Trials (CENTRAL; 2017, Issue 7) in the Cochrane Library (searched August 2017; Appendix 1);

- MEDLINE Ovid (1950 to August 2017; Appendix 2);

- Embase Ovid (1980 to August 2017; Appendix 3);

- CINAHL EBSCO (Cumulative Index to Nursing and Allied Health Literature; 1937 to August 2017; Appendix 4);

- PsycINFO Ovid (1806 to August 2017; Appendix 5);

- AMED Ovid (Allied and Complementary Medicine Database; 1985 to August 2017; Appendix 6);

- SPORTDiscus EBSCO (1830 to August 2017; Appendix 7);

- Science Citation Index Expanded (1900 to August 2017; Appendix 8);

- Social Sciences Citation Index (1956 to August 2017);

- Arts and Humanities Citation Index (1975 to August 2017);

- PEDro (Physiotherapy Evidence Database (www.pedro.fhs.usyd.edu.au/; 1929 to December 2006);
- REHABDATA (National Rehabilitation Information Centre (www.naric.com/?q=en/REHABDATA; 1956 to November 2016);

- CIRRIE (Center for International Rehabilitation Research Information and Exchange (cirrie.buffalo.edu/; 1990 to November 2016);

- OTSeeker (searched to November 2016);

- Wan Fang Database (www.wanfangdata.com/; searched to November 2016);

- British Association of Occupational Therapists' Library Collection (including Thesis Collection; searched to November 2016);

- ProQuest Dissertations and Theses - Global (formerly Index to UK Theses; searched to November 2016).

We used the search strategy for MEDLINE, and with the assistance of the Cochrane Stroke Group Information Specialist, modified it to suit other databases (Appendix 2).

We searched the following ongoing trials and research registers.

- US National Institutes of Health Ongoing Trials Register ClinicalTrials.gov (ClinicalTrials.gov; searched December 2016);

- World Health Organization (WHO) International Clinical Trials Registry Platform (ICTRP'; apps.who.int/trialsearch; searched December 2016);

- ISRCTN Registry (www.isrctn.com; formerly the Meta Register of Controlled Trials (mRCT): www.controlled-trials.com/mrct/; searched December 2016);

- UK Department of Health Research Findings Register (ReFeR and latterly www.isrctn.com/).

\section{Searching other resources}

- We originally handsearched all available years of the following journals (these were not updated after 2006 in view of the increased sensitivity of our other search strategies).

* Advances in Occupational Medicine and Rehabilitation (1996 to 1999);

* Advances in Clinical Neurosciences and Rehabilitation (2001 to 2006);

* Advances in Clinical Rehabilitation (1987 to 1990);

* Archives of Occupational Therapy (renamed Occupational Therapy and Rehabilitation; 1922 to 2006);

* Canadian Journal of Rehabilitation (1987 to 1999);

* Chinese Journal of Physical Medicine and Rehabilitation (1980 to 2006);

* European Journal of Physical Medicine and Rehabilitation (1991 to 1999);

* International Journal of Rehabilitation and Health (1995 to 2000);

* Journal of Rehabilitation Administration (1987 to 2006);

* Rehabilitation (1948 to 1949, 1951 to 1977);

* Rehabilitation in Canada (1963 to 1972);

* Rehabilitation Nursing Research (1992 to 1996);

* Topics in Stroke Rehabilitation (1995 to 2006).

- We sought information about unpublished or incomplete trials via correspondence with researchers or organisations (or both), known to be involved in previous relevant studies. 
- We checked the bibliographies of included studies and relevant reviews for further references to additional relevant trials.

\section{Data collection and analysis}

\section{Selection of studies}

We selected trials for inclusion based on the described inclusion criteria. For this update, one review author (PL) read all the references identified and eliminated any obviously irrelevant studies. Two review authors (selected from MT, JMC, PL, JB, TB) independently read the titles (and abstracts if available) of the identified references. We obtained the full text for remaining studies, and based on the inclusion criteria (types of studies, types of participants, aims of interventions, outcome measures), two review authors (selected from MT, JMC, PL, JB, TB) independently classified these as eligible, not eligible, or unsure. We excluded any trials that both review authors classified as not eligible. At least two review authors (selected from MT, JMC, PL, JB, TB) independently made decisions about inclusion, and we resolved differences in opinion regarding trial eligibility by discussion between all review authors. If further information was needed to reach consensus, we contacted trialists, and attempted to obtain the missing information. We arranged trial selection decisions to avoid trialists making decisions about trials in which they were involved.

Papers and abstracts in Chinese were reviewed by one researcher fluent in Chinese and with medical training (YL, JW, or WZ, see Acknowledgements). One review author (MT or PL) assessed a short English description of the decision made.

\section{Data extraction and management}

Our primary aim was to obtain standardized data through collaboration with the original trialists. Two review authors (from MT, JMC, PL, TB) independently extracted data from published sources, using a standard data recording form. We extracted important risk of bias indicator data such as concealment of randomization, blinding of outcome evaluation, and intention-totreat analysis, and graded these as present, absent, or unclear. In addition, we extracted data relating to all primary and secondary outcomes of interest, as well as important imbalances in prognostic factors, comparison (details of the intervention in the treatment and control groups, details of co-intervention(s) in both groups), and other relevant outcomes not prespecified in the protocol (for example, exertion).

The review authors checked all the extracted data for agreement; a third review author arbitrated any items where they could not reach consensus. If necessary, we contacted trialists to request more information, clarification, or missing data.

\section{Assessment of risk of bias in included studies}

Two review authors (from PL, MT or TB), who had no involvement in the study under review, independently evaluated the risk of bias of included trials, using the Cochrane 'Risk of bias' tool, and extracted information for each included trial about the method of randomization and allocation concealment, blinding of outcome assessment, and any intention-to-treat analyses. We applied the GRADE approach to assess the quality of the evidence (Higgins 2011).

\section{Measures of treatment effect}

We compared interventions that commenced mobilisation earlier and aimed to improve the frequency or amount (or both) of mobilisation activity, delivered by any member of the acute stroke unit staff, versus more delayed mobilisation (usual care) on primary and secondary outcome measures. We analysed binary (dichotomous) outcomes with a fixed-effect model, as odd ratios (OR) with 95\% confidence intervals (CI). For continuous outcomes, we calculated a mean difference (MD) with a random-effects model, to take account of any statistical heterogeneity. We used the standardized mean difference (SMD) where different scales were used for the same outcome.

\section{Unit of analysis issues}

We only included randomized parallel group trials, in which the unit of analysis was the individual participant. We did not include cluster- or cross-over RCTs.

\section{Dealing with missing data}

We assessed the degree of missing data for each of the main outcomes and had planned to conduct sensitivity analyses if more than $15 \%$ of randomized participant data were missing.

\section{Assessment of heterogeneity}

We examined the statistical heterogeneity between studies using the $I^{2}$ statistic. We determined substantial heterogeneity as a value greater than $50 \%$.

\section{Assessment of reporting biases}

We had planned to carry out funnel plots if more that 10 trials were available.

\section{Data synthesis}

If continuous data were only available as medians and interquartile ranges, we estimated the mean using an established method (Wan 2014). Where only interquartile ranges (IQR) were reported, we inferred the standard deviation as follows: the IQR will incorporate $50 \%$ of the distribution of data compared with standard deviation, which can be expected to include $70 \%( \pm 35 \%)$ of the distribution. Therefore, assuming a normal distribution, one standard deviation should equal the IQR/( $2 \times 0.7)$. We used the Cochrane Review Manager 5 software for analyses (RevMan 2014).

\section{Subgroup analysis and investigation of heterogeneity}

We did not plan subgroup analyses for this version of the review, which did not have individual patient data available. We planned to explore heterogeneity through sensitivity analyses.

\section{Sensitivity analysis}

We conducted sensitivity analyses to evaluate the effect of differences in methodological quality (method of randomization, allocation concealment, intention-to-treat analysis, and blinding of final assessment), time-to-first mobilisation, and amount of mobilisation activity.

\section{Network meta-analysis}

In view of new approaches to meta-analysis, we also included a post-hoc network meta-analysis (NMA) of trial data. This review aimed to include trials that compared the effect of a shorter 
time-to-first mobilisation (with or without an increase in the amount or frequency (or both) of mobilisation activities) with usual care (where time-to-first mobilisation started later). However we expected that within this broad definition, the included trials would comprise a range of treatment comparisons. Therefore, we included an exploratory NMA to explore, where possible, the impact of different treatment characteristics (time-to-first mobilisation and amount of mobilisation activity). We used Metainsight software, designed specifically for this role, to conduct our NMA (https://crsu.shinyapps.io/metainsightb/).

A NMA uses information from both direct and indirect estimates of treatment effect (Tonin 2017). Direct estimates are provided by a head-to-head comparison (e.g. treatment A versus treatment B). Indirect estimates are provided by two or more head-to-head comparisons that share a common comparator (e.g. when A verus $B$ is the comparison of interest, then use trials with $A$ versus $C$ and with $B$ versus $C$ ). A network is then formed, using a collection of trials that allow, through direct and indirect comparisons, calculation of the relative effects of all treatments versus each other (or versus a single comparator).

A key assumption in NMA is the transitivity (or similarity) assumption that concerns the validity of making indirect comparisons. This assumes that treatment effects are 'exchangeable' across the included trials and all treatments are 'jointly randomisable'. In other words, all treatment categories could feasibly be randomized in the same trial and those that are not treatment arms in any given trial are 'missing at random' (Lu 2006). This assumption cannot be formally tested statistically, and it must be judged through careful consideration of the trial settings and characteristics, treatment mechanisms, and participant demographics to investigate if any differences would be expected to modify relative treatment effects.

A second key assumption (known as the consistency assumption) assumes that it is feasible to make indirect comparisons between two treatments, and that the indirect evidence is consistent with the direct evidence, where such a comparison exists (Lu 2006). The consistency assumption is evaluated statistically by comparing the difference between the direct and the indirect estimate for each loop of evidence. Therefore, we examined for any important differences in numerical results between direct, indirect, and network results.

\section{Assessing the quality of the evidence}

NMA presents challenges when grading the quality of evidence. We used the approach of the GRADE group as follows (Puhan 2014).

- Present direct and indirect treatment estimates for each comparison of the evidence network.

- Rate the quality of each direct and indirect effect estimate (downgrading for risk of bias, inconsistency, indirectness, imprecision, and publication bias).

- Present the NMA estimate for each comparison of the evidence network.

- Rate the quality of each NMA effect estimate (as above).

\section{RESULTS}

\section{Description of studies}

Please see Characteristics of included studies, Characteristics of ongoing studies, Characteristics of studies awaiting classification and Characteristics of excluded studies.

\section{Results of the search}

The collated searches from this, and the previous version of the review, identified 21,395 titles (Bernhardt 2009; Figure 1). The original version of the review identified a total of 39 trials of interest by September 2007, 28 of which we excluded; 10 were ongoing or unclassified, and one was included (AVERT II 2008). 


\section{Figure 1. Study flow diagram}

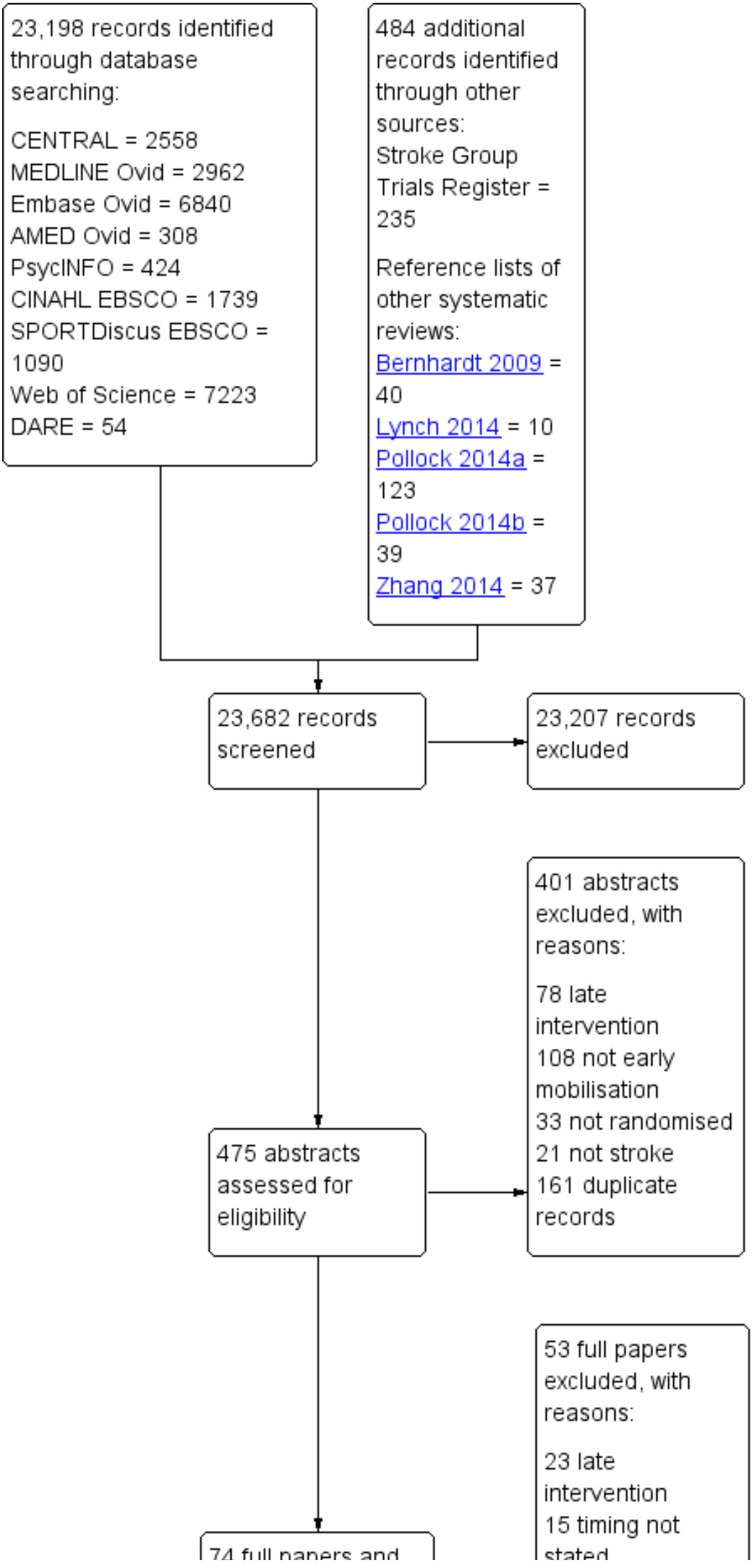


Figure 1. (Continued)

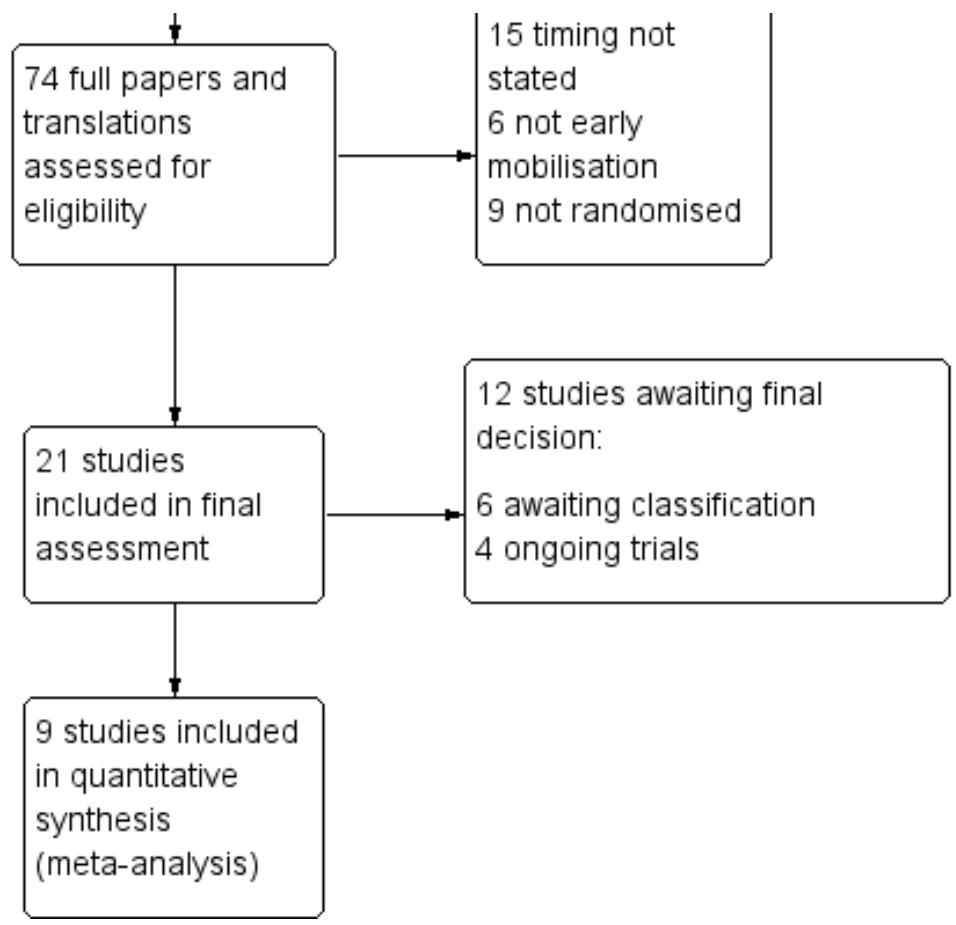

This update identified a total of 74 studies of interest, 53 of which we excluded for various reasons, as detailed in the 'Characteristics of excluded studies' table.

In addition to the excluded studies, there are eight studies for which we have been unable to obtain full references and for which we have been unsuccessful in obtaining any further information from the authors, therefore they are still awaiting classification (Izumi 2001; Liu 2010e; Nilsson 2003; Skevin 2009; Song 2010; Xu 2001; Zheng 2004a; Zielke 2003). Three trials are currently ongoing, with no available data (ChiCTR-ICR-15005992; ChiCTR-IPR-16008652; ChiCTR-TRC-08000201). They plan to recruit about 400 participants in total. One trial is in the development stage (AVERT-DOSE 2017). Details of these trials are reported in the 'Characteristics of ongoing studies' table.

\section{Included studies}

We included nine trials in the review (AVERT II 2008; AVERT II 2015; Chippala 2015a; Chippala 2015b; Langhorne 2010; Morreale 2016; Poletto 2015; SEVEL 2016; Sundseth 2012). Note that the 'Characteristics of included studies' table contains 11 items, because one trial contained two arms (Morreale 2016), which for some analyses had to be analysed separately (Morreale 2016 CTE; Morreale 2016 PNF).

The nine included trials had a total of 2958 participants; the inclusion and exclusion criteria are shown in the 'Characteristics of included studies' table. The included participants were broadly representative of the stroke population, although the numbers were dominated by one multicentre trial of 2104 participants (AVERT III 2015). Across all nine included trials, the mean age of participants within each trial ranged from 60 to 77 years (median 68 ), and the proportion of males ranged from $35 \%$ to $72 \%$ (median $52 \%)$. Stroke severity was measured using the National Institutes of Health Stroke Scale (NIHSS), and usually classified as mild (NIHSS 0 to 7), moderate (NIHSS 8 to 16 ), or severe (NIHSS > 16) stroke (Brott 1989). The baseline stroke severity was typically in the moderate range, with the proportion with intracerebral haemorrhage ranging from $0 \%$ to $20 \%$ (median $12 \%$ ).

We did not observe imbalances between groups in important prognostic factors, including age, stroke severity, type of stroke, and premorbid disability.

We categorised the intervention details using the TIDieR classification, which are summarized in the 'Characteristics of included studies' table (Hoffman 2014). The interventions were delivered in a stroke unit (AVERT II 2008; AVERT III 2015; Chippala 2015a; Chippala 2015b; Langhorne 2010; Poletto 2015; Sundseth 2012), stroke centre ( SEVEL 2016), or neurology ward (Morreale 2016). The very early mobilisation (VEM) intervention was provided by physiotherapy, with or without nursing staff, and continued while the participant was in hospital. The nine trials all tested different versions of a VEM strategy as follows.

\section{Earlier onset}

In six trials, intervention participants were mobilised within 24 hours of stroke (AVERT II 2008; AVERT III 2015; Chippala 2015a; Chippala 2015b; Morreale 2016; Sundseth 2012), while in three, this usually occurred at 24 to 48 hours (Langhorne 2010; Poletto 2015; SEVEL 2016). Across all trials, the median (range) delay to starting mobilisation after stroke was 18.5 (13.1 to 43 ) hours in the VEM group, and 33.3 (22.5 to 71.5 ) hours in the usual care group. The median difference (range) within trials was 12.7 (4 to 45.6) hours.

\section{More intensive}

In four trials, VEM included a recorded increase in time of out-of-bed mobility activities (AVERT II 2008; AVERT III 2015; Langhorne 2010; Poletto 2015). One trial reported a longer time 
participants were recorded sitting out of bed, but not a very active mobilisation activity (SEVEL 2016). One trial reported a similar intensity (Sundseth 2012), but information was more limited from three others (Chippala 2015a; Chippala 2015b; Morreale 2016).

\section{Modifiable intervention}

Five trials reported that the intervention could be modified or stopped in the event of a deterioration in the participant's physiological or neurological status (AVERT II 2008; AVERT III 2015; Langhorne 2010; SEVEL 2016; Sundseth 2012). Information was more limited from four others (Chippala 2015a; Chippala 2015b; Morreale 2016; Poletto 2015).

\section{Monitored intervention}

In several trials, the difference in care delivered was timed and recorded. Five trials recorded an earlier onset of mobilisation with an increased number of minutes of out-of-bed activity (AVERT II 2008; AVERT III 2015; Langhorne 2010; Poletto 2015; SEVEL 2016). Three trials recorded an earlier onset of mobilisation, but no reported difference in activity (Chippala 2015a; Chippala 2015b; Sundseth 2012). We had limited information from one other trial (Morreale 2016).

\section{Excluded studies}

We excluded a total of 53 studies (AMOBES 2017; Asberg 1989; Chu 2003; Di Lauro 2003; Diserens 2010; Duan 2006; Fang 2001a; Fang 2001b; Gong 2003; Gorbunov 2003; Gu 2006; Guan 2001; Hamrin 1982; Hara 2001; Huang 2001; Huang 2003; Ishida 2001; Kreisel 2005; Li 1999; Li 2003; Li 2004; Lin 2005; Liu 2001b; Liu 2003b; Liu 2004; Marshall 2011; Miskovic 2004; Pan 2004; Qian 2003; Qian 2004; Raicevic 2000; Richards 1993; Sankara Kumaran 2013; Song 2005; Sun 2002; Toyota 2001; Truscott 1974; Wang 2004; Wang 2005; Wang 2006; Wu 2012; Xi 2003; Xiao 2000; Xiao 2004; Xie 2003a; Xue 2004; Xue 2006; Xue 2008; Zeng 2004; Zhang 1998; Zhang 2001; Zhao 2003; Zheng 2004; Zheng 2004a; Zielke 2003). Many of these studies were published in Chinese language journals and required translation to English. We excluded these trials for various reasons, as detailed in the 'Characteristics of excluded studies' table. The main reasons for exclusion were late intervention, not early mobilisation intervention, timing not stated, and not randomized.

\section{Risk of bias in included studies}

The risk of bias assessments are summarized in the Characteristics of included studies, and in Figure 2 and Figure 3. 
Figure 2. Risk of bias summary: review authors' judgements about each risk of bias item for each included study

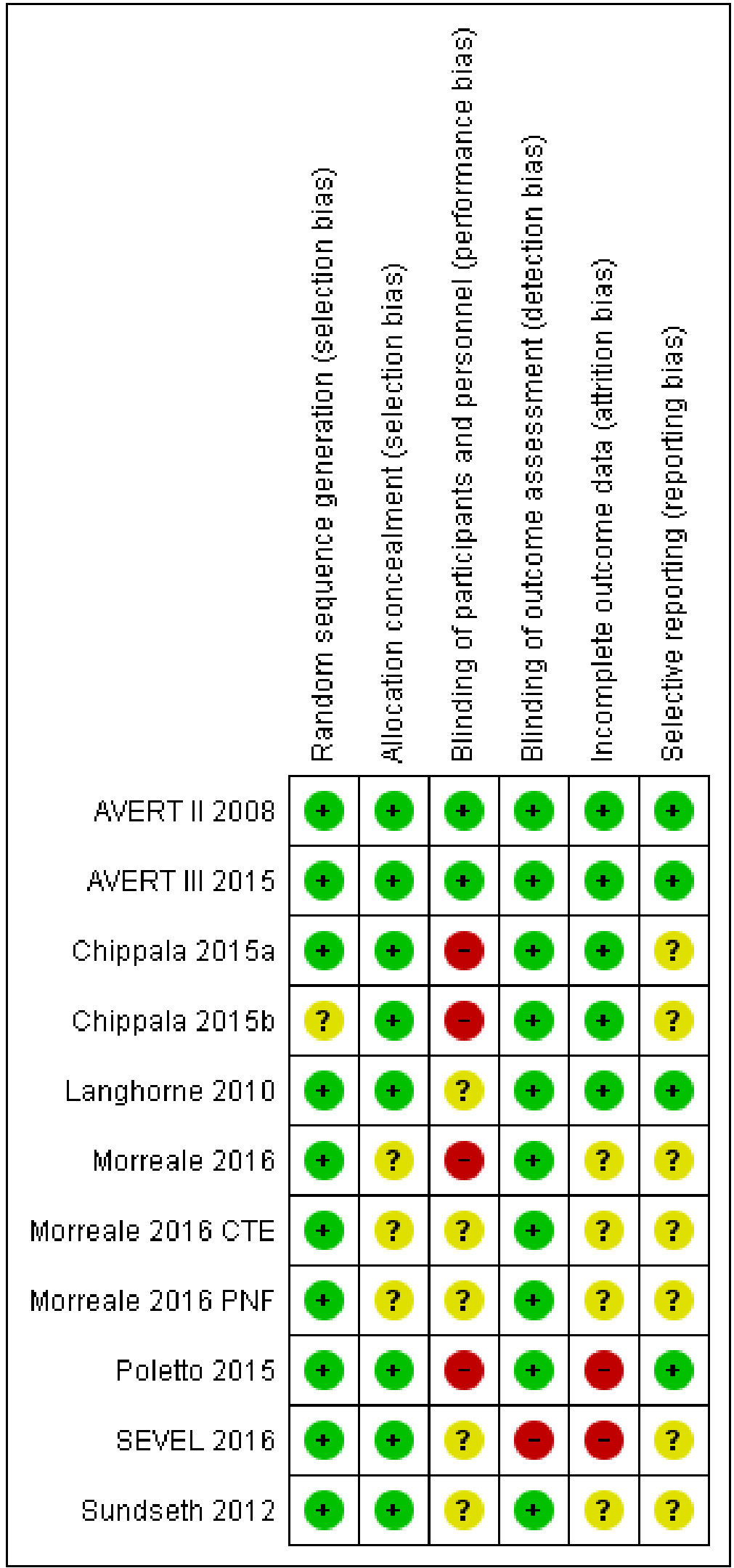


Figure 3. Risk of bias graph: review authors' judgements about each risk of bias item presented as percentages across all included studies

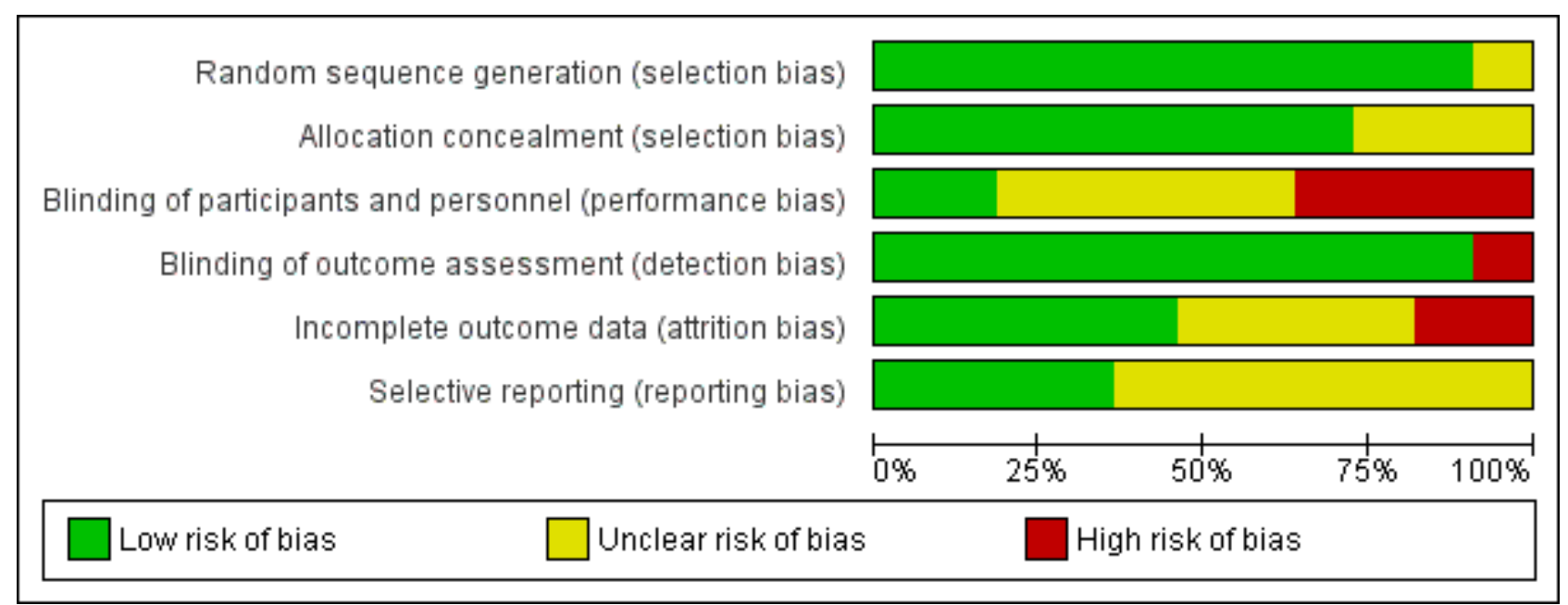

\section{Allocation}

All trials reported using a computer-generated randomization schedule. Allocation was concealed by using sequential, opaque envelopes, or a secure, centralised randomization procedure.

We assessed random sequence generation to be at low risk of bias for eight studies, and unclear for one study (Chippala 2015b).

We assessed allocation concealment to be at low risk of bias for eight studies, and unclear for one study (Morreale 2016).

\section{Blinding}

Blinding of treating therapists and patients was challenging. In three trials, the treating staff were aware of the VEM protocol, but this was masked from the rest of the staff (AVERT II 2008; AVERT III 2015; Langhorne 2010). Patients were aware they were in a trial of different protocols, but the details were not emphasised. Blinding was uncertain for the other trials. We assessed blinding to be at low risk of bias for two studies (AVERT II 2008; AVERT III 2015), at unclear risk for three studies (Langhorne 2010; SEVEL 2016; Sundseth 2012), and at high risk for four studies (Chippala 2015a; Chippala 2015b; Morreale 2016; Poletto 2015).

All but one trial used an independent (blinded) assessor for the follow up of the primary outcome (SEVEL 2016). The security of blinding was less complete for secondary outcomes, such as in hospital complications.

\section{Incomplete outcome data}

Intention-to-treat analyses were not possible with incomplete outcome data. Therefore incomplete data were described for each outcome analysis. We assessed the risk of bias to be low in five studies (AVERT II 2008; AVERT III 2015; Chippala 2015a; Chippala 2015b; Langhorne 2010); unclear in two studies (Morreale 2016; Sundseth 2012); and high in two studies (Poletto 2015; SEVEL 2016). this as dichotomous data (Morreale 2016). Data were available for 2542 of 2618 (97.1\%) participants randomized, and followed up for a median of three months. In addition, three trials also planned or conducted follow-up at one year (AVERT II 2008; AVERT III 2015; Morreale 2016).

Data were less complete for the secondary outcome measures, which were usually conducted at three months. This was probably due to missing data (rather than selective reporting) when participants had to actively complete a score. These outcomes included: ADL score, extended ADL score, subjective health status, mood score, mobility, place of residence, presence and type of complications. Complications were usually recorded during the period of hospitalisation, and were divided into: 1) complications of immobility (chest infection, urinary infection, pulmonary thromboembolism, pressure sores), plus 2) others. Specific comments were made for each outcome listed in the 'Summary of findings' table (Summary of findings for the main comparison).

We do not presently have sufficient data for the planned subdivision of other complications into neurological causes (recurrent stroke, seizure, progressing stroke - defined according to the European Progressing Stroke Study definition (Barber 2004), or a progressive deterioration in the Scandinavian Stroke Scale or National Institutes of Health Stroke Scale).

\section{Other potential sources of bias}

Several of the review authors are trialists in this area and have publications included in this review. However, we arranged trial selection decisions to avoid trialists making decisions about their own trials.

\section{Effects of interventions}

See: Summary of findings for the main comparison

\section{Selective reporting}

Reporting was largely complete for the primary outcome; nine trials included the primary outcome of death or dependence (mRS score 3 to 5) at three months post stroke, although one did not report 


\section{Primary outcome}

\section{Death or a poor outcome}

Data were available from all trials, except Morreale 2016, for the outcome of death or a poor outcome, at the end of scheduled follow-up. Within these eight trials, data were available for 2542 of 2618 participants randomized $(97.1 \%)$. The number of participants dying or having a poor outcome at the end of scheduled followup was similar in those who received VEM $(640 / 1262,50.7 \%)$ to those in the usual care group (622/1280, $48.6 \%$; odds ratio (OR) $1.08,95 \%$ confidence interval $(\mathrm{Cl}) 0.92$ to $1.26 ; \mathrm{P}=0.36$; Analysis 1.1 ; moderate-quality evidence)). There was substantial heterogeneity (68\%); reanalysis with a random-effects model did not alter the conclusions (fixed effect OR $1.08,95 \% \mathrm{Cl} 0.92$ to 1.26; random effects OR $0.77,95 \% \mathrm{Cl} 0.45$ to 1.31 ).

Analysis using outcomes collected at three-month follow-up did not alter the conclusions. It is notable that the original pre-planned analysis of the largest trial, which contributed 2104 of the total of $2618(80 \%)$ participants included, found increased odds of death or poor outcome (mRS 3 to 6 ) at three months, after adjustment for prognostic factors (adjusted OR $1.37,95 \% \mathrm{Cl} 1.11$ to $1.69 ; \mathrm{P}=0.004$; unadjusted $\mathrm{OR} 1.17,95 \% \mathrm{Cl} 0.99$ to $1.39 ; \mathrm{P}=0.06$; Analysis 2.1; AVERT III 2015).

\section{Secondary outcomes}

\section{Death}

Data were available from all trials, except Morreale 2016, for the outcome of death, at the end of scheduled followup. Within these eight trials, data were available for 2561 of 2618 participants randomized (97.8\%). There was no statistically significant difference in the number of deaths at the end of scheduled follow-up among those who received VEM (108/1274, $8.5 \%)$ compared with the control group (87/1287, 6.8\%; OR 1.27 , $95 \% \mathrm{Cl} 0.95$ to $1.70 ; \mathrm{P}=0.11$; Analysis 1.2 ; moderate-quality evidence). There was little heterogeneity $(0 \%)$. Analysis using outcomes collected at three-month follow-up did not alter the conclusions (Analysis 2.2).

\section{Death or dependence}

Data were available from all trials, except Morreale 2016, for the outcome of death or dependence (mRS 3 to 6), at the end of scheduled follow-up. Within these eight trials, data were available for 2542 of 2618 participants randomized (97.1\%). There was no clear difference in the risk of participants dying or remaining dependent in those who received VEM (640/1262, 50.7\%) compared with the control group $(622 / 1280,48.6 \%)$ at the end of scheduled follow-up (OR $1.08,95 \% \mathrm{Cl} 0.92$ to $1.26, \mathrm{P}=0.36$; Analysis 1.3; moderate-quality evidence). There was substantial heterogeneity (68\%). Re-analysis with a random-effects model did not alter the conclusions. Analysis using outcomes collected at three-month follow-up did not alter the conclusions (Analysis 2.3).

Please note that because we had access to our preferred dependence data for the primary (poor) outcome (Analysis 1.1 and Analysis 2.1), the death or dependence outcomes (Analysis 1.3 and Analysis 2.3) were identical to the primary outcome.

\section{Death or institutional care at end of scheduled follow-up}

Data were available from only three trials for the outcome of death or requiring institutional care, at the end of scheduled follow-up
(AVERT II 2008; Langhorne 2010; SEVEL 2016). Overall data were available for only 227 of 270 participants randomized (84.1\%). There was no clear difference in the risk of participants dying or requiring institutional care at the end of scheduled follow-up in those who received VEM $(21 / 112,18.8 \%)$ compared with the control group $(20 / 115,17.4 \%$; OR $1.05,95 \% \mathrm{Cl} 0.53$ to $2.07, \mathrm{P}=0.89$; Analysis 1.4). There was little heterogeneity (20\%). Analysis using outcomes collected at three-month follow-up did not alter the conclusions (Analysis 2.4).

\section{Performance in activities of daily living}

Data were available from all trials, except Chippala 2015b, for the outcome of activities of daily living (ADL) score, at the end of scheduled follow-up. All trials used the Barthel Index, so we converted scores to a standard scale of 0 to 20. Overall, data were available for 2630 of 2909 participants randomized (90.6\%). There was a higher mean ADL score for those who received VEM, compared with the control group (mean difference (MD) 1.94, 95\% $\mathrm{Cl} 0.75$ to 3.13 ; $\mathrm{P}=0.001$; Analysis 1.5 ; low-quality evidence). There was substantial heterogeneity (93\%), so we completed the analysis using a random-effects model. Cautious interpretation is also required, because the Barthel Index often has a non-normal distribution.

Most trials included terminal follow-up at three months, so many of the data are the same in both analyses. Analysis using outcomes collected at three-month follow-up resulted in a smaller observed effect size (MD 0.75, 95\% Cl 0.01 to 1.49; $\mathrm{P}=0.05$; Analysis 2.5); lowquality evidence) and a lesser degree, although still substantial, of heterogeneity $(80 \%)$, so we used a random-effects model for analysis.

\section{Performance in extended activities of daily living (community and domestic activities)}

No data were available for this outcome.

\section{Patient subjective health status and quality of life}

Only AVERT II 2008 reported this outcome in the form of the Assessment of Quality of Life scale. They found no significant difference between groups (MD $0.07,95 \% \mathrm{Cl}-0.10$ to $0.23 ; \mathrm{P}=0.42$; 1 trial, 68 participants; Analysis 1.6; very low-quality evidence).

\section{Able to walk and mobility score}

Data were available from four trials for the outcome of being able to walk at follow-up (AVERT II 2008; AVERT III 2015; Langhorne 2010; Sundseth 2012). Within these four trials, data were available for 2255 of 2272 participants randomized (99.3\%). There was no clear difference in the number of participants able to walk among those who received VEM $(831 / 1130,73.5 \%)$ compared with the control group $(827 / 1125,73.5 \%)$ at follow-up (OR $1.00,95 \% \mathrm{Cl} 0.83$ to $1.21 ; \mathrm{P}=0.99$; Analysis 1.7). There was substantial heterogeneity $(60 \%)$, but re-analysis with a random-effects model did not alter the conclusions.

Data were available from only two trials for the outcome of mobility score at the end of scheduled follow-up (AVERT II 2008; Langhorne 2010). Within these two trials, data were available for 102 of 103 participants randomized (99.0\%). There was no significant difference in the mean score between those who received VEM compared with the control group (standardized mean difference 
(SMD) $0.14,95 \% \mathrm{Cl}-0.27$ to $0.56 ; \mathrm{P}=0.50$; Analysis 1.8$)$. There was no substantial heterogeneity (8\%).

\section{Complications: any complication}

Data were available from all trials, except Chippala 2015a, Chippala 2015b, and SEVEL 2016, for the outcome of participants who reported any complication, recorded at follow-up. Within these six trials, data were available for 2778 of 2818 participants randomized $(98.6 \%)$. There was no statistically significant difference in the risk of participants developing a complication among those who received VEM $(287 / 1436,20.0 \%)$ compared with the control group $(301 / 1342,22.4 \%)$ at the end of scheduled follow-up (OR 0.88, 95\% $\mathrm{Cl} 0.73$ to $1.06 ; \mathrm{P}=0.18$; Analysis 1.9 ; low-quality evidence). There was no substantial heterogeneity (0\%).

When complications were divided into those classified as complications of immobility and other complications, there was no significant difference between groups (test for subgroup differences $P=0.23$; Analysis 1.10). We had insufficient information to analyze by severity of complication, or other types of complication.

\section{Patient mood at the end of scheduled follow-up}

Only two trials reported this outcome (AVERT II 2008; Sundseth 2012). There was no significant difference between groups (SMD $0.07,95 \% \mathrm{Cl}-0.33$ to $0.46 ; \mathrm{P}=0.74 ; 2$ trials, 100 participants; Analysis 1.11).

\section{Length of stay in acute hospital}

Data were available from all trials, except Morreale 2016, for the outcome of length of acute stay in hospital. Within these eight trials, data were available for 2551 of 2618 participants randomized (97.4\%). Seven of the eight trials reported a shorter length of stay in the VEM group. Across all trials, there was a shorter mean length of stay for the group who received VEM compared with the usual care group (MD -1.44, 95\% Cl-2.28 to -0.60; $\mathrm{P}=0.0008$; Analysis 1.12; lowquality evidence). There was no substantial heterogeneity (26\%), but we completed the analysis using a random-effects model, because of the variable definitions of length of stay. This result was largely driven by two small trials, with narrow standard deviation values (Chippala 2015a; Chippala 2015b). Cautious interpretation is also required because the length of stay often has a non-normal distribution.

\section{Sensitivity analysis}

Trial quality: none of the outcome conclusions were altered if we restricted analysis to trials with the highest methodological quality (based on method of randomization, allocation concealment, completeness of follow-up, and blinding of final assessment), or information about the amount of mobilisation.

Intervention features: sensitivity analyses restricted to trials with an earlier time-to-first mobilisation (mean time-to-first mobilisation of less than 24 hours) showed a similar primary outcome to the main analysis (OR 1.10, 95\% Cl 0.93 to 1.29; $\mathrm{P}=0.27$; $\left.\mathrm{I}^{2}=78 \%\right)$, and an odds of death of $1.35(95 \% \mathrm{Cl} 0.99$ to $1.83 ; \mathrm{P}=0.06$; $I^{2}=25 \%$; AVERT II 2008; AVERT III 2015; Chippala 2015a; Chippala 2015b; Sundseth 2012). Analyses restricted to the trials that clearly recorded a more prolonged out-of-bed activity, showed a similar primary outcome (OR $1.14,95 \% \mathrm{Cl} 0.96$ to $1.35 ; \mathrm{P}=0.13 ; \mathrm{I}^{2}=28 \%$ ), and odds of death (OR $1.27,95 \% \mathrm{Cl} 0.93$ to $1.73 ; \mathrm{P}=0.13 ; \mathrm{I}^{2}=0 \%$ ) to the main analysis (AVERT II 2008; AVERT III 2015; Langhorne 2010; Poletto 2015).

\section{Network meta-analysis (NMA)}

This review included trials that aimed to compare the effect of reducing time-to-first mobilisation (TTFM), with or without an increase in the amount or frequency of mobilisation activities, with usual care (where TTFM started later). However, it was clear that within this definition, the included trials would comprise a range of different comparisons. Therefore, we conducted an exploratory, post-hoc, network meta-analysis (NMA) to explore, where possible, the impact of treatment characteristics (TTFM and amount of mobilisation activity). NMA analysis by the amount of mobilisation activity was not possible, because this information was not consistently reported (Table 1). However, we could categorise information on TTFM into five major TTFM groups, with limited overlap.

Table 2 shows the categories of TTFM, with early mobilisation TTFM in the columns, and usual care TTFM in the rows. The lower left section indicates the trials (participants) contributing to each direct comparison of TTFM. Figure 4 shows the same comparisons in the form of a network plot. 
Figure 4. Network plot of all trials. Each point shows the time-to-first mobilisation (TTFM) classifications. The lines show the number of trials directly comparing each TTFM category.

\section{Network plot of all studies}

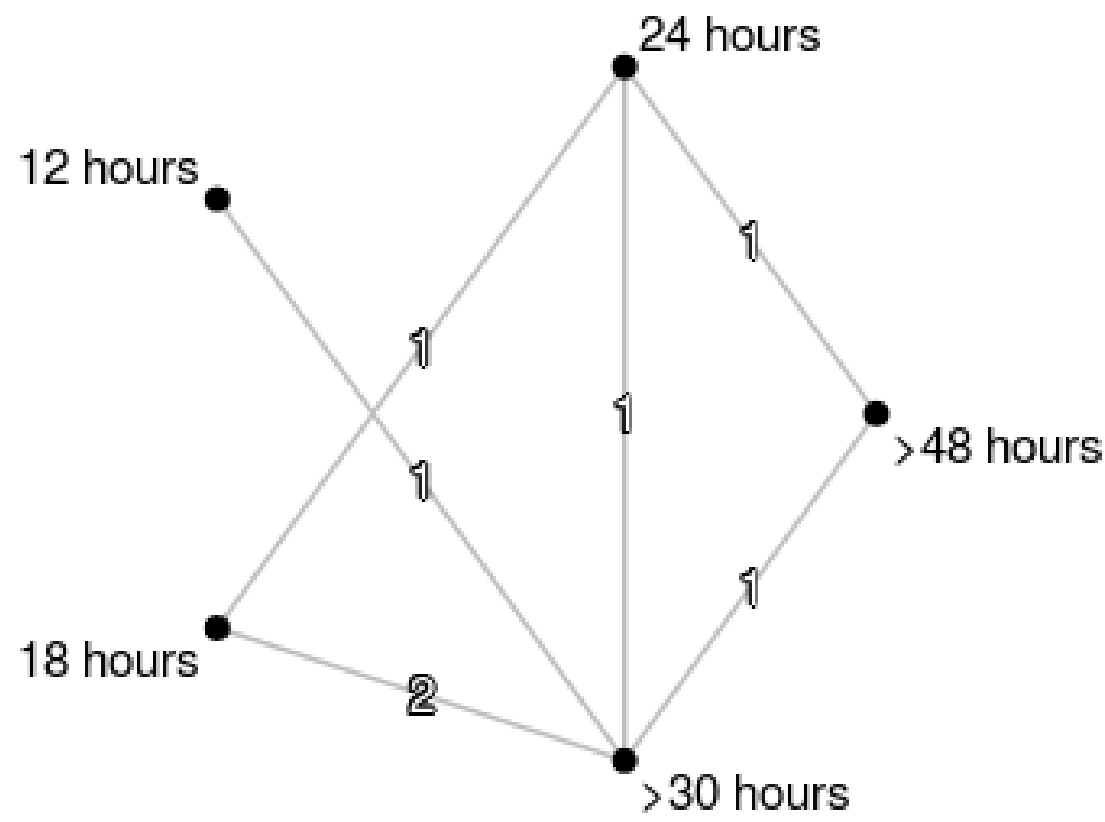

We believe that the transitivity (or similarity) assumption was met, as all the included trials recruited people with acute stroke, within 48 hours of symptom onset, within a stroke unit (or similar) setting. The IQR distribution for average age (65 to 73 years), gender ( $45 \%$ to $57 \%$ male), and stroke severity (44\% to $68 \%$ moderate or severe) were broadly comparable (Table 1 ).

We evaluated the consistency assumption statistically, by comparing the difference between the direct and the indirect estimate for each loop of evidence. We examined for any inconsistency (i.e. important differences in numerical results between direct, indirect, and network results), and we presented OR estimates for each of the three comparisons.
We showed the inconsistency tables for the NMA for the analyses of poor outcome (Table 3), and death (Table 4). These tables show the results of direct and indirect comparisons, plus the NMA results. There were no statistically significant differences between any of the direct and indirect comparisons, but the confidence intervals were wide (Table 5).

The NMA used the 24-hour TTFM group as the comparator, as this was clinically relevant and incorporated the largest single trial (AVERT III 2015). Figure 5 shows the NMA result for the poor outcome (death or dependency) at three months. The lowest odds of poor outcome was at 24 hours, although confidence intervals were very wide. A similar pattern was seen for the outcome of death at three months (Figure 6), but again with very wide confidence intervals (Table 5). 
Figure 5. Network meta-analysis plot for poor outcome (death or dependency at 3 months). The treatment column shows the time-to-first mobilisation (TTFM) categories. The results are the odds ratio ( $95 \%$ confidence interval) for the odds of a poor outcome with TTFM of 24 hours as the reference $(O R=1.0)$.

\section{Results for all studies}

\begin{tabular}{|c|c|c|c|c|}
\hline Treatment & Random Ef & fects Model & OR & $95 \%-\mathrm{Cl}$ \\
\hline 12 hours & & & 6.61 & {$[1.36 ; 32.09$} \\
\hline 18 hours & & + & 1.07 & {$[0.53 ; 2.19]$} \\
\hline 24 hours & & & 1.00 & \\
\hline$>30$ hours & & $\longrightarrow$ & 2.74 & {$[1.18 ; 6.37$} \\
\hline$>48$ hours & & + & 1.29 & {$[0.50 ; 3.37]$} \\
\hline & 1 & 1 & & \\
\hline & 0.51 & 10 & & \\
\hline
\end{tabular}


Figure 6. Network meta-analysis plot for death at 3 months. The treatment column shows the time-to-first mobilisation (TTFM) categories. The results are the odds ratio ( $95 \%$ confidence interval) for the odds of death with TTFM of 24 hours as the reference $(O R=1.0)$.

\section{Results for all studies}

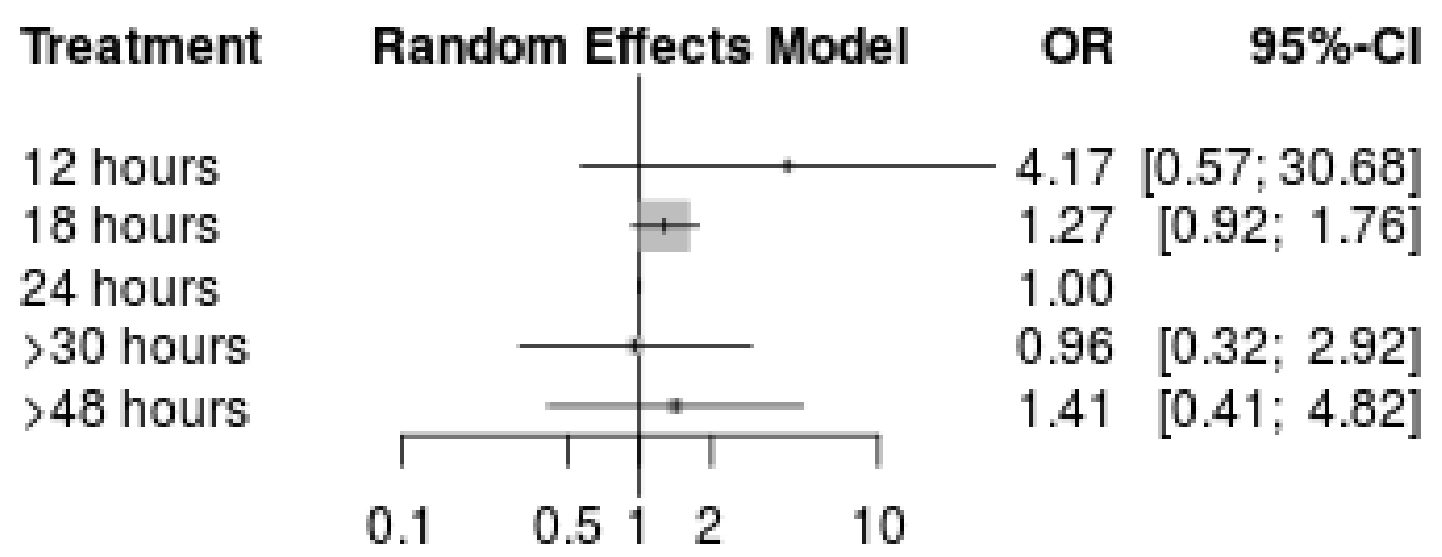

Finally, we carried out a Rank analysis, which orders treatments according to their relative effectiveness; the first ranked treatment is most likely to be the most effective treatment, compared with the other treatments in the network. This analysis rated the 24hour TTFM group to be the one most likely to be associated with efficacy ( $P=0.81$ for poor outcome), and safety ( $P=0.76$ for death by three months), indicating that 24-hour TTFM was the optimal option, although our confidence was limited by the substantial imprecision.

\section{DISCUSSION}

This systematic review aimed to assess the benefits or harms of very early mobilisation (VEM) compared with conventional practice (usual care). We conducted a comprehensive search of citation and clinical trial registries, and contacted researchers in the field to identify unpublished studies. We identified 13 eligible RCTs, nine (2958 participants) of which we included: three from China are currently underway or not yet published, and one is in the planning stage (AVERT-DOSE 2017). 


\section{Summary of main results}

The included trials incorporated different approaches to VEM, but all included an earlier onset of out-of-bed mobilisation, and several reported providing a higher intensity (minutes per day) of mobilisation activity. The primary outcome (death or poor outcome) was not significantly altered by the mobilisation strategy. Neither were the other major binary outcomes: death, death or dependency, death or requiring institutional care, presence of complications, or ability to walk. We assessed the evidence for these outcomes as being of moderate quality. There was an indication that VEM may result in a higher ADL score among survivors, and a slightly shorter length of hospital stay. However, these conclusions were based on less reliable data that may have non-normal distributions, and are more prone to bias (low-quality evidence). Sensitivity analyses raised the possibility that trials with an earlier onset of VEM may have had a higher risk of death at the end of follow-up.

The review was dominated by one large, multicentred trial, involving 2104 participants from six countries (AVERT III 2015). The results of AVERT III 2015 suggested a hazard with VEM (adjusted odds ratio (OR) for death or dependency at three months was $1.37,95 \%$ confidence interval $(\mathrm{Cl}) 1.11$ to $1.69 ; \mathrm{P}=0.004)$. Prespecified dose-response analyses suggested that better outcomes were associated with more frequent, and less prolonged episodes of mobilisation (AVERT III 2015).

\section{Overall completeness and applicability of evidence}

Clearly, there is considerable research interest in this field; we identified a number of recent trials. Despite this significant body of potentially relevant research, very few studies met the inclusion criteria. One of the major problems we identified during our search for relevant research, was that the term 'early rehabilitation' was used to define interventions spanning a wide time interval. For example, some authors used the term 'early' to describe an intervention that commenced within 48 hours post stroke (Xue 2004), while other authors described interventions commencing within three months of stroke as 'early' interventions (Li 2002). It is likely that what clinicians and researchers consider as early rehabilitation is strongly dependent on when rehabilitation usually commences within their healthcare system, and that this varies considerably between countries.

The most common reason we excluded trials from this review was that the mobilisation interventions in the experimental group commenced more than 48 hours after stroke symptom onset. Also, a few studies failed to detail the time from stroke onset, instead noting time from admission (e.g. Asberg 1989; Gao 2001). Given that it can take patients hours or even days to reach hospital following a stroke, these studies failed to provide a precise time point from stroke onset to commencing intervention, and consequently, we could not include the trials. Other studies provided insufficient information about the timing of the intervention to allow confirmation that the trial met the inclusion criteria. We attempted to contact study authors, and we are awaiting responses. Poor definition of the intervention content and dose was also a problem in this review. It was not uncommon to find interventions only broadly defined, with limited information about how much mobilisation was delivered by whom, how often, and over what time frame (days or weeks). Once again, we attempted to contact the study authors to seek further information.

Despite the uncertainties within the research field, our included trials, in particular AVERT III 2015, appeared to have included a representative patient population of those who are receiving cared in routine stroke unit settings. These trials generally took a pragmatic approach, and recruited a reasonably representative population in terms of age, sex, stroke type, and stroke severity. The data completeness for the key binary outcomes was generally good, but less secure for the continuous outcomes.

We planned the analysis of treatment characteristics in the expectation that within our broad comparison of early versus later onset mobilisation, the included trials would comprise a range of treatment comparisons. Therefore, we included an exploratory post-hoc network meta-analysis (NMA) to explore the impact of treatment characteristics (time-to-first mobilisation, and amount of mobilisation activity). We were unable to conduct NMA analysis by the amount of mobilisation activity because this information was not consistently reported between trials. However, we could categorise information on time-to-first mobilisation (TTFM) into five major TTFM groups with limited overlap. This analysis suggested that for both death and poor outcome (death or dependency) at three months, the optimal TTFM was about 24 hours. However, considerable caution is required in interpreting this result. First, this was an exploratory post-hoc analysis. Second, the TTFM categories were not absolutely discrete groups; we imposed the classification to fit the available trials. Third, the trials differed in type and quantity of mobilisation activity as well as TTFM. As a result, the confidence intervals were wide, and we graded the quality of the evidence as low. Therefore, we could conclude that further trials were warranted, but the limited information available at present did not indicate that any TTFM was superior to 24 hours (IQR 22 to 29 ).

\section{Quality of the evidence}

The nine trials included in the review showed a low risk of selection bias and were largely secure against detection bias (Figure 2). The most challenging aspect was the blinding of staff and participants. For some rehabilitation interventions, such as VEM, it may not be possible to have a truly double-blinded study; however, several trials attempted to mitigate such potential sources of bias (AVERT II 2008; AVERT III 2015; Langhorne 2010; Morreale 2016). First, participants were informed that they could be randomized to one of two styles of rehabilitation (AVERT II 2008), or treatment protocols (AVERT III 2015; Langhorne 2010), but the details of these protocols were not usually explained in detail. Second, the intervention was not made available to personnel beyond the treating staff, and efforts were made to avoid contamination (e.g. by providing treatment behind curtains (AVERT II 2008; AVERT III 2015; Langhorne 2010; Morreale 2016)).

\section{Potential biases in the review process}

Several of the review authors (JB, PL, JC, AS) were trialists in at least one of the included trials. However, we ensured that trial selection decisions were allocated in a manner that avoiding trialists making decisions about their own trials.

Our review identified a substantial number of trials from China that addressed the topic of early rehabilitation following stroke. We 
understand that current usual practice in many places in China, is to provide little or no organised rehabilitation to people following stroke. Therefore, it was likely that these trials, many of which were undertaken over the past 10 years, reflected an increased interest in the provision of rehabilitation to people with stroke. Unfortunately, none of Chinese trials we identified in this review met the inclusion criteria, because they provided either a complete multidisciplinary rehabilitation program or package, which may or may not have included a mobilisation component, and compared this with no rehabilitation (e.g. Xue 2004; Liu 2010e), or did not provide details of the time of commencement of mobilisation (e.g. Zhang 1998). In view of this rapidly growing body of Chinese research and the difficulties associated with acquisition and translation of Chinese research, it would be beneficial to form partnerships with Chinese researchers on the topic of early rehabilitation (Zhang 2014).

The analyses of continuous outcomes reported in this review were all subject to potential bias, and we rated them as providing lowquality evidence (Summary of findings for the main comparison). Although the main measure of activities of daily living (the Barthel $\mathrm{ADL}$ index) is a 20-point ordinal scale, its characteristics in people with stroke approximate to that of a continuous scale, and analysis using mean and standard deviation is usually justifiable (Song 2006). However, we downgraded the ADL data, because there was a high level of statistical heterogeneity, and a substantial rate of missing data (for instance, participants who died were not included). Analysis of length of stay was confounded by the variable definitions of length of stay, and the likelihood that these data may be non-normally distributed. For both outcomes, re-analysis of individual patient data would provide a more reliable estimate of effect.

The network meta-analysis was exploratory in nature and had a number of uncertainties. Therefore, we judged the evidence for these conclusions to be low quality (Table 5).

\section{Agreements and disagreements with other studies or reviews}

This review update appeared to add substantive information to this field. Recent reviews published in this area identified fewer new RCTs, and concluded that the benefits of commencing physical rehabilitation within 24 hours of stroke were unclear. Lynch 2014 identified three new RCTs, and Bernhardt 2015 identified four RCTs, and concluded that the evidence was inconclusive. Whaley 2016 included two trials, and concluded that evidence supported a rested approach to care within the first 24 hours of hospitalisation. Finally, a recent review with different inclusion criteria concluded that there were no beneficial effects from VEM (Xu 2017).

\section{AUTHORS' CONCLUSIONS}

\section{Implications for practice}

The approach to very early mobilisation (VEM) described in the trials we reviewed always featured an earlier onset of out-ofbed mobilisation activities, and often reported providing a higher intensity (time per day) of mobilisation activity. Overall, there was no significant impact of VEM on the main clinical outcomes (death, dependency, institutional care, presence of complications, ability to walk), although the largest single trial, AVERT III 2015, found a significant increase in death or dependency. We based our suggestions that VEM may result in a higher ADL score among survivors and a slightly shorter length of hospital stay on less reliable data that were more prone to bias.

We believe that the evidence supported a cautious approach to active mobilisation within 24 hours of stroke onset because the single largest trial (AVERT III 2015), and a sensitivity analysis of trials recruiting within 24 hours, raised the possibility that VEM commencing within 24 hours may carry some increased hazard. In addition, low-quality evidence from an exploratory network metaanalysis indicated that mobilisation at around 24 hours may be associated with the best outcome.

\section{Implications for research}

Larger, high-quality trials of VEM are needed to clarify the relative harms and benefits of different mobilisation strategies. In particular, these studies need to clearly define the optimal timing of commencement, frequency, and duration of mobilisation interventions. Timing should be provided from time of stroke symptom onset, not admission. Researchers must ensure publications fulfil CONSORT guidelines, with adequate description of the experimental and control interventions (Moher 2001).

We recommend that researchers and clinicians move to using a common terminology, as outlined in recent recommendations to improve the development and reporting of rehabilitation trials (Bernhardt 2017). We have proposed the term 'very early' rehabilitation to indicate interventions commencing within two days, and 'early' as those commencing three to seven days following stroke onset (Bernhardt 2007). In this time-critical field of research, it is also important that researchers clearly define when interventions commenced (hours), and that the time is estimated from the start of stroke symptom onset. Interventions should be described in a standard manner, such as with the TIDieR framework (Hoffman 2014). In the interim, an individual patient data metaanalysis of the existing trials may help explore some of the current uncertainties.

\section{ACKNOWLEDGEMENTS}

For this update of the review, we acknowledge the support of: Dr Wenwen Zhang (The Florey Institute) for help getting papers, interpreting and translating the Chinese research; Ms Ye Liu and Dr Jue Wang (The Florey Institute) for translating abstracts; Ms Rosemary Morrison for cross-checking studies identified; $\mathrm{Mr}$ Joshua Cheyne (Cochrane Stroke Group information Specialist); Hazel Fraser and the Cochrane Stroke Group; University of Melbourne with full text articles; Dr Karin Diserins and Patrik Michel (Department of Clinical Neurosciences, Centre Hospitalier Universitaire and University of Lausanne, Switzerland), and Antje Sundseth (Akershus Hospital, Norway) for trial information. We are grateful to Jonathan M Fuchs for his consumer review.

For the original version of the review, we acknowledge the following for their support of this review: Dr Jue Wang (The Florey Institute) and DrWenwen Zhang (The Florey Institute) for help getting papers, interpreting, and translating the Chinese research; the Austin Health Interpreting and Transcultural Services (Austin Health, Melbourne, Australia) for help with Chinese language interpreting; Ms Li Chun Quang (The Florey Institute) and Ms Kim Ong (The Florey Institute) with technology support; Brenda Thomas (Cochrane Stroke Group Trials Search Co-ordinator) and Lynsey Smyth (STEP program, University of Glasgow) with searches; Hazel Fraser and 
the Cochrane Stroke Group, Ms Nancy Guan with Chinese Academic Journals, the Austin Health Sciences Library staff (Austin Health, Melbourne, Australia), Ms Bick-har Yeung (East Asian Library, University of Melbourne), and the University of Melbourne with full-text articles; Ms Dianna Sorbello (The Florey Institute) and Ms Mingming Zhang (Chinese Cochrane Center) for general assistance; Dr Dong Junli (Department of Neurology, Yunyang Medical College, Hubei Province, China), Dr William J Peek (International Society of Physical and Rehabilitation Medicine), Mr Werner Van Cleemputte (ISPRM) and Prof Peter Langhorne (Academic Section of Geriatric Medicine, Royal Infirmary, Glasgow, UK) for manuscripts; Dr
Stefan Kreisel (Dept of Neurology, University of Heidelberg, Mannheim, Germany), Dr Andrea Di Lauro (U. O. Neurologia, Azienda Ospedaliera S. Sebastiano, Via Palasciano, Caserta, Italy), Prof Valerie Pomeroy (Section of Geriatric Medicine, Division of Clinical Developmental Sciences, St George's University of London, UK), Dr Stefano Paolucci (Fondazione S. Lucia - IRCCS, Rome, Italy) and Dr Michal Katz-Leurer (Department of Physiotherapy, Tel Aviv University, Ramat Aviv, Israel) for further information on their studies; and Dr Di Lauro and Prof Lorraine Smith (Nursing \& Health Care, Faculty of Medicine, University of Glasgow, UK) for expert opinion on identifying unpublished studies. 


\section{R E F E R E N C E S}

\section{References to studies included in this review}

AVERT II 2008 \{published and unpublished data\}

Bernhardt J. Meeting the challenges of developing and conducting a multi-centre randomised controlled trial of very early rehabilitation (AVERT). Internal Medicine Journal 2007;37 Suppl 1:A2. [Ref 12276]

Bernhardt J, Chan J, Nicola I, Collier JM. Little therapy, little physical activity: rehabilitation within the first 14 days of organized stroke unit care. Journal of Rehabilitation Medicine 2007;39:43-8. [Ref 12551]

Bernhardt J, Cumming T, Ha J. Response to letter by Freeman et al regarding article, "Very early mobilization after stroke fasttracks return to walking: further results from the phase II AVERT randomized controlled trial". Stroke 2011;42:e585. [Ref 18479]

Bernhardt J, Cumming T, Thrift A, Collier J, Leonid C, Dewey H, et al. Very early mobilisation after stroke fast tracks returning to walk: further results from a phase II randomised controlled trial (AVERT). International Journal of Stroke 2011;6 Suppl 1:27. [Abst O84; [Ref 17957] ]

Bernhardt J, Cumming TB, Thrift AG, Collier JM, Churilov L, Dewey $\mathrm{H}$, et al. Very early mobilisation after stroke fast tracks returning to walk: further results from a phase II randomised controlled trial (AVERT). International Journal of Stroke 2010;5 Suppl 2:59. [Abst FC70004; [Ref 16946] ]

Bernhardt J, Dewey H, Collier J, Lindley R, Thrift A, Donnan G. A Very Early Rehabilitation Trial (AVERT): phase II safety and feasibility results. Internal Medicine Journal 2007;37 Suppl 1:A9. [Ref 12266]

Bernhardt J, Dewey H, Collier J, Thrift A, Donnan G. A pilot randomized controlled trial to evaluate the safety and feasibility of very early mobilization in acute stroke units (AVERT). Physiotherapy 2007;93 Suppl 1:S501. [Abst 3258; Ref 16562]

Bernhardt J, Dewey H, Collier J, Thrift A, Lindley R, Moodie M, et al. A Very Early Rehabilitation Trial (AVERT). International Journal of Stroke 2006;1:169-71. [Ref 11165]

Bernhardt J, Dewey H, Collier J, Thrift A, Sharpley T, Donnan G. A Very Early Rehabilitation Trial (AVERT) phase II safety and feasibility results. Stroke 2007;38(2):473. [Abst 86; Ref 11479]

Bernhardt J, Dewey H, Collier J, Thrift A, Sharpley T, Donnan G. Safety and feasibility results of a very early rehabilitation trial (AVERT): phase II. Cerebrovascular Diseases 2007;23 Suppl 2:6. [Abst 6; Ref 12127]

Bernhardt J, Dewey H, Thrift A, Collier J, Aldridge V, Donnan G. A Very Early Rehabilitation Trial (AVERT) - phase II pilot study. Internal Medicine Journal 2005;35 Suppl 2:A9. [Ref 12119]

Bernhardt J, Dewey H, Thrift A, Collier J, Aldridge V, Donnan G. A Very Early Rehabilitation Trial (AVERT) - phase II pilot study. Journal of Clinical Neuroscience 2004;11 Suppl 1:S30. [Abst POS1E 18; Ref 9679]
* Bernhardt J, Dewey H, Thrift A, Collier J, Donnan G. A Very Early Rehabilitation Trial for Stroke (AVERT): Phase II safety and feasibility. Stroke 2008;39:390-6.

Bernhardt J, Dewey H, Thrift A, Collier J, Donnan G. A Very Early Rehabilitation Trial for stroke (AVERT): phase II safety and feasibility. Stroke 2008;39:390-6. [Ref 12991]

Bernhardt J, Dewey HM, Collier JM, Thrift AG, Donnan GA. Feasibility and safety of a multicentre randomised controlled trial of very early rehabilitation (AVERT): Phase II. Cerebrovascular Diseases 2006;21 Suppl 4:135. [Abst 10; Ref 10623]

Collier J, Bernhardt J. Does acute stroke unit care change during a rehabilitation clinical trial (AVERT Phase II)?. Cerebrovascular Diseases 2007;23 Suppl 2:96. [Abst 11; Ref 12115]

Collier JC, Bernhardt J. The physiotherapy pill: can clinicians provide a specified dose in a clinical trial?. Australian Journal of Physiotherapy 2008;54 1 Suppl:S12. [Ref 16287]

Collier JM, Bernhardt J. Clinical trial data collection using a personal digital assistant. Internal Medicine Journal 2005;35 Suppl 2:A17. [Ref 12121]

Collier JM, Cumming TB, Thrift AG, Bernhardt J. The effect of very early mobilisation on mood after stroke. Cerebrovascular Diseases 2008;25 Suppl 2:30-1. [Abst 6; Ref 13544]

Cumming TB, Collier J, Thrift AG, Bernhardt J. The effect of very early mobilization after stroke on psychological well-being. Journal of Rehabilitation Medicine 2008;40:609-14. [Ref 17240]

Cumming TB, Plummer-D'Amato P, Linden T, Bernhardt J. Hemispatial neglect and rehabilitation in acute stroke. Archives of Physical Medicine and Rehabilitation 2009;90:1931-6. [Ref 18464]

Cumming TB, Thrift AG, Collier J, Bernhardt J. The effect of very early mobilisation on mood after stroke. Internal Medicine Journal 2007;37 Suppl 4:A105. [Ref 17347]

Cumming TB, Thrift AG, Collier JM, Churilov L, Dewey HM, Donnan GA, et al. Very early mobilization after stroke fasttracks return to walking. Further results from the phase II AVERT randomized controlled trial. Stroke 2011;42:153-8. [Ref 16927]

Freeman WD, Chavez OS, Meschia J. Letter regarding article: Very early mobilization after stroke fast-tracks return to walking: further results from the phase II AVERT randomized controlled trial. Stroke 2011; Vol. 42:e375. [Ref 17586]

Quah D, Collier JC, Purvis T, Bernhardt J. Keeping patients in clinical trials: how was it done?. Australian Journal of Physiotherapy 2008;54 Suppl 1:S7. [Ref 16286]

Sorbello D, Bernhardt J. Sorbello D, Bernhardt J. The effect of very early mobilisation on the number and severity of complications experienced by stroke patients. Internal Medicine Journal 2007;37 Suppl 4:A106. [Ref 17348] 
Sorbello D, Dewey HM, Churilov L, Thrift AG, Collier JM, Donnan $\mathrm{G}$, et al. Very early mobilisation and complications in the first 3 months after stroke: further results from phase II of $A$ Very Early Rehabilitation Trial (AVERT). Cerebrovascular Diseases 2009;28:378-83. [Ref 16238]

Tay-Teo K, Moodie M, Bernhardt J, Thrift A, Collier J, Donnan G, et al. Economic evaluation alongside a phase II multi-centre randomised controlled trial of very early rehabilitation after stroke (AVERT). Cerebrovascular Diseases 2007;23 Suppl 2:30. [Abst 3; Ref 12129]

Tay-Teo K, Moodie M, Bernhardt J, Thrift A, Collier J, Donnan G, et al. Economic evaluation alongside a phase II, multi-centre, randomised controlled trial of very early rehabilitation after stroke (AVERT). Internal Medicine Journal 2007;37 Suppl 4:A105. [Ref 17345]

Tay-teo K, Moodie M, Bernhardt J, Thrift AG, Collier J, Donnan G, et al. Economic evaluation alongside a phase II, multi-centre, randomised controlled trial of very early rehabilitation after stroke (AVERT). Cerebrovascular Diseases 2008;26:475-81. [Ref 14455]

Tyedin K, Cumming TB, Bernhardt J. Quality of life: an important outcome measure in a trial of very early mobilisation after stroke. Disability and Rehabilitation 2010;32(11):875-84 [Ref 19272]

van Wijk R, Cumming T, Churilov L, Donnan G, Bernhardt J. An early mobilization protocol successfully delivers more and earlier therapy to acute stroke patients: further results from phase II of AVERT. Neurorehabilitation and Neural Repair 2012;26(1):20-6. [Ref 18488]

van Wijk RM, Churilov L, Bernhardt J. Intervention protocol increases frequency and amount of early mobilisation of acute stroke patients: results from a phase II RCT (AVERT). Cerebrovascular Diseases 2009;27 Suppl 6:25. [Abst 7; Ref 14804]

\section{AVERT III 2015 \{published data only\}}

ACTRN12606000185561. AVERT III. www.anzctr.org.au/ trial_view.aspx?ID=1266 (first received 7 May 2006). [ACTRN12606000185561]

* AVERT Trial Collaboration group. Efficacy and safety of very early mobilisation within $24 \mathrm{~h}$ of stroke onset (AVERT): a randomised controlled trial. Lancet 2015;386:46-55.

AVERT Trialists' Collaboration. A very early rehabilitation trial (AVERT): ongoing phase III trial efficacy \& cost effectiveness study. International Journal of Stroke 2011;6 Suppl 1:47-8. [Abst P51; Ref 17961]

Ancliffe A. Maximising recruitment to a stroke clinical trial at Royal Perth Hospital: 1000 patients screened. International Journal of Stroke 2009;4 Suppl 1:27. [Abst B28; Ref 15007]

Ancliffe J. Growing nursing and allied health rehabilitation clinical trialists: the AVERT Perth experience. Internal Medicine Journal 2007;37 Suppl 4:A116. [Abst 30; Ref 17351]
Bernhardt J. A Very Early Rehabilitation Trial (AVERT): ongoing phase III trial of efficacy and cost effectiveness of early mobilisation. International Journal of Stroke 2010;5 Suppl 1:45. [Abst P50; Ref 16662]

Bernhardt J. A Very Early Rehabilitation Trial (AVERT): ongoing phase III trial of efficacy and cost effectiveness of early mobility training after stroke. International Stroke Conference 2011. 2011 February 8-11; Los Angeles, USA. 2011. [Abst CTP29; Ref 17010]

Bernhardt J. AVERT: ongoing phase III, international trial of very early rehabilitation after stroke. International Journal of Stroke 2012;7 Suppl 1:60. [Ref 19992]

Bernhardt J. Meeting the challenges of developing and conducting a multi-centre randomised controlled trial of very early rehabilitation (AVERT). Internal Medicine Journal 2007;37 Suppl 1:A2. [Ref 12276]

Bernhardt J. Thirty hospitals and counting: developing a multicentre, international, stroke rehabilitation trial (AVERT). Australian Journal of Physiotherapy 2009;55(4 Suppl):4. [Ref 16289]

Bernhardt J, Churilov L, Dewey H, Lindley RI, Moodie M, Collier J, the AVERT Collaborators. Statistical analysis plan (SAP) for A Very Early Rehabilitation Trial (AVERT): an international trial to determine the efficacy and safety of commencing out of bed standing and walking training (very early mobilization) within $24 \mathrm{~h}$ of stroke onset vs. usual stroke unit care. International Journal of Stroke 2015;10:23-4.

Bernhardt J, Churilov L, Ellery F, Collier J, Chamberlain J, Langhorne P, AVERT Trialists' Collaboration group. Pre-specified dose response analysis for a very early rehabilitation trial (AVERT). Neurology (accessed prior to 9 August 2018):10.1212/ WNL.0000000000002459. [Bernhardt 2016]

Bernhardt J, Collier J, Lindley R, Dewey H, Thrift A, Langhorne P, et al. Stroke patients treated with alteplase in a very early rehabilitation trial (AVERT phase III). International Journal of Stroke 2010;5 Suppl 2:83. [Abst PO10038; Ref 16950]

Bernhardt J, Collier J, Thrift A, Dewey H, Lindley R, Donnan G, et al. Safety in the first 700 patients in A Very Early Rehabilitation Trial (AVERT). Stroke 2011;42(3):e77. [Abst 116; Ref 17621]

Bernhardt J, Cumming T, AVERT Trialists' Collaboration. [Ongoing international trial of very early stroke rehabilitation (AVERT)]. International Stroke Conference 2013. 2013 February 6-8; Honolulu, Hawaii, USA. 2013. [Abst CT P16; Ref 20158]

Bernhardt J, Dewey H, Collier J, Thrift A, Lindley R, Moodie M, et al. A Very Early Rehabilitation Trial (AVERT). International Journal of Stroke 2006;1:169-71.

Bernhardt J, Dewey H, Collier J, Thrift A, Lindley R, Moodie M, et al. A Very Early Rehabilitation Trial (AVERT). International Journal of Stroke 2006;1:169-71. [Ref 11165]

Bernhardt J, Dewey H, Collier J, Thrift A, Lindley R, Moodie M, et al. A Very Early Rehabilitation Trial (AVERT): ongoing phase III trial efficacy \& cost effectiveness study. Proceedings of the 18th 
European Stroke Conference 2009. 26-29 May 2009; Stockholm, Sweden. 2009:(Abst. OAID 45). [Ref 14755]

Bernhardt J, Dewey H, Collier J, Thrift A, Lindley R, Moodie M, et al. A Very Early Rehabilitation Trial (AVERT): ongoing phase III trial testing efficacy \& cost effectiveness of very early mobilisation after stroke. International Journal of Stroke 2008;3 Suppl 1:257 (Abst.PO01-601). [Ref 13925]

Bernhardt J, Dewey H, Collier J, Thrift A, Lindley R, Moodie M, et al. A Very Early Rehabilitation Trial (AVERT): ongoing phase III trial testing efficacy \& cost effectiveness of very early mobilisation after stroke. Proceedings of the 17th European Stroke Conference. 13-16 May 2008; Nice, France. 2008:(Abst. 71). [Ref 13313]

Bernhardt J, Dewey H, Collier J, Thrift A, Lindley R, Moodie M, et al. A Very Early Rehabilitation Trial (AVERT): ongoing phase III trial testing efficacy and cost effectiveness of very early mobilisation after stroke. Internal Medicine Journal 2008;38 Suppl 4:A110 (Abst.Poster 92). [Ref 13640]

Bernhardt J, Dewey H, Collier J, Thrift A, Moodie M, Lindley R, et al. A Very Early Rehabilitation Trial (AVERT): ongoing phase III trial testing efficacy \& cost effectiveness of very early mobilisation after stroke. [Abstract]. Proceedings of the International Stroke Conference 2008. 20-22 February 2008; New Orleans, USA. American Stroke Association. 2008:(Abst. CT P22). [Ref 12959]

Bernhardt J, Dewey H, Collier J, Thrift, Lindley R, Moodie M, et al. A very early rehabilitation trial (AVERT): On going phase III trial efficacy \& cost effectiveness study. International Journal of Stroke 2010;5 Suppl 2:189. [Abst PO10408; Ref 16958]

Bernhardt J, Dewey H, Collier JM, Thrift A, Lindley R, Moodie M, et al. Safety in the first 170 patients of A Very Early Rehabilitation Trial (AVERT Phase III). Cerebrovascular Diseases 2008;25 Suppl 2:45. [Abst 1; Ref 13551]

Bernhardt J, Ellery F, Borschmann K. The tribulations and the truth about recruiting sites to a large international clinical trial. Stroke 2011;42(3):e77. [Abst 115; Ref 17620]

Bernhardt J, Raffelt A, Churilov L, Lindley RI, Speare S, Ancliffe J, AVERT Trialists' Collaboration. Exploring threats to generalisability in a large international rehabilitation trial (AVERT). BMJ Open 2015;5(8):e008378.

Bernhardt J, Speare S, Collier J, Churilov L, Thrift AG, Lindley R, et al. Reasons for non-recruitment to A Very Early Rehabilitation Trial (AVERT). International Journal of Stroke 2013;8 Suppl 1:41. [Ref 22301]

Bernhardt J, AVERT Trialists' Collaboration. A Very Early Rehabilitation Trial (AVERT): progress. International Journal of Stroke 2013;8 Suppl 1:41. [Ref 22300]

Bernhardt J, AVERT Trialists' Collaboration. A Very Early Rehabilitation Trial (AVERT): update. 22nd European Stroke Conference. 2013 May 28 - 31; London, UK. 2013. [Abst OAID109; Ref 20363]
Bernhardt J, AVERT Trialists' Collaboration. A very early stroke rehabilitation trial (AVERT): an ongoing phase III randomised controlled trial. Neurorehabilitation and Neural Repair 2012;26(6):804. [Abst 643; Ref 19206]

Bernhardt J, AVERT Trialists' Collaboration. AVERT: ongoing phase III, multicentre, international trial of a very early rehabilitation after stroke. 21st European Stroke Conference. 2012 May 22-25; Lisbon, Portugal. 2012. [Abst OAID 31; Ref 18874]

Bernhardt J, AVERT Trialists' Collaboration. AVERT: ongoing phase III, multicentre, international trial of very early rehabilitation after stroke. 8th World Stroke Congress. 2013 October 10-13; Brasilia, Brazil. 2013. [Abst 187; Ref 20500]

Bernhardt J, AVERT Trialists' Collaboration. Ongoing international trial of very early stroke rehabilitation (AVERT): progress. International Stroke Conference. 2014 February 12-14; San Diego, California. 2014. [Abst. CT P25; Ref 21738]

Chen R. Taking the ivory tower of academic research into the clinical world: clinicians' experiences of participating in an international rehabilitation trial (AVERT). Internal Medicine Journal 2008;38 Suppl 4:A75. [Ref 13650]

Collier J. Can more stroke patients be recruited into an ongoing trial of very early rehabilitation (AVERT)?. International Journal of Stroke 2010;5 Suppl 1:31. [Abst 0177; Ref 16659]

Collier J. The practical challenges of ensuring high quality data for a large multinational stroke rehabilitation trial (AVERT). International Journal of Stroke 2010;5 Suppl 1:4. [Abst 10; Ref 16637]

Collier J, Lindley R, Dewey H, Thrift A, Langhorne P, Donnan G, et al. Preliminary safety for stroke patients treated with alteplase in a very early rehabilitation trial (AVERT phase III). Cerebrovascular Diseases 2010;29 Suppl 2:201. [Abst 307; Ref 16331]

Collier J, Speare S, Churilov L, Bernhardt J. What stops patients being recruited to an early rehabilitation trial? Preliminary results from an ongoing phase III RCT (AVERT). Cerebrovascular Diseases 2010;29 Suppl 2:60. [Abst 3; Ref 16311]

Collier J, Thrift A, McQuinn A, Fu C, Grealy S, Bernhardt J. Implimentation of a randomized controlled trial of very early mobilization does not change standard stroke unit care. Physiotherapy 2007;93 Suppl 1:S128. [Abst 3280; Ref 16552]

Collier J, AVERT Trialists' Collaboration. International ongoing stroke trial of very early rehabilitation (AVERT). International Journal of Stroke 2013;8 Suppl 1:33. [Ref 22302]

Collier JM, Bernhardt J, Dewey H, Thrift A, Lindley R, Donnan G, et al. A Very Early Rehabilitation Trial (AVERT): ongoing phase III trial. Efficacy \& cost effectiveness study. 19th European Stroke Conference 2010. 2010 May 25-28; Barcelona, Spain. 2010. [Abst OAID52; Ref 16148]

Craig LE, Langhorne P, Wu O, Walters MR, Ritchie CK, Smith LN. AVERT Scotland: introducing a very early rehabilitation trial 
(AVERT) to Scotland. Cerebrovascular Diseases 2009;27 Suppl 6:241. [Abst 3; Ref 14779]

Cumming T, Linden T, Bernhardt J. There is no excuse for ignoring cognition: using the Montreal Cognitive Assessment is feasible in a large stroke trial. Stroke 2011;42(3):e56. [Abst 42; Ref 17606]

Cumming TB, Bernhardt J, Linden T. The Montreal Cognitive Assessment. Short cognitive evaluation in a large stroke trial. Stroke 2011;42:2642-4. [Ref 17738]

Dagonnier M, Muhl L, Kulin J, Churilov L, Dewey H, Linden T, et al. Early mobilization after thrombolysis (rt-PA) in acute stroke: are rt-PA treated patients enrolled in a trial of early mobilization (AVERT) different from those that are not?. Cerebrovascular Diseases 2013;35 Suppl 3:764. [Abst 792; Ref 21154]

Donnan G. Stroke rehabilitation: How early should it begin?. Stroke Rehab 2006. Evidence for Stroke Rehabilitation - Bridging into the Future. 2006 April 26-28; Göteborg, Sweden. 2006. [Ref 13349]

Ellery F, Borschmann K, Bernhardt J. Trials - the tribulations and the truths: recruiting hospitals to a very early rehabilitation trial (AVERT). International Journal of Stroke 2010;5 Suppl 1:20. [Abst O114; Ref 16647]

Ellery F, Borschmann K, Bernhardt J, Morrison R, Langhorne P. Trials, tribulations and the truth: recruiting hospitals to a very early rehabilitation trial (AVERT - international). Neurorehabilitation and Neural Repair 2012;26(6):742-3. [Abst 228; Ref 19202]

Ferris M. Collecting high quality data during the AVERT trial. International Journal of Stroke 2012;7 Suppl 1:60-1. [Ref 19993]

Langhorne P, Ashburn A, Rodgers $\mathrm{H}$, Wu O, Lennon S, Bernhardt J, et al. [A Very Early Rehabilitation Trial (AVERT) UK: update]. 8th UK Stroke Forum Conference. 2013 December 3-5; Harrogate, UK. 2013:65. [Abst OG20; Ref 21337]

Muhl L, Kulin J, Daggonier M, Churilov L, Dewey H, Bernhardt J, et al. Early mobilization after thrombolysis (rt-PA) in acute stroke: are rt-PA treated patients enrolled in a trial of early mobilization (AVERT) different from those who are not? International Journal of Stroke 2013;8 Suppl 1:19. [Ref 22297]

Muhl L, Kulin J, Dagonnier M, Churilov L, Dewey H, Linden T, et al. Early mobilization after thrombolysis (rt-PA) in acute stroke: are rt-PA treated patients enrolled in a trial of early mobilization (AVERT) different from those who are not?. Stroke 2014;45 Suppl 1:ATP104. [Abst ATP104; Ref 21898]

NCT01846247. A very early rehabilitation trial (AVERT). clinicaltrials.gov/ct2/show/NCT01846247 (first received 3 May 2013). [Ref 20367]

O'Neil N, Collier J, Quang L, Bernhardt J. Collaboration works! Linking stroke researchers and software engineering students to create AVERT online. Internal Medicine Journal 2006;36:A13. [Ref 10726]
Shannon M. Stroke research (AVERT) in an acute private Australian hospital: early experience. International Journal of Stroke 2013;8 Suppl 2:7. [Ref 22304]

Speare S, Collier J, Churilov L, Berhardt J, AVERT Trialist's Collaboration. What are the main reasons for exclusion from an early rehabilitation trial (AVERT)?. Neurorehabilitation and Neural Repair 2012;26(6):742. [Abst 224; Ref 19200]

Speare S, Collier J, Churilov L, Thrift A, Lindley R, Donnan G, et al. Exclusion from an early rehabilitation trial (AVERT): an exploratory analysis. International Journal of Stroke 2013;8 Suppl 2:11. [Ref 22303]

Tan D, Ahmad MT. A very early rehabilitation trial (AVERT) in Asia. International Journal of Stroke 2012;7 Suppl 1:61. [Ref 19995]

Zhao H, Collier JM, Quah DM, Purvis T, Bernhardt J. The Modified Rankin Scale as a measure of acute stroke disability. Internal Medicine Journal 2008;38 Suppl 4:A111. [Abst Poster 102; Ref 13639]

\section{Chippala 2015a \{published data only\}}

Chippala P, Sharma R. Effect of very early mobilisation on functional status in patients with acute stroke: a single-blind randomized controlled trial. Clinical Rehabilitation 2015;12:1-7.

\section{Chippala 2015b \{published data only\}}

Chippala P, Sharma R. Effect of very early mobilisation on disability and adverse events in the first 3 months post stroke: a single-blind, randomised controlled trial. International Journal of Health Sciences and Research 2015;5(10):166-74.

\section{Langhorne 2010 \{published and unpublished data}

Knight A, Langhorne P, Stott D, Bernhardt J, Barer D, Watkins C. Very early rehabilitation or intensive telemetry after stroke (VERITAS): a pilot randomised trial. 16th European Stroke Conference; 2007 May 29 - June 1; Glasgow, UK,. 2007.

Langhorne P, Knight A, Stott DJ, Bernhardt J, Barer D, Watkins C. Very early rehabilitation or intensive telemetry after stroke (VERITAS): pilot randomised trial. International Journal of Stroke 2008;3 Suppl 1:241. [Abst PO01-546; Ref 13927]

Langhorne P, Knight A, Stott DJ, Bernhardt J, Watkins CL, Barer D. Very early rehabilitation or intensive telemetry after stroke (VERITAS): a pilot randomised trial. Cerebrovascular Diseases 2008;25 Suppl 2:168. [Abst 10; Ref 13570]

Langhorne P, Stott D. Letter regarding article: Very Early Mobilization After Stroke Fast-Tracks Return to Walking: Further Results From the Phase II AVERT Randomized Controlled Trial. Stroke 2011;42:e376. [Ref 17585]

* Langhorne P, Stott D, Knight A, Bernhardt J, Barer D, Watkins $C$. Very early rehabilitation or intensive telemetry after stroke: a pilot randomised trial. Cerebrovascular Diseases 2010;29:352-60. [Ref 16171]

\section{Morreale 2016 \{published data only\}}

Morreale M, Marchione P, Pili A, Lauta A, Castiglia SF, Spallone A, et al. Early versus delayed rehabilitation treatment in 
hemiplegic patients with ischemic stroke: proprioceptive or cognitive approach?. European Journal of Physical and Rehabilitation Medicine 2016;52(1):81-9.

\section{Morreale 2016 CTE \{published data only\}}

Morreale M, Marchione P, Pili A, Lauta A, Castiglia SF, Spallone A, et al. Early versus delayed rehabilitation treatment in hemiplegic patients with ischemic stroke: proprioceptive or cognitive approach?. European Journal of Physical and Rehabilitation Medicine 2016;52(1):81-9.

\section{Morreale 2016 PNF \{published data only\}}

Morreale M, Marchione P, Pili A, Lauta A, Castiglia SF, Spallone A, et al. Early versus delayed rehabilitation treatment in hemiplegic patients with ischemic stroke: proprioceptive or cognitive approach?. European Journal of Physical and Rehabilitation Medicine 2016;52(1):81-9.

\section{Poletto 2015 \{published data only\}}

NCT01694992. Very Early Rehabilitation in acute Ischemic Stroke (VERIS-Brazil). clinicaltrials.gov/ct2/show/NCT01694992 (first received 27 September 2012).

* Poletto SR, Rebello LC, Valenca MJM, Rossato D, Almeida AG, Brondani R, et al. Early mobilization in ischemic stroke: a pilot randomized trial of safety and feasibility in a public hospital in Brazil. Cerebrovascular Diseases Extra 2015;5:31-40.

\section{SEVEL 2016 \{published and unpublished data\}}

* Herisson FE, Godard S, Volteau C, Le Blanc E, Guillon B, Gaudron M. Early sitting in ischemic stroke patients (SEVEL): a randomized controlled trial. PLOS ONE 2016;11(3):e0149466. [DOI: 10.1371/journal.pone.0149466]

NCT01573299. Ischemic stroke and early vertical positioning (SEVEL). clinicaltrials.gov/ct2/show/NCT01573299 (first received 9 April 2012).

\section{Sundseth 2012 \{published and unpublished data\}}

NCT00832351. AKershus Early Mobilisation In Stroke study (AKEMIS). clinicaltrials.gov/ct2/show/NCT00832351 (first received 30 January 2009). [Ref 15894]

Sundseth A, Thommessen B, Ronning M. Mobilisation within 24 hours of acute stroke. A randomised controlled trial, Akerhus mobilisation in stroke study (AKEMIS). Cerebrovascular Diseases 2012;33 Suppl 2:623-4. [Abst 599; Ref 19408]

Sundseth A, Thommessen B, Ronning OM. Early mobilisation after stroke. 17th European Stroke Conference. 2008 May 13-16; Nice, France. 2008. [Abst 76; Ref 13227]

* Sundseth A, Thommessen B, Ronning OM. Outcome after mobilization within 24 hours of acute stroke. Stroke 2012;43:2389-94. [Ref 19311]

\section{References to studies excluded from this review \\ AMOBES 2017 \{published data only\}}

Yelnik AP, Quintaine V, Andriantsifanetra C, Wannepain M, Reiner $\mathrm{P}$, Marnef H, AMOBES group. AMOBES (active mobility very early after stroke). A randomized controlled trial. Stroke 2017;48:400-5.

\section{Asberg 1989 \{published data only\}}

Asberg KH. Orthostatic tolerance training of stroke patients in general medical wards: an experimental study. Scandinavian Journal of Rehabilitation Medicine 1989;21:179-85.

Chu 2003 \{published data only\}

Chu P. Effect of early facilitation techniques on motor function of stroke patients. Chinese Journal of Clinical Rehabilitation 2003;4(15):1189.

\section{Di Lauro 2003 \{published data only\}}

Di Lauro A, Pellegrino L, Savastano G, Ferraro C, Fusco M, Balzarano $\mathrm{F}$, et al. A randomized trial on the efficacy of intensive rehabilitation in the acute phase of ischemic stroke. Journal of Neurology 2003;250:1206-8

Diserens 2010 \{published and unpublished data\}

Diserens K, Moreira T, Hirt L, Faouzi M, Grujic J, Bieler G, et al. Early mobilization out of bed after ischaemic stroke reduces severe complications but not cerebral blood flow: a randomized controlled pilot trial. Clinical Rehabilitation 2012;26(5):451-9. [[Ref 18934]]

* Diserens K, Moreira T, Hirt L, Grujic J, Bieler G, Vaudens P, et al. Early mobilisation out of bed after ischemic stroke reduces complications but not cerebral blood flow. Cerebrovascular Diseases 2010;29 Suppl 2:246. [Abst 632; Ref 16416]

Duan 2006 \{published data only\}

Duan G. Early rehabilitation nursing in patients with stroke. Medicine World 2006;6:139-41.

Fang 2001a \{published data only\}

Fang DH, Wang MB, Hu DM, Liu XQ. A study of early rehabilitation of stroke. Chinese Journal of Rehabilitation Medicine 2001;16(5):266-72. [[Ref 7422]]

Fang 2001b \{published data only\}

Fang Y, Chen X, Li H, Lin J, Huang J, Zeng J, et al. A study on early onset of rehabilitation after stroke and factors affecting functional outcome. Internal Medicine Journal 2003;33(7):A53. [Ref 9827]

Fang Y, Chen X, Li H, Lin J, Huang R, Zeng J. A study on additional early physiotherapy after stroke and factors affecting functional recovery. Clinical Rehabilitation 2003;17:608-17. [Ref 7794]

Fang Y-N, Li H, Zeng J-S, Chen X-H, Ma M-M, Lin J-W, et al. Analysis of the factors affecting the functional rehabilitation of senile stroke patients. Chinese Journal of Clinical Rehabilitation 2004;8(10):1818-20. [Ref 9388]

* Fang YN, Huang RX, Li H, Lin JW, Zeng JS. A study on early onset of rehabilitation interventions after stroke and factors affecting functional outcome. Hong Kong Medical Journal 2001;7(4 Suppl 1):25. [Ref 7803] 
Gong 2003 \{published data only\}

Gong S, Zhang J, Yu K. Effect of early rehabilitation training on daily life activity of patients with hemiplegia after stroke. Chinese Journal of Clinical Rehabilitation 2003;7(5):848.

\section{Gorbunov 2003 \{published data only\}}

Gorbunov FE, Kochetkov AV. Sanatorium stage of early rehabilitation of patients with prior acute ischaemic attack [Sanatornyi etap rannei reabilitasii bol'nykh, perenesshikh ostrye narusheniia mozgovogo krovoobrashcheniia]. Voprosy Kurortologii, Fizioterapii i Lechebnoi Fizicheskoi Kultury 2003;1:25-30.

\section{Gu 2006 \{published data only\}}

Gu H-Y, Li K. The effect of early rehabilitation therapy on the movement function of limbs in patients with cerebral stroke. China Tropical Medicine 2006;6(12):2213-4.

\section{Guan 2001 \{published data only\}}

Guan J, Guo K, Zhu Y. Investigates the effects of early rehabilitation and nursing management in the patients suffering stroke. Modern Rehabilitation 2001;5(6):50-1.

\section{Hamrin 1982 \{published data only\}}

* Hamrin E. Il. Early activation in stroke: does it make a difference?. Scandinavian Journal of Rehabilitation Medicine 1982;14:101-9.

Hamrin E. III. One year after stroke: a follow-up of an experimental study. Scandinavian Journal of Rehabilitation Medicine 1982;14:111-6.

\section{Hara 2001 \{published data only\}}

Hara H. Effective execution of early stroke rehabilitation in the acute hospital. Japanese Journal of Rehabilitation Medicine 2001;38(7):532.

\section{Huang 2001 \{published data only\}}

Huang D, Mao Y, Xu G. Value of early rehabilitation on severe stroke patients in intensive care unit (ICU). Chinese Journal of Physical Medicine and Rehabilitation 2001;23(6):328-30.

\section{Huang 2003 \{published data only\}}

Huang X, Mao L, Sun Y. A clinical study of early rehabilitation of hemiplegics after stroke. Medical Journal of Chinese People Health 2003;15:460-1.

\section{Ishida 2001 \{published data only\}}

Ishida A, Tanaka H, Toyokura M, Izumi S. Early rehabilitative intervention for stroke - prospective study. 1st World Congress of the International Society of Physical Rehabilitation Medicine (ISPRM I). 2001 July 7-13; Amsterdam, The Netherlands. 2001:500-4

\section{Kreisel 2005 \{published data only\}}

Kreisel SH, Bazner H, Hennerici MG. Intensive rehabilitation in the acute phase of stroke: positive or negative effects on outcome?. Cerebrovascular Diseases 2005;19 Suppl 2:92.

\section{Li 1999 \{published data only\}}

Li M, Chen Y, Jiang J. Early rehabilitation of acute hemiplegic stroke: experience of 30 cases. Chinese Journal of Practical Internal Medicine 1999;19(6):352-3.

\section{Li 2003 \{published data only\}}

Li F. Impact of early rehabilitation nursing on paralyzed limbs in patients with cerebral haemorrhage. Chinese Journal of Clinical Rehabilitation 2003;7:841.

Li 2004 \{published data only\}

Li W-D, Huang B-B. Effects of the treatment for post-stroke depression on the recovery of motor function and ability of daily living. Chinese Journal of Clinical Rehabilitation 2004;8(13):2410-1.

Lin 2005 \{published data only\}

Lin C, Mo W. Effect of early rehabilitation nursing on quality of life patients with stroke. Modern Nursing 2005;11(12):968-9.

\section{Liu 2001b \{published data only\}}

Liu X. Effect on use of systematic recovery nursing care in earlier stage of acute cerebral infarction patients accompanied with hemiparalysis. Chinese Nursing Research 2001;15(4):210-1. [Ref 13136]

\section{Liu 2003b \{published data only\}}

Liu G, Zhang H. Early rehabilitation and prognosis of post-stroke hemiplegic patients. Chinese Journal of Clinical Rehabilitation 2003;7(3):506. [Ref 9717]

\section{Liu 2004 \{published data only\}}

Liu Z, Guan S, Song L, Zheng W. Effects of functional electrical stimulation on the integral of motor function of lower limbs in patients with stroke hemiplegia. Chinese Journal of Clinical Rehabilitation 2004;8(31):6824-5.

\section{Marshall 2011 \{published data only\}}

Marshall RS, Cheung YC, Bassile CC, Evensen LA, Chen R, Perez V, et al. Acute stroke combined with early neurorehabilitation treatment (ASCENT): a pilot safety-feasibility study. Cerebrovascular Diseases 2010;31:191.

\section{Miskovic 2004 \{published data only\}}

Miskovic M, Okiljevic D, Perisic O, Durovic AA, Markovic L, Raicevic R. The significance of early physical therapy in prevention of complications in patients with stroke. Stroke 2004;36(6):e310.

\section{Pan 2004 \{published data only\}}

Pan C-H, He J-Q, Pu S-X, Wang X-L, Gao C. Effects of early rehabilitation therapy on the motor function of limbs and ability of daily living in patients with hemiplegia after stroke. Chinese Journal of Clinical Rehabilitation 2004;8(13):2404-5.

\section{Qian 2003 \{published data only\}}

Qian H, Huang Y. Analysis of related factor with early rehabilitation of hemiplegic extremity in aged patients with stroke. Journal of Clinical Healthcare 2003;6(2):96-8. 
Qian 2004 \{published data only\}

Qian K-L, Wang T. Effect of early rehabilitation therapy on short and long term functional assessment in hemiplegic patients after stroke. Chinese Journal of Clinical Rehabilitation 2004;8(25):520-1.

\section{Raicevic 2000 \{published data only\}}

Miskovic M, Okiljevic D, Perisic O, Durovic A, Markovic L, Raicevic $R$. The significance of early physical therapy in prevention of complications in patients with stroke. Stroke 2004;35(6):e310.

* Raicevic R, Jovicic A, Marenovic T, Jevdjic J, Surbatovic M, Markovic $L$, et al. The early physical therapy in patients with ischemic brain disease in prevention of bacterial complications. European Journal of Neurology 2000;7 Suppl 3:98.

\section{Richards 1993 \{published data only\}}

Richards CL, Malouin F, Wood-Dauphinee S, Williams JI, Bouchard JP, Brunet D. Task-specific physical therapy for optimization of gait recovery in acute stroke patients. Archives of Physical Medicine and Rehabilitation 1993;74(6):612-20.

\section{Sankara Kumaran 2013 \{published and unpublished data\}} Kumaran PS, Tamil Vanan M. Effect of early mobilisation training on gross motor function and functional outcome in hemiparetic stroke patients. International Journal of Pharmacy and Technology 2013;5(3):5637-50.

\section{Song 2005 \{published data only\}}

Song Y. A study on effect of early rehabilitation nursing on patients with hemiplegia caused by acute cerebral stroke. Journal of Qilu Nursing 2006;11(9A):1189-90.

\section{Sun 2002 \{published data only\}}

Sun RH, Dong LQ, Li K, Hu YR, Duan SR, Wang DS. Early rehabilitation therapy for acute stroke. Chinese Journal of Geriatric Cardiovascular and Cerebrovascular Diseases 2002;4(4):230.

\section{Toyota 2001 \{published data only\}}

Toyota A, Shima T, Nishida M, Yamane K, Hatayama T, Yamanaka C. Early rehabilitation for stroke patients. Journal of Stroke and Cerebrovascular Diseases 2000;9:109-10.

\section{Truscott 1974 \{published data only\}}

Truscott BL, Kretschmann CM, Toole JF, Pajak T. Early rehabilitative care in community hospitals: effect on quality of survivorship following a stroke. Stroke 1974;5:623-9.

\section{Wang 2004 \{published data only\}}

Wang Q-R, Gan Z-R, Lu H, Hu X, Liu H-L, Cai C-Q, et al. Effect of early exercise therapy on the recovery of motor function in patients with cerebral infarction and the changes of somatosensory evoked potentials. Zhongguo Linchuang Kangfu 2004;8(28):6023-5.

\section{Wang 2005 \{published data only\}}

Wang W, Xue Y, Ren J, Wei J. Effect of early rehabilitation on motor and cognitive function of hemiplegia after stroke. Chinese Journal of Rehabilitation Theory and Practice 2006;12:413-4.
Wang 2006 \{published data only\}

Wang B, Liu W, Wu J. The effect of early rehabilitation in stroke unit on recovery of limbs ability of patients. Journal of Qilu Nursing 2006;12(8):1411-2.

\section{Wu 2012 \{published data only\}}

Wu D-Y, Guo M, Gao Y-S, Kang Y-H, Guo J-C, Jiang X-L, et al. Clinical effects of comprehensive therapy of early psychological intervention and rehabilitation training on neurological rehabilitation of patients with acute stroke. Asian Pacific Journal of Tropical Medicine 2012;12:914-6. [Ref 20798]

\section{Xi 2003 \{published data only\}}

Xi M, Zhang Y, Xu Z, Qin Y. Early rehabilitation nursing of hemiplegia patients with acute stroke. Nursing Journal of the Chinese People's Liberation Army 2003;20:11-3.

\section{Xiao 2000 \{published data only\}}

Xiao Y. Effect of early stage rehabilitation nursing on ability of daily life in patients with cerebral apoplexy. Shanxi Nursing Journal 2000;14(3):122-3. [Ref 13135]

Xiao 2004 \{published data only\}

Xiao W-Z, Fan D-S, Fu G-M, Li J, Zhang X-Y, Sui W. The clinical economic evaluation of the early rehabilitation treatment for the stroke patients. Chinese Journal of Clinical Rehabilitation 2004;8(10):1811-3.

Xie 2003a \{published data only\}

Xie S, Zhu M, Zhang X. Effect of early rehabilitation nursing on ability of daily living in patients with stroke. Chinese Journal of Clinical Rehabilitation 2003;7(1):143. [Ref 9725]

\section{Xue 2004 \{published data only\}}

Xue W, Zhang S-Q, Xiang L. Evaluation of curative effect of early rehabilitation treatment in patients with cerebral hemorrhage by using National Institute of Health Stroke Scale and Mini Mental State Examination. Chinese Journal of Clinical Rehabilitation 2004;8(25):5222-3.

\section{Xue 2006 \{published data only\}}

Xue J, Bai L, Guo QR, Yang CR, Lu J. Efficacy of early intervention of motor relearning program on post-stroke hemiplegia: a randomized controlled observation. Neural Regeneration Research 2006;1(3):277-9.

\section{Xue 2008 \{published data only\}}

Xu Y. The effect of early intensive exercise on patients with hemiplegia. Journal of Rehabilitation Medicine 2008;Suppl 46:75. [Abst PP001-065; Ref 15479]

Zeng 2004 \{published data only\}

Zeng F-J, Chen Z-H, Jiang Q-H, Yang X-Z, Chen Y, Mu Z-W, et al. Effects of notoginseng extract and early rehabilitation on the microcirculation and hemorheology in patients with cerebral infarction. Chinese Journal of Clinical Rehabilitation 2004;8(31):7078-80.

\section{Zhang 1998 \{published data only\}}

Zhang XY. The effects of early rehabilitation on hemiplegic stroke patients. Heilongjiang Nursing Journal 1998;4(11):1-2. 
Zhang 2001 \{published data only\}

Zhang W, Li Y. Observation for therapeutic effects for early rehabilitation of acute hemiplegic stroke. Journal of Henan Medical College for Staff and Workers 2001;13:267-8.

\section{Zhao 2003 \{published data only\}}

Zhao F, Wang L, Tian G, Zhou J, Han J. Early rehabilitation intervention promoting ability of daily living in acute stroke patients. Chinese Journal of Clinical Rehabilitation 2003;7(5):851.

\section{Zheng 2004a \{published data only\}}

Zheng L, Mei Y, Xing $\mathrm{H}$, Chen Y. Effect of three-stage rehabilitation scheme on the quality of life in stroke patients. Chinese Journal of Clinical Rehabilitation 2004;8(28):6020-2. [Ref 13184]

\section{References to studies awaiting assessment}

Izumi 2001 \{published data only\}

Izumi SI. Stroke rehabilitation at university hospitals (1) Early rehabilitative intervention for stroke, a randomized control study. Japanese Journal of Rehabilitation Medicine 2001;38(7):535.

Liu 2010e \{published data only (unpublished sought but not used)\} ChiCTR-TRC-13004039. Randomized controlled trial of very early rehabilitation after intracerebral hemorrhage stroke: two years follow-up of stroke survivors outcomes. www.chictr.org.cn/showprojen.aspx?proj=5528 (first received 20 November 2013). [Ref 22755]

* Liu N, Cadilhac DA, Andrew NE, Zeng L, Zongfang L, Li J, et al. Randomized controlled trial of early rehabilitation after intracerebral hemorrhage stroke: difference in outcomes within 6 months of stroke. Stroke 2014;45:3502-07.

\section{Nilsson 2003 \{published data only\}}

Nilsson L, Carlsson J, Danielsson A, Fugl Meyer A, Hellstrom K, Kristensen $L$, et al. Walking training of patients with hemiparesis at an early stage after stroke. 14th International Congress of The World Confederation for Physical Therapy. 2003.

Skevin 2009 \{published data only\}

Skevin AJ, Jevtic M, Veljkovic M, Markovic VG. Importance of an early rehabilitation program for hemiplegics after cerebrovascular insult. Medicus 2007;8(3):102-8.

\section{Song 2010 \{published data only\}}

Song YX. Clinical observation of time window of rehabilitation of cerebral infarction. Journal of Dalian Medical University 2010;32(4):455-7.

\section{Xu 2001 \{published data only\}}

Xu J, Geng Q. The importance of early rehabilitation on ability of daily life in patients with cerebral apoplexy. 1st International Congress of International Society of Physical and Rehabilitation Medicine (ISPRM I). 2001 July 7-13; Amsterdam, The Netherlands. 2001.

\section{Zheng 2004 \{published data only\}}

Zheng $\mathrm{Q}$. An investigation on early stage rehabilitation exercise to promote motor function recovery in patients with cerebral apoplexy. Chinese Nursing Research 2004;18(12B):2179-81.

Zielke 2003 \{published data only\}

Zielke DR. The effect of partial body weight supported treadmill training on gait rehabilitation in early acute stroke patients: preliminary data. Journal of Neurologic Physical Therapy 2003;27:177.

\section{References to ongoing studies \\ AVERT-DOSE 2017 \{unpublished data only\}}

Bernhardt J, et al. AVERT-DOSE (Determining Optimal early rehabilitation after StrokE): a multi-arm covariateadjusted, response-adaptive randomised controlled trial. www.nhmrc.gov.au/grants-funding/outcomes-funding-rounds (first received 6 December 2017).

\section{ChiCTR-ICR-15005992 \{published data only\}}

ChiCTR-ICR-15005992. Effect of early and intensive rehabilitation on functional recovery after stroke. www.chictr.org.cn/showproj.aspx?proj=10455 (first received 11 February 2015).

\section{ChicTR-IPR-16008652 \{published data only\}}

ChiCTR-IPR-16008652. Efficacy and safety of very early rehabilitation within $24 \mathrm{~h}$ of ischemic stroke with small-artery occlusion. www.chictr.org.cn/showprojen.aspx?proj=14502 (first received 15 June 2016).

\section{ChiCTR-TRC-08000201 \{published data only\}}

ChiCTR-TRC-08000201. The effect of early mobilization for stroke patients. www.chictr.org.cn/showprojen.aspx?proj=9324 (first received 29 November 2008). [Ref 15906]

\section{Additional references}

\section{Adams 2003}

Adams HJ, Adams R, Brott T, del Zoppo G, Furlan A, Goldstein L, et al. Guidelines for the early management of patients with ischemic stroke: a scientific statement from the Stroke Council of the American Stroke Association. Stroke 2003;34:1056-83.

\section{Allen 1999}

Allen C, Glasziou P, del Mar C. Bed rest: a potentially harmful treatment needing more careful evaluation. Lancet 1999;354:1229-33.

\section{Anderson 2017}

Anderson CS, Arima H, Lavados P, Billot L, Hackett ML, Olavarría VV, HeadPoST Investigators and Coordinators. Clusterrandomized, crossover trial of head positioning in acute stroke. New England Journal of Medicine 2017;376:2437-47. [for protocol; see Muñoz-Venturelli 2015] 


\section{Barber 2004}

Barber M, Stott D, Lanhorne P. An internationally agreed definition of progressing stroke. Cerebrovascular Diseases 2004;18:255-6.

\section{Bernhardt 2004}

Bernhardt J, Dewey HM, Thrift AG, Donnan GA. Inactive and alone: physical activity within the first 14 days of acute stroke unit care. Stroke 2004;35:1005-9.

\section{Bernhardt 2007}

Bernhardt J, Indredavik B, Dewey H, Langhorne P, Lindley R, Donnan $\mathrm{G}$, et al. Mobilisation 'in bed' is not mobilisation. Cerebrovascular Diseases 2007;24:157-8.

\section{Bernhardt 2015}

Bernhardt J, English C, Johnson L, Cumming TB. Early mobilization after stroke: early adoption but limited evidence. Stroke 2015;46:1141-6.

\section{Bernhardt 2017}

Bernhardt J. Editorial. International Journal of Stroke 2017;12(5):443.

\section{Brott 1989}

Brott T, Adams H, Olinger C, Marler J, Barsan W, Biller J, et al. Measurements of acute cerebral infarction: a clinical examination scale. Stroke 1989;20:864-70.

\section{Diserens 2006}

Diserens K, Michel P, Bogousslavsky J. Early mobilization after stroke: review of the literature. Cerebrovascular Diseases 2006;22:183-90.

\section{Gao 2001}

Gao C, Pu S, Zhu D. Effects of early rehabilitation on motor function of upper and lower extremities and activities of daily living in patients with hemiplegia after stroke. Chinese Journal of Rehabilitation Medicine 2001;16:27-9.

\section{Higgins 2011}

Higgins JPT, Green S, editor(s). Cochrane Handbook for Systematic Reviews of Interventions Version 5.1.0 (updated March 2011). The Cochrane Collaboration, 2011. Available from handbook.cochrane.org.

\section{Hoffman 2014}

Hoffmann TC, Glasziou PP, Boutron I, Milne R, Perera R, Moher D, et al. Better reporting of interventions: template for intervention description and replication (TIDieR) checklist and guide. BMJ 2014; Vol. 348:g1687. [DOI: 10.1136/bmj.g1687]

\section{Indredavik 1999}

Indredavik B, Bakke PRT, Slordahl SA, Rokseth R, Haheim LL. Treatment in a combined acute and rehabilitation stroke unit: which aspects are most important?. Stroke 1999;30:917-23.

\section{Johansson 2000}

Johansson BB. Brain plasticity and stroke rehabilitation: the Willis lecture. Stroke 2000;31:223-30.

\section{Krakauer 2012}

Krakauer JW, Carmichael ST, Corbett D, Wittenberg GF. Getting neurorehabilitation right: what can be learned from animal models?. Neurorehabilitation and Neural Repair 2012;26:923-31.

\section{Langhorne 2000}

Langhorne P, Stott DJ, Robertson L, MacDonald J, Jones L, McAlpine C, et al. Medical complications after stroke: a multicenter study. Stroke 2000;31:1223-9.

\section{Langhorne 2012}

Langhorne P, de Villiers L, Pandian JD. Applicability of strokeunit care to low-income and middle-income countries. Lancet Neurology 2012;11:341-8.

\section{Li 2002}

Li J, Zhang H, Mi S. Effects of early rehabilitation training on motor function of upper and lower extremities and activities of daily living in patients with hemiplegia after stroke. Journal of Navy Medicine 2002;23:35-7.

\section{Lu 2006}

Lu G, Ades A. Assessing evidence inconsistency in mixed treatment comparisons. Journal of the American Statistical Association 2006;101(474):447-59.

\section{Lynch 2014}

Lynch E, Hillier S, Cadilhac D. When should physical rehabilitation commence after stroke: a systematic review. International Journal of Stroke 2014;9:468-78.

\section{Moher 2001}

Moher D, Schulz KF, Altman DG, CONSORT Group. The CONSORT statement: revised recommendations for improving the quality of reports of parallel-group randomised trials. Lancet 2001;357:1191-4.

\section{Murphy 2009}

Murphy $\mathrm{TH}$, Corbett D. Plasticity during stroke recovery: from synapse to behaviour. Nature Reviews Neuroscience 2009;10:861-72.

\section{Murray 2012}

Murray CJL, Vos T, Lozano R, Naghavi M, Flaxman AD, Michaud C, et al. Disability-adjusted life years (DALYs) for 291 diseases and injuries in 21 regions, 1990-2010: a systematic analysis for the Global Burden of Disease Study 2010. Lancet 2012;380:2197-223.

\section{Mutin-Carino 2014}

Mutin-Carnino M, Carnino A, Roffino S, Chopard A. Effect of muscle unloading, reloading and exercise on inflammation during a head-down bed rest. International Journal of Sports Medicine 2014;35:28-34.

\section{Muñoz-Venturelli 2015}

Muñoz-Venturelli P, Arima H, Lavados P, Brunser A, Peng B, Cui L, et al. Head Position in Stroke Trial (HeadPoST) - sittingup vs lying-flat positioning of patients with acute stroke: study protocol for a cluster randomised controlled trial. Trials 2015;16:256-67. [protocol for Anderson 2017] 


\section{NSF 2007}

Working Group of the National Stroke Foundation. Clinical guidelines for acute stroke management. www.nhmrc.gov.au/ publications 2007.

\section{Olavarria 2014}

Olavarría VV, Arima H, Anderson CS, Brunser AM, MuñozVenturelli P, Heritier S, et al. Head position and cerebral blood flow velocity in acute ischemic stroke: a systematic review and meta-analysis. Cerebrovascular Diseases 2014;37:401-8.

\section{Pekna 2012}

Pekna M, Pekny M, Nilsson M. Modulation of neural plasticity as a basis for stroke rehabilitation. Stroke 2012;4:2819-28.

\section{Pollock 2014a}

Pollock A, Baer G, Campbell P, Choo PL, Forster A, Morris J, Pomeroy VM, Langhorne P. Physical rehabilitation approaches for the recovery of function and mobility following stroke. Cochrane Database of Systematic Reviews 2014, Issue 4. [DOI: 10.1002/14651858.CD001920.pub3]

\section{Pollock 2014b}

Pollock A, Farmer SE, Brady MC, Langhorne P, Mead GE, Mehrholz J, van Wijck F. Interventions for improving upper limb function after stroke. Cochrane Database of Systematic Reviews 2014, Issue 11. [DOI: 10.1002/14651858.CD010820.pub2]

\section{Puhan 2014}

Puhan MA, Schünemann HJ, Murad MH, Li T, BrignardelloPetersen R, Singh JA, GRADE Working Group. A GRADE Working Group approach for rating the quality of treatment effect estimates from network meta-analysis. BMJ 2014;349:g5630. [DOI: 10.1136/bmj.g5630]

\section{RevMan 2014 [Computer program]}

Nordic Cochrane Centre, The Cochrane Collaboration. Review Manager 5 (RevMan 5). Version Version 5.3. Copenhagen: Nordic Cochrane Centre, The Cochrane Collaboration, 2014.

\section{Skarin 2011}

Skarin M, Bernhardt J, Sjoholm A, Nilsson M, Linden T. 'Better wear out sheets than shoes': a survey of stroke professionals' early mobilisation practices and concerns. International Journal of Stroke 2011;6:10-5.

\section{Song 2006}

Song F, Jerosch-Herold C, Holland R, de Lourdes Drachler M, Mares K, Harvey I. Statistical methods for analysing Barthel scores in trials of poststroke interventions: a review and computer simulations. Clinical Rehabilitation 2006;20:347-56.

\section{SUTC 2013}

Stroke Unit Trialists' Collaboration. Organised inpatient (stroke unit) care for stroke. Cochrane Database of Systematic Reviews 2013, Issue 9. [DOI: 10.1002/14651858.CD000197.pub3]

\section{Tonin 2017}

Tonin FS, Rotta I, Mendes AM, Pontarolo R. Network metaanalysis: a technique to gather evidence from direct and indirect comparisons. Pharmacy Practice 2017;15(1):943.

\section{Wan 2014}

Wan X, Wang W, Liu J, Tong T. Estimating the sample mean and standard deviation from the sample size, median, range and/or interquartile range. BMC Medical Research Methodology 2014;14:135.

\section{Whaley 2016}

Whaley M, Dusenbury W, Alexandrov AV, Tsivgoulis G, Alexandrov AW. To rest or mobilize ... when to start early mobilization in acute stroke: a systematic review. Proceedings of the International Stroke Congress. State-of-the-Science Stroke Nursing Symposium Oral Abstracts (ISC 2016). 2016.

\section{Xu 2017}

Xu T, Yu Y, Ou S, Liu X, Yuan J, Chen Y. Efficacy and safety of very early mobilization in patients with acute stroke: a systematic review and meta-analysis. Scientific Reports Scientific Reports 26 July 2017;7:Article number: 6550. [DOI: 10.1038/s41598-017-06871-z]

\section{Zhang 2014}

Zhang WW, Speare S, Churilov L, Thuy M, Donnan G, Bernhardt J. Stroke rehabilitation in China: a systematic review and meta-analysis. International Journal of Stroke 2014;9:494-502.

\section{References to other published versions of this review Bernhardt 2009}

Bernhardt J, Thuy MNT, Collier JM, Legg LA. Very early versus delayed mobilisation after stroke. Cochrane Database of Systematic Reviews 2009, Issue 1. [DOI: 10.1002/14651858.CD006187.pub2]

* Indicates the major publication for the study

\section{CHARACTERISTICS OF STUDIES}

Characteristics of included studies [ordered by study ID]

\section{AVERT || 2008}

Methods

RCT of very early and intensive mobilisation versus standard care

Computer-generated blocked randomization, stratified by stroke severity and clinical site, opaque envelopes

Blinded outcome assessment, participants blind to group 
AVERT II 2008 (Continued)

Participants
Stroke units in 2 large teaching hospitals in Melbourne, Australia

71 participants: 38 intervention, 33 control

Mean age: 74.7 years

$53.5 \%$ male

9 (13\%) haemorrhagic stroke

Mean NIHSS 10

$30(42 \%)$ had mild stroke (NIHSS 1 to 7 )

24 (33\%) moderate stroke (NIHSS 8 to 16$)$

$17(24 \%)$ severe stroke $($ NIHSS > 16)

Inclusion criteria: acute stroke patients admitted within 24 hours of symptom onset to a stroke unit, able to react to verbal commands, systolic BP 120 to $220 \mathrm{mmHg}$, oxygen saturation > 92\% (with or without supplementation), heart rate 40 to 100 , temperature $<38.5 \mathrm{C}$

Exclusion criteria: premorbid $\mathrm{mRS}>2$, deterioration within first hour of admission to stroke unit, direct admission to intensive care, concurrent progressive neurological disorder, acute coronary syndrome, severe heart failure, lower limb fracture, palliative care

\section{What}

Procedures: VEM plus standard care versus standard care alone. The VEM group commenced mobilisation (upright and out of bed at least twice a day) as soon as practical, aiming to have first mobilisation within 24 hours of stroke onset

\section{Who provided}

Mobilisation was delivered by a nurse-physiotherapist team

\section{How}

Mobilisation was delivered face-to-face by a nurse-physiotherapist team

\section{Where}

In the stroke unit

\section{When and how much}

VEM began within 24 hours and continued daily for 14 days post stroke, or until discharge

\section{Tailoring}

Participants were monitored during mobilisation within the first 3 days. Mobilisation could be halted in the event of significant physiological changes

\section{Modifications}

The intervention protocol was not changed during the trial. The type and amount of mobilisation was the same for infarct and haemorrhage

\section{How well}

Planned: time-to-first mobilisation and number of minutes of mobilisation (engaged in upright activities) were recorded

Actual: the median time-to-first mobilisation after symptom onset was 18.1 hours (IQR 12.8 to 21.5 ) in the VEM group and 30.8 hours (IQR 23.0 to 39.9 ) in the standard care group $(P<0.001)$. The total number of minutes (median, IQR) of mobilisation achieved in the VEM group was double that of standard care (VEM 167, 62 to 305 , standard care 69,31 to $115 ; \mathrm{P}=0.003$ )

Outcomes Mortality


AVERT II 2008 (Continued)

'Severe' adverse events

'Non-severe' adverse events

Deterioration in the first 7 days

Perceived exertion (Borg scale)

Total dose of mobilisation

Time from stroke onset to first mobilisation

mRS (disability or dependency)

Contamination (increase in mobility in a random sample of standard care participants)

Irritability, Depression, and Anxiety (IDA) scale

Assessment of Quality of Life scale

Notes

Trial ran from 2004 to 2006

Follow-up period: primary outcome 3 months, final follow-up 12 months

\section{Risk of bias}

\begin{tabular}{lll}
\hline Bias & Authors' judgement & Support for judgement \\
\hline $\begin{array}{l}\text { Random sequence genera- } \\
\text { tion (selection bias) }\end{array}$ & Low risk & $\begin{array}{l}\text { Quote: "We used computer-generated, blocked randomization procedures } \\
\text { with stratification by stroke severity and clinical site" }\end{array}$ \\
\hline $\begin{array}{l}\text { Allocation concealment } \\
\text { (selection bias) }\end{array}$ & Low risk & Quote: "Opaque envelopes concealed the group allocation" \\
\hline $\begin{array}{l}\text { Blinding of participants } \\
\text { and personnel (perfor- } \\
\text { mance bias) }\end{array}$ & Low risk & $\begin{array}{l}\text { Quote: "Patients were advised that they would be randomized to } 1 \text { of } 2 \text { styles } \\
\text { of rehabilitation, A or B. Trial therapists and nursing staff could not be blinded } \\
\text { to intervention group. To limit knowledge of VEM, interventions were conduct- } \\
\text { ed by dedicated trial staff out of sight of ward staff or behind closed curtains } \\
\text { wherever possible" }\end{array}$
\end{tabular}

\begin{tabular}{|c|c|c|}
\hline $\begin{array}{l}\text { Blinding of outcome as- } \\
\text { sessment (detection bias) } \\
\text { All outcomes }\end{array}$ & Low risk & $\begin{array}{l}\text { Quote: "All outcomes were assessed by a blinded assessor located off-site". } \\
\text { To assess whether protocols to blind the assessor to group were effective, } \\
\text { the blinded assessor was asked to select (forced choice) to which group they } \\
\text { thought the participant had been allocated. This procedure was introduced } \\
\text { in the third month of the study. A correct guess was made in } 34 / 56 \text { cases. This } \\
\text { was no better than chance }(P=0.25)\end{array}$ \\
\hline
\end{tabular}

Incomplete outcome data Low risk Only 2 participants $(2 / 71)$ lost to follow-up

(attrition bias)

All outcomes

Selective reporting (re- Low risk Primary outcome largely complete
porting bias)

porting bias)

\section{AVERT III 2015}

Methods AVERT III was a prospective, parallel group, assessor-blind, randomized, multi-centre, international clinical trial. Participants were randomized in a ratio of 1:1 to 2 groups: 1) very early and frequent mobilisation out of bed (VEM); and 2) usual care (UC)

Participants International, multicentre trial carried out in 56 stroke units in Australia, New Zealand, Singapore, Malaysia, and the UK (England, Scotland, Northern Ireland, and Wales) and recruiting acute stroke patients admitted to hospital within 24 hours of symptom onset

2104 participants recruited: 1054 intervention, 1050 control 
AVERT III 2015 (Continued)

Mean age: 72.5 years

$818 / 2104$ (39\%) men

258/2104 (12\%) haemorrhagic

Median NIHSS 7

$1170(55 \%)$ had mild stroke (NIHSS 1 to 7 )

$642(31 \%)$ moderate stroke (NIHSS 8 to 16$)$

$291(14 \%)$ severe stroke (NIHSS >16)

Thrombolysis treatment in $24 \%$

Inclusion criteria:

- first or recurrent, ischaemic or haemorrhagic stroke (but not transient ischaemic attack);

- recruited within 24 hours of onset of stroke symptoms;

- aged 18 years or more;

- admitted to a stroke care unit

- must at least react to verbal commands

Exclusion criteria:

- pre-stroke disability (mRS score of 3 to 5);

- deterioration in condition in the first hour of admission resulting in admission to ICU;

- decision for palliative treatment (e.g. those with devastating stroke) or immediate surgery or concurrent diagnosis of rapidly deteriorating comorbidity;

- unstable other medical condition that is judged by the investigator to impose a hazard to the patient by involvement in the trial;

- lower limb fracture at the time of stroke preventing the implementation of the mobilisation protocol;

- concurrent recruitment to another intervention trial;

- physiological instability (systolic blood pressure less than 110, or greater than $220 \mathrm{mmHg}$, oxygen saturation $<92 \%$ with oxygen supplementation, resting heart rate $<40$ or $>110$ beats per minute, temperature $>38.5^{\circ} \mathrm{C}$ )

Treatment with rtPA was not an exclusion criteria if the attending physician permitted mobilisation to commence within 24 hours of stroke onset

Materials: participant could receive usual care alone (UC) or very early and frequent mobilisation (VEM) in addition to usual care. The intervention followed a protocol (AVERT Intervention Protocol version 3, dated 25 April 2008) detailing a strict 'first time out of bed protocol'. The intervention protocol was only distributed to trial intervention staff and ethics committees in order to help maintain blinding and protect against treatment contamination in the trial. The intervention was task specific with a focus on recovery of standing and walking. The protocol allowed 4 levels of activity ranging from 4 transitions/day in severe patients (baseline NIHSS > 16) to 16 transitions/day in mild patients (baseline NIHSS 0 to 7)

Procedures: VEM participants commenced out-of-bed activity within 24 hours of stroke onset, to continue out of bed activity at a frequency and intensity guided by a detailed intervention protocol (available from the investigators)

\section{Who provided}

VEM was provided by physiotherapy and nursing staff trained in study procedures

\section{How}

VEM was provided face-to-face by physiotherapy and nursing staff

\section{Where}




\section{When and how much}

VEM began within 24 hours and lasted for 14 days or until the participant was discharged from stroke unit care. The VEM intervention included 3 key elements: 1 ) begin within 24 hours of stroke onset; 2) focus on sitting, standing, and walking (i.e. out-of-bed) activity; and 3) result in at least 3 out-of-bed sessions in additional to usual care. Participants assigned to VEM were assisted to continue out-of-bed activity at a dose guided by a detailed intervention protocol (see above)

\section{Tailoring}

The intervention followed a protocol that outlined the safety ranges for blood pressure, heart rate, oxygen saturation and temperature prior to first mobilisation. It advised that mobilisations should only proceed if the patient's blood pressure does not fall by $>30 \mathrm{mmHg}$ on sitting up on the edge of the bed, or on getting out of bed

\section{Modifications}

The intervention protocol was not changed during the trial

\section{How well}

Planned: monitoring included time-to-first mobilisation plus number and duration of out-of-bed mobilisation sessions

Actual: the VEM group was noted to have a significantly $(P<0.01)$ reduced time-to-first mobilisation from stroke onset (median 18.5 hours vs 22.4 hours) and increased median number of out-of-bed training sessions per day (6.5 vs 3 ) and increased median amount of out-of-bed training time per day ( 31 vs 10 minutes)

Outcomes mRS at 3 months, evaluation of adverse effects, health-related quality of life, cost effectiveness and cost utility, long-term efficacy, activity limitation, dose response, patient severity, and staff injury

\section{Notes}

Trial ran from 2008 to 2014

\section{Risk of bias}

\begin{tabular}{|c|c|c|}
\hline Bias & Authors' judgement & Support for judgement \\
\hline $\begin{array}{l}\text { Random sequence genera- } \\
\text { tion (selection bias) }\end{array}$ & Low risk & $\begin{array}{l}\text { A remote, web-based, computer-generated randomization procedure was } \\
\text { used. Randomisation was balanced by site and stratified by stroke severity, } \\
\text { based upon the patient's baseline NIHSS score }\end{array}$ \\
\hline $\begin{array}{l}\text { Allocation concealment } \\
\text { (selection bias) }\end{array}$ & Low risk & $\begin{array}{l}\text { All online submissions were secured by use of password and data encryption } \\
\text { procedures. Once participant recruitment data were submitted by the site staff } \\
\text { to the trial website (AVERT Online), the randomization allocation was immedi- } \\
\text { ately provided to the investigator }\end{array}$ \\
\hline $\begin{array}{l}\text { Blinding of participants } \\
\text { and personnel (perfor- } \\
\text { mance bias) } \\
\text { All outcomes }\end{array}$ & Low risk & $\begin{array}{l}\text { Participants were aware that they could be randomized to } 1 \text { of } 2 \text { mobilisation } \\
\text { protocols but were not provided with details. The intervention protocol was } \\
\text { only distributed to trial intervention staff and ethics committees in order to } \\
\text { help maintain blinding and protect against treatment contamination in the tri- } \\
\text { al }\end{array}$ \\
\hline $\begin{array}{l}\text { Blinding of outcome as- } \\
\text { sessment (detection bias) } \\
\text { All outcomes }\end{array}$ & Low risk & $\begin{array}{l}\text { The 3- and 12-month assessments were conducted in person or by telephone } \\
\text { by an assessor who was remote from the location of the acute treatment of the } \\
\text { participant and blind to treatment allocation }\end{array}$ \\
\hline
\end{tabular}

$\begin{array}{ll}\text { Incomplete outcome data } & \text { Low risk } \\ \text { (attrition bias) } & 21 / 2104(1 \%)\end{array}$


AVERT III 2015 (Continued)

All outcomes

Selective reporting (re- Low risk Primary outcome largely complete

porting bias)

Chippala 2015a

\begin{tabular}{ll} 
Methods & Single-blinded RCT with a blinded assessment at the end of follow-up \\
\hline Participants & Acute stroke patients admitted to the stroke unit within 24 hours of symptom onset \\
Both sexes & Able to react to verbal commands \\
& Medical condition stable and physiologically stable (systolic BP 120 to $180 \mathrm{mmHg}$, oxygen saturation $>$ \\
& $92 \%$, heart rate 40 to 100 beats per minute, temperature $\left.<38.5^{\circ} \mathrm{C}\right)$ \\
& 86 participants: 43 intervention, 43 control \\
& Mean age: 60 years \\
& $42 / 80(52 \%)$ men \\
$16 / 80(20 \%)$ haemorrhagic & $27(34 \%)$ had mild stroke (NIHSS 0 to 7$)$ \\
& $42(52 \%)$ moderate stroke (NIHSS 8 to 16$)$ \\
& $11(14 \%)$ severe stroke (NIHSS $>16)$
\end{tabular}

\section{What}

Materials: the trialists "adopted the AVERT Protocol. The intervention group received the following activities: sitting supported in bed, sitting unsupported out of bed, transfer with assistance, roll and sit up, sitting without support, transfer feet on the floor, standing activities, walk-early gait, and advanced gait activities"

\section{Who provided}

Not stated

\section{How}

Not stated

\section{Where}

Provided in the stroke unit

\section{When and how much}

Intervention: "The mobilisation was started (upright and out-of-bed activities) as soon as practical after the recruitment, aiming to have first mobilisation within 24 hours of the onset of the symptoms"

Standard care: "Patients in the standard care group received routine stroke unit care, including the passive and active (if possible) mobilisation, correct positioning in bed, mobilisation in bed, sitting balance activities, facilitation of limb and trunk control activities, education of patient and caregiver. Both groups received standard care treatment, for 45 minutes a day, for seven days or until discharge"

\section{Tailoring}


"The time spent on early and frequent out-of-bed activities was determined by the patient's tolerance ( 5 to 30 minutes), and they received mobilisation for a minimum of two times per day for seven days or until the discharge, whichever was sooner"

\section{Modifications}

Not stated

\section{How well}

Planned: "The mobilisation was started (upright and out-of-bed activities) as soon as practical after the recruitment, which aimed to have first mobilisation within 24 hours of the onset of the symptoms. The time spent on early and frequently out-of-bed activities was determined by the patient's tolerance ( 5 to 30 minutes), and they received mobilisation a minimum of two times per day for seven days, or until the discharge whichever was sooner"

Actual: "time to first mobilization after the symptom onset was a median 18 hours (IQR 16.62 to 19.75 ) in the intervention group and a median 30.5 hours (IQR 29.0 to 35$)$ in the standard care group ( $P<$ 0.001)"

\begin{tabular}{ll}
\hline Outcomes & Primary outcome: Barthel index at the end of 3 months following the ons \\
& Length of hospital stay \\
\hline Notes & Study took place March 2012 to September 2014 \\
& Reported to be a different study and patient group from Chippala 2015b
\end{tabular}

\section{Risk of bias}

\begin{tabular}{lll}
\hline Bias & Authors' judgement & Support for judgement \\
\hline $\begin{array}{l}\text { Random sequence genera- } \\
\text { tion (selection bias) }\end{array}$ & Low risk & $\begin{array}{l}\text { Quote: "randomly allocated equally to either the Intervention group (very ear- } \\
\text { ly mobilization, out of bed within } 24 \text { hours of stroke onset) or the standard } \\
\text { care group, by the computer-generated, randomization procedures" }\end{array}$ \\
\hline $\begin{array}{l}\text { Allocation concealment } \\
\text { (selection bias) }\end{array}$ & Low risk & $\begin{array}{l}\text { Quote: "randomly allocated equally to either the Intervention group (very ear- } \\
\text { ly mobilization out of bed within } 24 \text { hours of stroke onset) or the standard care } \\
\text { group by the computer-generated, randomization procedures, using a con- } \\
\text { cealed opaque envelop method. This randomization list was held by a univer- } \\
\text { sity researcher who was not related to any part of the study" }\end{array}$ \\
\hline
\end{tabular}

Blinding of participants High risk Quote: "Single blind, randomized controlled trial" and personnel (performance bias)

All outcomes

\begin{tabular}{|c|c|c|}
\hline $\begin{array}{l}\text { Blinding of outcome as- } \\
\text { sessment (detection bias) } \\
\text { All outcomes }\end{array}$ & Low risk & $\begin{array}{l}\text { Quote: "The outcome measurements at three-month follow-up were taken di- } \\
\text { rectly by the blinded assessor when the patients visited the hospital for a rou- } \\
\text { tine medical check-up" }\end{array}$ \\
\hline
\end{tabular}
All outcomes 
Chippala 2015b

\begin{tabular}{|c|c|}
\hline Methods & Single blinded, parallel grouped, RCT with a blinded assessment at the end of follow-up \\
\hline \multirow[t]{10}{*}{ Participants } & $\begin{array}{l}\text { Stroke patients admitted to the stroke unit within } 24 \text { hours of symptom onset and physician permission } \\
\text { to mobilise within } 24 \text { hours of stroke }\end{array}$ \\
\hline & Both sexes \\
\hline & Able to react to verbal commands \\
\hline & $\begin{array}{l}\text { Medical condition stable and physiologically stable (systolic BP } 120 \text { to } 180 \mathrm{mmHg} \text {, oxygen saturation }> \\
92 \% \text {, heart rate } 40 \text { to } 100 \text { beats per minute, temperature }<38.5^{\circ} \mathrm{C} \text { ) }\end{array}$ \\
\hline & Mean age: 63.3 years \\
\hline & 54 participants: 27 intervention, 27 control \\
\hline & $28 / 49(57 \%)$ men \\
\hline & $11 / 49(22 \%)$ haemorrhagic \\
\hline & $27 / 48(56 \%)$ had mild stroke (NIHSS 0 to 7 ) \\
\hline & $21 / 48(44 \%)$ moderate stroke (NIHSS 8 to 16 ) \\
\hline
\end{tabular}

Both the groups received standard care treatment including routine stroke unit care, for 45 minutes a day, for seven days, or until discharge

The intervention group received VEM mobilisation activities, initiated within 24 hours of the stroke onset, in addition to the standard care. They performed early and frequent out-of-bed activities including sitting, standing, walking. The duration of mobilisation was determined by the participant's tolerance ( 5 to 30 minutes) with a frequency of a minimum of 2 times per day for 7 days or until the discharge, whichever was sooner

The mRS at 3 months. A good outcome was defined as mRS of 0 to 2
Barthel Index at 3 months
Adverse events during first 3 months
Length of hospital stay (days)

Notes Study took place from September 2014 to June 2015

Reported to be a different study and patient group from Chippala 2015a

\section{Risk of bias}

\begin{tabular}{|c|c|c|}
\hline Bias & Authors' judgement & Support for judgement \\
\hline $\begin{array}{l}\text { Random sequence genera- } \\
\text { tion (selection bias) }\end{array}$ & Unclear risk & $\begin{array}{l}\text { Quote: "single blinded, parallel grouped, randomized controlled trial with a } \\
\text { blinded assessment at the end of follow-up" }\end{array}$ \\
\hline $\begin{array}{l}\text { Allocation concealment } \\
\text { (selection bias) }\end{array}$ & Low risk & $\begin{array}{l}\text { Quote: "randomization procedures using a concealed opaque envelop } \\
\text { method" }\end{array}$ \\
\hline $\begin{array}{l}\text { Blinding of participants } \\
\text { and personnel (perfor- } \\
\text { mance bias) } \\
\text { All outcomes }\end{array}$ & High risk & $\begin{array}{l}\text { Quote: "single blinded, parallel grouped, randomized controlled trial with a } \\
\text { blinded assessment at the end of follow-up" }\end{array}$ \\
\hline $\begin{array}{l}\text { Blinding of outcome as- } \\
\text { sessment (detection bias) }\end{array}$ & Low risk & $\begin{array}{l}\text { Quote: "single blinded, parallel grouped, randomized controlled trial with a } \\
\text { blinded assessment at the end of follow-up" }\end{array}$ \\
\hline
\end{tabular}


Chippala 2015b (Continued)

All outcomes

Incomplete outcome data Low risk $\quad 5 / 54(9 \%)$ missing at follow-up
(attrition bias)
All outcomes

\begin{tabular}{l}
$\begin{array}{l}\text { Selective reporting (re- } \\
\text { porting bias) }\end{array}$ \\
\hline
\end{tabular}

\section{Langhorne 2010}

$\begin{array}{ll}\text { Methods } & \text { A pilot randomized trial (using a } 2 \times 2 \text { factorial design) to compare conventional stroke unit procedures } \\ \text { with more progressive (nurse-led) protocols of early mobilisation (EM), automated physiological moni- } \\ \text { toring, or both } \\ \text { As there was no statistical interaction between the } 2 \text { protocols, the EM arms were compared directly } \\ \text { with the normal mobilisation arms } \\ \text { 1 stroke unit in Glasgow, Scotland } \\ \text { Recruited } 32 \text { acute stroke patients admitted to hospital within } 24 \text { hours (and recruited within } 36 \text { hours } \\ \text { of symptom onset) with no premorbid severe disability } \\ \text { Of the } 16 \text { EM patients, } 8 \text { were allocated to the EM protocol alone and } 8 \text { to the EM plus automated moni- } \\ \text { toring protocols } \\ \text { Of the } 16 \text { controls, } 8 \text { were allocated to normal mobilisation alone and } 8 \text { to normal mobilisation plus the } \\ \text { automated monitoring protocol } \\ \text { Mean age: } 67.5 \text { years } \\ 16 / 32(50 \%) \text { men } \\ 1 / 32(3 \%) \text { haemorrhagic } \\ \text { Mean NIHSS } 5 \\ 23(72 \%) \text { had mild stroke (NIHSS } 1 \text { to } 7) \\ 7(22 \%) \text { moderate stroke (NIHSS } 8 \text { to } 16) \\ 2(6 \%) \text { severe stroke (NIHSS }>16)\end{array}$

\section{What}

Materials: protocols for early mobilisation (VEM) or intensive physiological monitoring, or both, or standard care alone (control). VEM was planned to be similar to AVERT protocol but did not have access to the same written protocol.

Procedures: the VEM protocol aimed to get participants up to sit, stand, and walk within 24 hours of the stroke and continue this at least 4 times per day

Controls: the stroke unit had a philosophy of getting patients up to sit, stand, and walk early but did not have staff specifically allocated to this role. Mobilisation was normally provided by physiotherapists and nurses (30 to 60 minutes per day). Normal monitoring involved intermittent (4-hourly) checking of pulse, temperature, oxygen saturation, and blood pressure

\section{Who provided}

The research nurse had a role ensuring the VEM protocol was implemented in conjunction with physiotherapy and nursing staff

\section{How}


The research nurse facilitated the VEM protocol that was largely delivered face-to-face by physiotherapy and nursing staff

Where

Provided in the stroke unit

\section{When and how much}

For 1 week after recruitment or until discharge

Tailoring

EM could be adapted according to the abilities of the participant

\section{Modifications}

Mobilisation could be halted if monitoring of pulse, temperature, oxygen saturation, or blood pressure suggested abnormal changes

\section{How well}

Planned: the EM protocol aimed to get participants up to sit, stand, and walk within 24 hours of the stroke and continue this at least 4 times per day. This was monitored as: 1) time-to-first mobilisation (attempt to get the participant out of bed, to sit, stand, or walk); 2) best level of mobilisation activity achieved (lying, sitting, standing, walking); and 3) participant activity (using automated activity monitor recordings)

Actual: median time from stroke to first mobilisation in the VEM group was 27.3 hours (IQR 26.0 to 29.0) vs 32.0 hours (IQR 22.5 to 47.3 ) in controls $(P=0.31)$, however a significantly greater number of EM participants $(P=0.03)$ were mobilised within 1 hour of randomization. A significantly great number of EM participants $(P=0.02)$ achieved standing or walking when mobilisation was recorded using activity monitors

\section{Outcomes}

$\mathrm{mRS}$ at 3 months, adverse events, patient activity, neurological deterioration, Barthel Index at 1 week and 3 months, walking speed ( 1 week and discharge), patient satisfaction, resource allocation

Notes Recruitment took place in February 2007 to January 2008

\section{Risk of bias}

\begin{tabular}{|c|c|c|}
\hline Bias & Authors' judgement & Support for judgement \\
\hline $\begin{array}{l}\text { Random sequence genera- } \\
\text { tion (selection bias) }\end{array}$ & Low risk & $\begin{array}{l}\text { The randomization sequence was to } 1 \text { of } 4 \text { nurse-led treatment protocols ( } 2 \text { of } \\
\text { which delivered EM). The sequence was computer-generated in blocks of } 4\end{array}$ \\
\hline $\begin{array}{l}\text { Allocation concealment } \\
\text { (selection bias) }\end{array}$ & Low risk & $\begin{array}{l}\text { Quote: "patients were then randomly allocated by telephoning a secretary in } \\
\text { an independent office who logged the patient and opened the next in a series } \\
\text { of sequentially numbered opaque sealed envelopes" }\end{array}$ \\
\hline $\begin{array}{l}\text { Blinding of participants } \\
\text { and personnel (perfor- } \\
\text { mance bias) } \\
\text { All outcomes }\end{array}$ & Unclear risk & $\begin{array}{l}\text { Participants were aware that they were being randomized to different care } \\
\text { protocols. No specific measures were in place to segregate participants but } \\
\text { recruitment did not result in participants receiving different protocols in the } \\
\text { same ward space }\end{array}$ \\
\hline
\end{tabular}

\begin{tabular}{|c|c|c|}
\hline $\begin{array}{l}\text { Blinding of outcome as- } \\
\text { sessment (detection bias) }\end{array}$ & Low risk & $\begin{array}{l}\text { Key outcome observations were blinded to treatment allocation by using an } \\
\text { independent assessor at day } 5 \text { and at } 3 \text { months }\end{array}$ \\
\hline
\end{tabular}

Incomplete outcome data Low risk No losses after randomization
(attrition bias)


Langhorne 2010 (Continued)

All outcomes

Selective reporting (re- Low risk $\quad$ Primary outcome complete
porting bias)

\section{Morreale 2016}

\begin{tabular}{|c|c|}
\hline Methods & Multicenter, factorial, single blind RCT \\
\hline \multirow[t]{9}{*}{ Participants } & First ischaemic stroke (middle cerebral artery) 6 to 24 hours from onset \\
\hline & $\begin{array}{l}\text { Exclusion criteria included: mild stroke (NIHSS }<2 \text { ), MMSE }<26 \text {, neurological or cardiovascular instabili- } \\
\text { ty, significant comorbidity }\end{array}$ \\
\hline & 340 participants: 220 intervention, 120 control \\
\hline & Mean age: 64 years \\
\hline & $246 / 340(72 \%)$ men \\
\hline & $0 / 340(0 \%)$ haemorrhagic \\
\hline & $37(11 \%)$ had mild stroke (NIHSS 3 to 6$)$ \\
\hline & $260(76 \%)$ moderate stroke (NIHSS 7 to 14$)$ \\
\hline & $43(13 \%)$ severe stroke (NIHSS > 14) \\
\hline
\end{tabular}

\section{What}

A factorial design was used to compare early proprioceptive neuromuscular facilitation (PNF) or cognitive therapeutic exercise (CTE) commenced within 24 hours of admission, with delayed PNF and CTE groups, where treatment started 4 days later

Materials: all physiotherapists were specifically trained for treatment protocols.

Procedures: early rehabilitation: daily out-of-bed activity with either PNF or CTE commenced within 24 hours

Usual care: routine hospital care for first 4 days, followed by either PNF or CTE

\section{Who provided}

Physiotherapists

\section{How}

Physiotherapists were specifically trained for treatment protocols and were not aware of the clinical features and study objective

\section{Where}

Neurology department of 2 stroke centres in Rome

\section{When and how much}

Early rehabilitation: daily out-of-bed activity (with either PNF or CTE). Dose set as 1 hour/day for first 4 days; followed by 2.25 hours/day, daily for 14 weeks; followed by 1.5 hours/day, 5 days/week until final medical follow-up (mean of 38 weeks)

Usual care: routine hospital care for first 4 days, followed by either PNF or CTE. Dose based on standard hospital care for first 4 days, and (from day 5) as per early rehabilitation groups

\section{Tailoring}


Morreale 2016 (Continued)

Not stated

\section{Modifications}

Not stated

\section{How well}

Planned: a physical therapists' co-ordinator monitored the adherence to and homogeneity of treatment protocols in all settings

Actual: not reported

Primary outcome measures were disability (mRS and Barthel Index)
Secondary outcomes:
- safety - immobility-related adverse events at 3 and 12 months.
- Six Minute Walking Test
- Motricity index
- MMSE

Notes

Trial ran between January 2008 and January 2013

Loss to follow-up was not divided by group

3 months: 13 dead, 25 'lack of compliance'

12 months: 16 dead, 27 'lack of compliance', 4 recurrent stroke

\section{Risk of bias}

\begin{tabular}{|c|c|c|}
\hline Bias & Authors' judgement & Support for judgement \\
\hline $\begin{array}{l}\text { Random sequence genera- } \\
\text { tion (selection bias) }\end{array}$ & Low risk & $\begin{array}{l}\text { Computer-generated randomization sequence in blocks of } 4 \text { to } 1 \text {. A factorial } \\
\text { design was used to compare early proprioceptive neuromuscular facilitation } \\
\text { (PNF) or cognitive therapeutic exercise (CTE) commenced within } 24 \text { hours of } \\
\text { admission, with delayed PNF and CTE groups, where treatment started } 4 \text { days } \\
\text { later } \\
\text { Randomisation was stratified by age, sex, risk factors, stroke aetiology, side, } \\
\text { NIHSS score }\end{array}$ \\
\hline $\begin{array}{l}\text { Allocation concealment } \\
\text { (selection bias) }\end{array}$ & Unclear risk & Quote: "..physical therapists' coordinator randomly assigned all patients..." \\
\hline $\begin{array}{l}\text { Blinding of participants } \\
\text { and personnel (perfor- } \\
\text { mance bias) } \\
\text { All outcomes }\end{array}$ & High risk & $\begin{array}{l}\text { Quote: "All physiotherapists were specifically trained for treatment protocols } \\
\text { and were unaware of clinical features....and study objective" }\end{array}$ \\
\hline $\begin{array}{l}\text { Blinding of outcome as- } \\
\text { sessment (detection bias) } \\
\text { All outcomes }\end{array}$ & Low risk & $\begin{array}{l}\text { All scales at } 3 \text { and } 12 \text { months were administered by "two different neurologists } \\
\text { who were blinded for treatment". }\end{array}$ \\
\hline $\begin{array}{l}\text { Incomplete outcome data } \\
\text { (attrition bias) } \\
\text { All outcomes }\end{array}$ & Unclear risk & $\begin{array}{l}25 / 340(7 \%) \text { of participants were lost to follow-up at } 3 \text { months and } 27 / 340(8 \%) \\
\text { at } 12 \text { months due to 'lack of compliance' }\end{array}$ \\
\hline
\end{tabular}


Morreale 2016 (Continued)

Selective reporting (re- Unclear risk Primary outcome largely complete porting bias)

Morreale 2016 CTE

Methods Cognitive therapeutic exercise (CTE) subgroup of Morreale 2016

\section{Participants}

Interventions

\section{Outcomes}

\section{Notes}

\section{Risk of bias}

\begin{tabular}{lll}
\hline Bias & Authors' judgement & Support for judgement \\
\hline $\begin{array}{l}\text { Random sequence genera- } \\
\text { tion (selection bias) }\end{array}$ & Low risk & See Morreale 2016 \\
\hline $\begin{array}{l}\text { Allocation concealment } \\
\text { (selection bias) }\end{array}$ & Unclear risk & See Morreale 2016 \\
\hline $\begin{array}{l}\text { Blinding of participants } \\
\text { and personnel (perfor- } \\
\text { mance bias) }\end{array}$ & Unclear risk & See Morreale 2016 \\
All outcomes & \\
\hline $\begin{array}{l}\text { Blinding of outcome as- } \\
\text { sessment (detection bias) } \\
\text { All outcomes }\end{array}$ & Low risk & See Morreale 2016 \\
\hline $\begin{array}{l}\text { Incomplete outcome data } \\
\text { (attrition bias) } \\
\text { All outcomes }\end{array}$ & Unclear risk & See Morreale 2016 \\
\hline $\begin{array}{l}\text { Selective reporting (re- } \\
\text { porting bias) }\end{array}$ & Unclear risk & See Morreale 2016 \\
\hline
\end{tabular}

\section{Morreale 2016 PNF}

Methods Neuromuscular facilitation (PNF) subgroup of Morreale 2016

\section{Participants}

$$
\text { Interventions }
$$

\section{Outcomes}

\section{Notes}


Morreale 2016 PNF (Continued)

Risk of bias

\begin{tabular}{|c|c|c|}
\hline Bias & Authors' judgement & Support for judgement \\
\hline $\begin{array}{l}\text { Random sequence genera- } \\
\text { tion (selection bias) }\end{array}$ & Low risk & See Morreale 2016 \\
\hline $\begin{array}{l}\text { Allocation concealment } \\
\text { (selection bias) }\end{array}$ & Unclear risk & See Morreale 2016 \\
\hline $\begin{array}{l}\text { Blinding of participants } \\
\text { and personnel (perfor- } \\
\text { mance bias) } \\
\text { All outcomes }\end{array}$ & Unclear risk & See Morreale 2016 \\
\hline $\begin{array}{l}\text { Blinding of outcome as- } \\
\text { sessment (detection bias) } \\
\text { All outcomes }\end{array}$ & Low risk & See Morreale 2016 \\
\hline $\begin{array}{l}\text { Incomplete outcome data } \\
\text { (attrition bias) } \\
\text { All outcomes }\end{array}$ & Unclear risk & See Morreale 2016 \\
\hline $\begin{array}{l}\text { Selective reporting (re- } \\
\text { porting bias) }\end{array}$ & Unclear risk & See Morreale 2016 \\
\hline
\end{tabular}

Poletto 2015

Methods Quote: "randomized, controlled, single-blind clinical trial compared early mobilization (within $48 \mathrm{~h}$ of symptom onset) with routine physical therapy"

Participants Quote: "adult patients with CT- or MRI-confirmed ischemic stroke within $48 \mathrm{~h}$ of symptom onset who were admitted on weekdays from March to November 2012 to the acute vascular unit or general emergency unit of a large urban emergency department"

39 participants: 19 intervention, 20 control

Mean age: 65 years

$13 / 37$ (35\%) men

$0 / 37(0 \%)$ haemorrhagic

Mean NIHSS score of 10.5

$11(30 \%)$ had mild stroke (NIHSS 0 to 5$)$

$12(32 \%)$ moderate stroke (NIHSS 6 to 11$)$

$14(38 \%)$ severe stroke (NIHSS > 11)

\section{What}

Materials: unclear for staff but "patients and their families received a manual developed for the study, with guidance on positioning in bed and posture shifting to use at home after discharge"

Procedures: these "focused on getting out of bed, sitting in a chair, or standing (whenever and as soon as possible), and conducting functional training and motor relearning, pursuant to the Bobath concept. Exercises were performed bilaterally with at least 5 repetitions for each joint and each exercise, with emphasis on deficits on the impaired side. In addition, patients and their families received a manual 
developed for the study, with guidance on positioning in bed and posture shifting to use at home after discharge. Intervention patients were mobilized 5 times a week, once a day, for approximately $30 \mathrm{~min}$ per session, in addition to sitting out of bed for at least 30 min whenever possible"

Controls: they received "conventional physical therapy performed when requested by the staff. Therapy varied according to the patients' needs and the availability of physical therapists but generally included global motor exercises and respiratory therapy (ordinarily in bed). The duration of standard-care therapy sessions was approximately $15 \mathrm{~min} "$

\section{Who provided}

Quote: "The program was carried out by trained physical therapists"

\section{How}

Quote: "The program was carried out by trained physical therapists"

\section{Where}

The acute vascular unit or general emergency unit of a large urban hospital in Brazil.

\section{When and how much}

Quote: "Exercises were performed bilaterally with at least 5 repetitions for each joint and each exercise, and emphasis on deficits on the impaired side. Intervention patients were mobilized 5 times a week, once a day, for approximately $30 \mathrm{~min}$ per session, in addition to sitting out of bed for at least $30 \mathrm{~min}$ whenever possible". "Sessions were held until hospital discharge or the 14th treatment day, whichever occurred first, regardless of where the patient was located"

\section{Tailoring}

Not stated

\section{Modifications}

Not stated

\section{How well}

Planned: "The session duration (in minutes) and number of sessions were recorded. Sessions were held until hospital discharge or the 14th treatment day, whichever occurred first, regardless of where the patient was located"

Actual: "Intervention patients received mobilization earlier and more frequently than controls (table 2). The median time from stroke to first mobilization was $43 \mathrm{~h}$ (vs. $72 \mathrm{~h}$ in the CG), and the total duration of mobilization during the hospitalization period was $135 \mathrm{~min}$ (IQR 85 to 213; vs. 0 min in the CG (IQR 0 to 50)). Only 2 patients did not initiate early mobilization (within $48 \mathrm{~h}$ ) in the IG. Moreover, only 5 patients in the CG $(26 \%)$ received physical therapy during hospitalization, with an average duration of $15 \mathrm{~min}$ per session. After hospital discharge, $57 \%$ of the patients in the IG and $37 \%$ in the CG underwent physical therapy sessions $(P=0.28)$. IG patients had more out-of-bed activities compared with controls ( 4.3 vs 0.3 ), initiating activities while still in the ED. Only the 5 patients who received physical therapy in the CG left their beds; all other controls remained bedbound during hospitalization"

Outcomes The trialists "sought only to evaluate the feasibility and safety of the intervention. The primary outcome measures were functional capacity (mRS score 0 to 2 ) and mortality at 3 months.

Feasibility endpoints were (a) time-to-first mobilisation, and (b) total duration of motor physical therapy.

Safety endpoints were: (a) complications during early mobilisation (first $48 \mathrm{~h}$ ), i.e. symptomatic hypotension (syncope or presyncope) or neurological deterioration (defined as any worsening in NIHSS score); (b) falls during hospitalisation and within 3 months of stroke; (c) complications related to immobility (pneumonia, pulmonary embolism, and deep vein thrombosis) at 3 months, and (d) death within 3 months. 
Poletto 2015 (Continued)

Secondary outcomes were measured at 3 months (mRS score 0 to 1 , mRS score 0 to 2 , mean NIHSS score, and $\mathrm{mBI} \geq 85$ )".

\begin{tabular}{ll}
\hline Notes & ClinicalTrials.gov NCT01694992 \\
& $\begin{array}{l}\text { Reported as a pilot RCT that recruited from March to November 2012. Planned recruitment was for } 174 \\
\text { participants (82 per group) but was limited by slow recruitment }\end{array}$
\end{tabular}

\section{Risk of bias}

\begin{tabular}{|c|c|c|}
\hline Bias & Authors' judgement & Support for judgement \\
\hline $\begin{array}{l}\text { Random sequence genera- } \\
\text { tion (selection bias) }\end{array}$ & Low risk & $\begin{array}{l}\text { Quote: "Randomization was performed online (randomization.com) by a study } \\
\text { investigator, using a randomization plan stratified by blocks of varying sizes } \\
\text { (blocks of } 2,4, \text { or } 6 \text { )" }\end{array}$ \\
\hline $\begin{array}{l}\text { Allocation concealment } \\
\text { (selection bias) }\end{array}$ & Low risk & $\begin{array}{l}\text { Quote: "randomly allocated to an intervention group (IG) or a control group } \\
\text { (CG), with the allocation records stored in opaque, sealed, and sequentially } \\
\text { numbered envelopes" }\end{array}$ \\
\hline $\begin{array}{l}\text { Blinding of participants } \\
\text { and personnel (perfor- } \\
\text { mance bias) } \\
\text { All outcomes }\end{array}$ & High risk & $\begin{array}{l}\text { Quote: "Patients were informed that they would begin physical therapy on the } \\
\text { first day of assessment (IG) or follow the hospital routine (CG)" }\end{array}$ \\
\hline $\begin{array}{l}\text { Blinding of outcome as- } \\
\text { sessment (detection bias) } \\
\text { All outcomes }\end{array}$ & Low risk & $\begin{array}{l}\text { Quote: "Baseline and postintervention measures were performed by a study } \\
\text { investigator who remained blinded to group allocation. To ensure blinding of } \\
\text { any monitoring neurologists, no notes of group allocation were made in the } \\
\text { hospital's electronic medical record". }\end{array}$ \\
\hline $\begin{array}{l}\text { Incomplete outcome data } \\
\text { (attrition bias) } \\
\text { All outcomes }\end{array}$ & High risk & $6 / 39(15 \%)$ missing at 3 month follow-up \\
\hline $\begin{array}{l}\text { Selective reporting (re- } \\
\text { porting bias) }\end{array}$ & Low risk & Primary outcome largely complete \\
\hline
\end{tabular}

\section{SEVEL 2016}

\begin{tabular}{|c|c|}
\hline Methods & Prospective multicenter RCT testing 2 sitting procedures at the acute phase of ischaemic stroke \\
\hline \multirow[t]{8}{*}{ Participants } & Acute ischaemic stroke patients recruited from 11 centres in the North West region of France \\
\hline & $\begin{array}{l}\text { Participants were: over } 18 \text { years age, had neurological deficits, haemorrhage excluded on imaging, en- } \\
\text { rolled in a healthcare plan (French social security) }\end{array}$ \\
\hline & Exclusions included; very mild (NIHSS $<3$ ) or very severe stroke $($ NIHSS $>22)$, reduced consciousness \\
\hline & $\begin{array}{l}\text { Glasgow Coma Score < 13), fluctuating neurological signs (history of worsening linked to an upright po- } \\
\text { sitioning), known symptomatic intra-cranial stenosis }>50 \% \text {, vomiting or difficulty in breathing, con- } \\
\text { traindication for sitting, e.g. deep vein thrombosis or lower limb fracture, prior dependency Rankin } \\
\text { score } 3 \text { to } 6 \text {, anticipated difficulty in follow-up }\end{array}$ \\
\hline & 167 participants: 82 intervention, 85 control \\
\hline & Mean age: 70 years \\
\hline & $89 / 138(64 \%)$ men \\
\hline & $0 / 138(0 \%)$ haemorrhagic \\
\hline
\end{tabular}


SEVEL 2016 (Continued)

\author{
Mean NIHSS 7.5 \\ 76 (56\%) had mild stroke (Rankin score 0 to 3 ) \\ 45 (34\%) moderate stroke (Rankin score 4) \\ $13(10 \%)$ severe stroke (Rankin score 5)
}

\title{
What
}

This study aimed to test 2 different protocols for sitting in acute ischaemic stroke patients.

Early protocol: participants seated out of bed at the earliest time possible, ideally the day of stroke onset (day 0 ) and no later than the calendar day after stroke onset

Progressive protocol: the participant would be positioned in bed at $30^{\circ}, 45^{\circ}$ the day after (day 1 ), and $60^{\circ}$ at day 2 , and sitting out of bed at day 3 (which corresponds to the first sitting in this group). Those angles reflect the position of the upper body relative to the bed (and floor)

\section{Who provided}

The physiotherapist or the nurses were in charge of collecting the data (e.g. blood pressure, tolerance) related to the protocol

\section{How}

For both protocols, minimal duration of the first sitting was 15 minutes. The procedure could be continued, depending on participant fatigue and tolerance (60 minutes maximum). The physiotherapist or the nurses were in charge of collecting the data (blood pressure, tolerance. . .) related to it. Sitting posture (legs dangling or feet positioned on a foot rest), was done as usual, in keeping with each unit's protocol. The use of a lifter, when necessary, was allowed

\section{Where}

Stroke centres of 11 hospitals in North West France

\section{When and how much}

For both protocols, minimal duration of the first sitting was 15 minutes. The procedure could be continued, depending on patient fatigue and tolerance ( 60 minutes maximum). Sitting was repeated on a daily basis according to initial tolerance of the procedure, as approved by the physician in charge

\section{Tailoring}

Close monitoring of blood pressure and heart rate was performed: before the sitting procedure, immediately after, and 5 minutes after. While sitting, participants showing any sign of low tolerance, defined by neurological worsening (of current or new neurological deficits), vagal reaction (bradycardia or nausea), a greater than $40 \mathrm{mmHg}$ increase of blood pressure topping $180 / 100 \mathrm{mmHg}$, or a symptomatic decrease in blood pressure, would be put back in bed. Sitting was repeated on a daily basis according to initial tolerance of the procedure, as approved by the physician in charge

\section{Modifications}

While sitting, participants showing any sign of low tolerance, defined by neurological worsening (of current or new neurological deficits), vagal reaction (bradycardia or nausea), a greater than $40 \mathrm{mmHg}$ increase of blood pressure topping $180 / 100 \mathrm{mmHg}$, or a symptomatic decrease in blood pressure, would be put back in bed

\section{How well}

Planned: the early protocol intended that participants would be seated, out of bed, at the earliest time possible, no later than the calendar day after stroke onset. In the progressive protocol, the participant would be positioned in bed at $30^{\circ}, 45^{\circ}$ the day after (day 1 ), and $60^{\circ}$ at day 2 , and sitting out of bed at day 3 (which corresponds to the first sitting in this group) 
Actual: time from stroke to the first sitting time was $1.1 \pm 0.2$ days in the early sitting group versus $3 \pm$ 0.2 days in the progressive group

First sitting lasted significantly longer in the progressive group compared to the early group: $83.7 \pm 94.7$ minutes versus $56.6 \pm 41.7$ minutes respectively $(P<0.05)$. Tolerance of the sitting procedure was the same in the early and progressive sitting groups, with a prevalence of side effects of $14.5 \%$ and $13.7 \%$, respectively. Sitting was continued daily for both groups during hospitalisation in $96 \%$ of cases.

NB: a small amount of out-of-bed activity was incorporated in transferring between bed and chair, which started 2 days earlier in the intervention group than the standard care group

Physiotherapy and deep vein thrombosis prevention by low molecular weight heparin were performed as usual in each unit

\section{Outcomes}

The primary outcome measure was the proportion of $\mathrm{mRS} 0$ to 2 at 3 months visit after stroke onset

Secondary outcomes were assessed at 7 days (or the day of discharge, if before 7 days), and the 3month follow-up and included:

- NIHSS score, Rankin score, Barthel score;

- data about the tolerance of the sitting positioning (including prevalence of side effects that forced termination of the procedure);

- length of hospitalisation;

- complications that occurred during hospital stay were reviewed at 3 months using a multiple-choice list, and based on both participant interview and medical records;

- duration of sitting out of bed was calculated from the recorded time at which the participant was positioned seated out of bed to the time at which the participant would be put back in bed

Notes

SEVEL (Stroke and Early VErticaL positioning) study

clinicaltrials.org registration number NCT01573299

The enrolment period covered November 2011 to April 2014. The study ended prematurely (after 167 of a target of 400) as it became unviable, due to the slow recruitment rate

\section{Risk of bias}

\begin{tabular}{lll}
\hline Bias & Authors' judgement & Support for judgement \\
\hline $\begin{array}{l}\text { Random sequence genera- } \\
\text { tion (selection bias) }\end{array}$ & Low risk & $\begin{array}{l}\text { Quote: "The random sequence was generated by our statistician (CV) using the } \\
\text { SAS software" }\end{array}$ \\
\hline $\begin{array}{l}\text { Allocation concealment } \\
\text { (selection bias) }\end{array}$ & Low risk & $\begin{array}{l}\text { Randomisation between the early and progressive sitting groups was per- } \\
\text { formed via "numbered sealed envelopes that the investigator would draw } \\
\text { from, in consecutive fashion (with blocks of } 4 \text { in 1:1 ratio, stratified by center) } \\
\text { each time a patient was enrolled in the study" }\end{array}$ \\
\hline $\begin{array}{l}\text { Blinding of participants } \\
\text { and personnel (perfor- } \\
\text { mance bias) }\end{array}$ & Unclear risk & Quote: "Data were reported online using a server dedicated to the study" \\
$\begin{array}{l}\text { All outcomes } \\
\end{array}$ &
\end{tabular}

\begin{tabular}{lll}
\hline $\begin{array}{l}\text { Blinding of outcome as- } \\
\text { sessment (detection bias) }\end{array}$ & High risk & $\begin{array}{l}\text { Quote: "Evaluations were made during the intermediate time point at } 7 \text { days } \\
\text { (or the day of discharge, if before } 7 \text { days) and at } 3 \text { months after stroke, by a } \\
\text { All outcomes }\end{array}$ \\
& $\begin{array}{l}\text { neurologist from the same stroke unit, aware of the study and unblinded to the } \\
\text { patient group assignment" }\end{array}$
\end{tabular}

Incomplete outcome data High risk Substantial drop out $(29 / 167 ; 17 \%)$ after randomization
(attrition bias) 
SEVEL 2016 (Continued)

All outcomes
Participants with major deviation to the protocol or serious adverse event that were enrolled but could not continue the study were assigned a Rankin score in the category 3 to 6

Selective reporting (re-

Unclear risk

Primary outcome largely complete

porting bias)

Sundseth 2012

Methods Prospective RCT with blinded assessment at follow-up

Participants

1 stroke unit in Akershus, Norway

Acute stroke patients (infarct or haemorrhage), defined according to the WHO criteria, admitted to the stroke unit within 24 hours of stroke

Exclusion criteria included; age < 18 years, prior dependency (mRS score 1 to 5), secondary haemorrhage, acute coronary disease, treated with thrombolysis or thrombectomy

65 participants recruited (12 lost to follow-up): 32 intervention ( 7 lost after randomization), 33 control (5 lost after randomization)

Mean age: 77 years

25/56 (45\%) men

$5 / 56(18 \%)$ haemorrhagic

Mean NIHSS 8

$37(66 \%)$ had mild stroke (NIHSS 1 to 7$)$

$11(20 \%)$ moderate stroke (NIHSS 8 to 16$)$

$8(14 \%)$ severe stroke $(\mathrm{NIHSS}>16)$

Significantly lower prevalence of diabetes in the intervention group: $2 / 27(7 \%)$ vs $8 / 29(28 \%)$; $P=0.05$

Interventions

\section{What}

Materials: both groups received standard stroke unit care. No detailed mobilisation protocol was used.

Procedures: VEM participants were mobilised out of bed as soon as possible after randomization and at least 24 hours from admission to hospital. Mobilisation, meaning all out-of-bed activities, was carried out several times per day. Control participants started mobilisation 24 to 48 hours from admission

\section{Who provided}

VEM was performed by physiotherapists, nursing staff, and occupational therapists

\section{How}

VEM participants were mobilised out of bed as soon as possible after randomization and at least 24 hours from admission to hospital by physiotherapy, nursing, and occupational therapy staff

\section{Where}

In the stroke unit

\section{When and how much}

Until discharge from the stroke unit

\section{Tailoring}


All mobilisation was adjusted to the participant's needs and abilities. A neurologist could be called to postpone mobilisation in participants with deteriorating condition while exercising

\section{Modifications}

The intervention protocol was not changed during the trial. The type and amount of mobilisation was the same for infarct and haemorrhage

\section{How well}

Planned: the type and amount of mobilisation was similar to the VEM group but neither time nor duration was recorded

Actual: 5/32 VEM participants were not mobilised to protocol ( 3 were mobilised within 48 hours and 2 within 72 hours)

$1 / 33$ control participant had very delayed mobilisation at 85 hours. Median time from stroke to first mobilisation in the VEM group was 13.1 hours (IQR 8.5 to 25.6 ) vs 33.3 hours (IQR 26.0 to 39.0 ) in controls (P $=0.001)$. Neither frequency nor duration of mobilisation was recorded

Outcomes were recorded at discharge and 3 months and included:
- good outcome (mRS of 0 to 2);
- survival;
- ADL score (Barthel index);
- Complications were classified as:
$*$ stroke-related (recurrent stroke or intracerebral haemorrhage, transient ischaemic attack, post-
* immobility-related (deep vein thrombosis, pulmonary embolism, bedsores, pneumonia, urinary
tract infection, and falls); and comorbidity-related (angina pectoris, myocardial infarction)
$*$ Hospital Anxiety and Depression Scale (HADS)

Notes

Study recruited during 2007 and 2009 to 2010. Additional activity was not specified, but the intervention group participants appeared to have received more out-of-bed activity in total, because it commenced earlier. Study was powered to include 246 participants, but stopped early because of slow recruitment

9 participants were excluded after randomization when the diagnosis of stroke could not be confirmed

\section{Risk of bias}

\begin{tabular}{|c|c|c|}
\hline Bias & Authors' judgement & Support for judgement \\
\hline $\begin{array}{l}\text { Random sequence genera- } \\
\text { tion (selection bias) }\end{array}$ & Low risk & Quote: "computer-generated, blocked, randomization procedures" \\
\hline $\begin{array}{l}\text { Allocation concealment } \\
\text { (selection bias) }\end{array}$ & Low risk & Quote: "using opaque envelopes" \\
\hline $\begin{array}{l}\text { Blinding of participants } \\
\text { and personnel (perfor- } \\
\text { mance bias) } \\
\text { All outcomes }\end{array}$ & Unclear risk & No specific comment \\
\hline $\begin{array}{l}\text { Blinding of outcome as- } \\
\text { sessment (detection bias) } \\
\text { All outcomes }\end{array}$ & Low risk & $\begin{array}{l}\text { Quote: "blinded assessment at the end of follow-up". Blinding was not present } \\
\text { for earlier outcomes }\end{array}$ \\
\hline $\begin{array}{l}\text { Incomplete outcome data } \\
\text { (attrition bias) }\end{array}$ & Unclear risk & A total of $12 / 65(18 \%)$ patients randomized did not undergo 3-month follow-up \\
\hline
\end{tabular}


Sundseth 2012 (Continued)

All outcomes
Selective reporting (re-
Unclear risk
Primary outcome largely complete

porting bias)

ADL: activities of daily living

BP: blood pressure

ICU: intensive care unit

IQR: interquartile range

MMSE: Mini Mental State Examinsation

mRS: modified Rankin scale

NIHSS: National Institutes of Health Stroke Scale

$\mathrm{RCT}$ : randomized controlled trial

rtPA: recombinant tissue plasminogen activator

VEM: very early mobilisation

Characteristics of excluded studies [ordered by study ID]

\begin{tabular}{|c|c|}
\hline Study & Reason for exclusion \\
\hline AMOBES 2017 & Commenced mobilisation after 48 hours \\
\hline Asberg 1989 & Insufficient details to confirm eligibility \\
\hline Chu 2003 & Timing not stated \\
\hline Di Lauro 2003 & No time difference between groups \\
\hline Diserens 2010 & Commenced mobilisation after 52 hours \\
\hline Duan 2006 & Timing not stated, additional psychotherapy and cognitive therapy \\
\hline Fang 2001a & Rehabilitation started 0-7 days after onset \\
\hline Fang 2001b & Rehabilitation started within 7 days of onset \\
\hline Gong 2003 & Timing not stated, not randomised \\
\hline Gorbunov 2003 & Insufficient details to confirm eligibility \\
\hline Gu 2006 & Timing not stated \\
\hline Guan 2001 & Timing not stated, confounded by additional psychotherapy and swallowing therapy \\
\hline Hamrin 1982 & $\begin{array}{l}\text { Confounded by rehabilitation package (a large number of components of stroke unit care versus } \\
\text { none), pseudo-RCT }\end{array}$ \\
\hline Hara 2001 & Insufficient details to confirm eligibility \\
\hline Huang 2001 & Timing not stated \\
\hline Huang 2003 & $\begin{array}{l}\text { Therapy was started }<1 \text { day (ischaemic) or }<3 \text { days (haemorrhagic) } \\
\text { Both types of participants were analysed together as a group } \\
\text { Contact attempted for further details - this was unsuccessful }\end{array}$ \\
\hline Ishida 2001 & Uncertain methods - major imbalance in groups \\
\hline
\end{tabular}

Very early versus delayed mobilisation after stroke (Review) 


\begin{tabular}{|c|c|}
\hline Study & Reason for exclusion \\
\hline Kreisel 2005 & No time difference of therapy between groups \\
\hline Li 1999 & No details on timing \\
\hline Li 2003 & Timing not stated \\
\hline Li 2004 & Timing not stated \\
\hline Lin 2005 & Timing not stated, confounded by additional psychotherapy, cognitive therapy \\
\hline Liu 2001b & Rehabilitation started at 3 days \\
\hline Liu 2003b & Rehabilitation started at 3-5 days \\
\hline Liu 2004 & No control $<48$ hours, confounded by additional functional electrical stimulation \\
\hline Marshall 2011 & Insufficient details to confirm eligibility \\
\hline Miskovic 2004 & Insufficient details to confirm eligibility \\
\hline Pan 2004 & Timing not stated \\
\hline Qian 2003 & Timing not stated \\
\hline Qian 2004 & Timing not stated \\
\hline Raicevic 2000 & $\begin{array}{l}\text { Timing not stated } \\
\text { Possibly passive therapies - not mobilisation }\end{array}$ \\
\hline Richards 1993 & Gait rehabilitation began in first 7 days \\
\hline Sankara Kumaran 2013 & Not formally randomised - purposive sampling \\
\hline Song 2005 & Timing not stated \\
\hline Sun 2002 & Insufficient details to confirm eligibility \\
\hline Toyota 2001 & Not clearly randomised (major imbalance in groups) \\
\hline Truscott 1974 & Not an RCT (observational) \\
\hline Wang 2004 & Timing uncertain - recruited after stabilisation \\
\hline Wang 2005 & Treatment within 1 week of onset \\
\hline Wang 2006 & Rehabilitation began at $10-20$ days \\
\hline Wu 2012 & Delayed mobilisation - began on day 3 \\
\hline Xi 2003 & Quasi-randomised, uncertain when out of bed \\
\hline Xiao 2000 & Delayed mobilisation - recruited at day 3 \\
\hline Xiao 2004 & Timing not stated \\
\hline
\end{tabular}




\begin{tabular}{|c|c|}
\hline Study & Reason for exclusion \\
\hline Xie 2003a & Began at 6-52 hours \\
\hline Xue 2004 & $\begin{array}{l}\text { Intervention was the earlier delivery of a rehabilitation package that included mobilisation and } \\
\text { other therapies considered to potentially confound the results (speech therapy, swallowing thera- } \\
\text { py, psychological therapy including anti-depressants) }\end{array}$ \\
\hline Xue 2006 & Rehabilitation training began at 24 hours to 3 days \\
\hline Xue 2008 & Abstract only - no details on timing \\
\hline Zeng 2004 & Confounded by Chinese medicine, timing uncertain, quasi-randomised \\
\hline Zhang 1998 & Timing not stated \\
\hline Zhang 2001 & Began after 48 hours. Content uncertain \\
\hline Zhao 2003 & Uncertain timing: "as soon as state of illness was stable" \\
\hline Zheng 2004a & Began 3-7 days after stroke \\
\hline
\end{tabular}

$\mathrm{RCT}$ : randomised controlled trial

Characteristics of studies awaiting assessment [ordered by study ID]

Izumi 2001

\begin{tabular}{ll}
\hline Methods & Quote "RCT" \\
\hline Participants & Early rehabilitation \\
\hline Interventions & Uncertain timing of onset \\
\hline Outcomes & \\
\hline Notes & No further details \\
\hline
\end{tabular}

Liu 2010e

Methods Prospective multicenter, randomized controlled study, comparing standard care (SC) vs standard care plus early rehabilitation (VER: rehabilitation as soon as practical after randomization but within 48 hours of symptom onset)
Participants Patients presenting $<48$ hours after ICH (a first time ICH confirmed by MRI or CT) to the neurolo- gy wards or rehabilitation units of participating hospitals with no contraindications to being mo- bilised within 48 hours of stroke onset

Interventions

VER: participants commenced rehabilitation within 48 hours of ICH onset

SC group commenced rehabilitation after 7 days. Within the first week this involved bed rest or sitting in a chair. There is no showering or active rehabilitation with the main focus being medical management.

Standard rehabilitation is usually commenced 1 week after stroke admission and continues until discharge. It is performed by the participant's relatives under the guidance of medical staff and 
Liu 2010e (Continued)

usually involves 1) exercises of daily living, stretching exercises, and neuromuscular electric stimulation, and 2) functional training in which the participants are instructed to do repetitive and systematic practice of tasks, such as stirring, grasping, and pointing

\begin{tabular}{ll}
\hline Outcomes & Death, Barthel Index, SF-36, complications \\
\hline Notes & $\begin{array}{l}\text { Although rehabilitation started within } 48 \text { hours it appears to have been initially 'passive'. As yet we } \\
\text { have been unable to obtain further details from the authors. }\end{array}$ \\
\hline
\end{tabular}

Nilsson 2003

Methods Uncertain

\section{Participants}

Interventions

\section{Outcomes}

Notes Abstract only - no details

\section{Skevin 2009}

\begin{tabular}{ll}
\hline Methods & Uncertain \\
\hline Participants & Stroke patients within 24 hours \\
\hline Interventions & Early rehabilitation programme \\
\hline Outcomes & \\
\hline Notes & Abstract only - no clinical outcomes \\
\hline
\end{tabular}

\section{Song 2010}

\begin{tabular}{ll}
\hline Methods & Single-blinded, RCT \\
\hline Participants & Early stroke \\
\hline Interventions & Early rehabilitation \\
\hline Outcomes & \\
\hline Notes & Uncertain timing - trying to obtain full report \\
\hline
\end{tabular}

Xu 2001

Methods Uncertain


Xu 2001 (Continued)

Participants

\begin{tabular}{ll}
\hline Interventions & Early progressive physiotherapy \\
\hline Outcomes & \\
\hline Notes & Abstract only \\
\hline
\end{tabular}

\section{Zheng 2004}

Methods Uncertain

\section{Participants}

Interventions

\section{Outcomes}

Notes Unable to obtain abstract

\section{Zielke 2003}

\begin{tabular}{ll}
\hline Methods Uncertain \\
\hline Participants
\end{tabular}

\section{Participants}

Interventions

\section{Outcomes}

Notes No details available

CT: computed tomography

$\mathrm{ICH}$ : intracerebral haemorrhage

MRI: magnetic resonance imaging

$\mathrm{RCT}$ : randomized controlled trial

Characteristics of ongoing studies [ordered by study ID]

\section{AVERT-DOSE 2017}

\begin{tabular}{ll}
\hline Trial name or title & $\begin{array}{l}\text { AVERT-DOSE (Determining Optimal early rehabilitation after StrokE): a multi-arm covariate-adjust- } \\
\text { ed, response-adaptive randomized controlled trial }\end{array}$ \\
\hline Methods & Multicentre RCT \\
\hline Participants & Acute stroke patients (within 48 hours after stroke) \\
\hline Interventions & $\begin{array}{l}\text { Early mobility rehabilitation protocols informed by the world's first global trial of early rehabilita- } \\
\text { tion (AVERT) }\end{array}$ \\
\hline
\end{tabular}


AVERT-DOSE 2017 (Continued)

\begin{tabular}{ll} 
Outcomes & Rankin score \\
\hline Starting date & Proposal at planning stage (2018 to 2022) \\
\hline Contact information & Julie Bernhardt, Florey Institute of Neuroscience and Mental Health, Melbourne, Australia \\
\hline Notes &
\end{tabular}

\section{ChiCTR-ICR-15005992}

\begin{tabular}{ll}
\hline Trial name or title & Effect of early and intensive rehabilitation on functional recovery after stroke \\
\hline Methods & $\begin{array}{l}\text { Randomised parallel controlled comparison of } 2 \text { levels of early rehabilitation compared with con- } \\
\text { ventional rehabilitation }\end{array}$ \\
\hline Participants & Ischaemic stroke available for randomization within 24 hours of symptom onset \\
\hline Interventions & 2 levels of early rehabilitation compared with conventional rehabilitation \\
\hline Outcomes & $\begin{array}{l}\text { MRI scan, NIHSS score, Fugl-Meyer Assessment Scale, Rivermead Motor Assessment Scale, Barthel } \\
\text { Index }\end{array}$ \\
\hline Starting date & $\begin{array}{l}\text { Yanna Tong, Department of Neurology, Beijing Luhe Hospital, Capital Medical University, Tongzhou } \\
\text { District, Beijing, China } \\
\text { tongyanna@163.com }+86 \text { 13426364922 }\end{array}$ \\
\hline Notes &
\end{tabular}

\section{ChiCTR-IPR-16008652}

Trial name or title Efficacy and safety of very early rehabilitation within 24 hours of ischemic stroke with small-artery occlusion

\begin{tabular}{ll}
\hline Methods & RCT \\
\hline Participants & $\begin{array}{l}\text { Inclusion criteria: patients } 18 \text { years or older consecutively admitted to the stroke unit within } 24 \\
\text { hours after stroke onset were screened for recruitment. Patients with cerebral infarction and small } \\
\text { artery occlusion, defined according to the World Health Organization definition, were all included } \\
\text { Exclusion criteria: patients with mRS score } \geq 1 \text { on admission; patients who were unable to com- } \\
\text { plete the baseline survey because of serious aphasia, language difficulties, or cognitive deficits; pa- } \\
\text { tient with other medical conditions, such as severe heart failure, acute coronary syndrome, which } \\
\text { prevented early rehabilitation; and patients who were unable to provide informed consent }\end{array}$ \\
\hline Interventions & Very early rehabilitation (within 24 hours) versus early rehabilitation \\
\hline Outcomes & NIHSS; Fugl-Meyer Assessment; Barthel Index; mRS \\
\hline Starting date & 2016 \\
\hline Contact information & shoufengliu2010@163.com
\end{tabular}


ChicTR-IPR-16008652 (Continued)

6 Jizhao Road, Jinnan District, Tianjin, China

Telephone: +86 022-59065182

Email: shoufengliu2010@163.com

Affiliation: Tianjin Huanhu Hospital

Notes Aim to recruit 2 groups of 50 participants each

\section{ChiCTR-TRC-08000201}

\begin{tabular}{ll}
\hline Trial name or title & The effect of early mobilisation for stroke patients \\
\hline Methods & Randomised trial \\
\hline Participants & The effect of early mobilisation for stroke patients \\
\hline Interventions & VEM (within 48 hrs) vs early mobilisation (1 week) after stroke \\
\hline Outcomes & \\
\hline Starting date & \\
\hline Contact information & Onging with at least 150 participants planned \\
\hline Notes &
\end{tabular}

mRS: modified Rankin scale

NIHSS: National Institutes of Health Stroke Scale

RCT: randomized controlled trial

VEM: very early mobilisation

\section{DATA AND ANALYSES}

Comparison 1. Very early mobilisation (VEM) versus standard care (measured at end of scheduled follow-up)

\begin{tabular}{lllll}
\hline Outcome or subgroup title & No. of studies & $\begin{array}{l}\text { No. of partici- } \\
\text { pants }\end{array}$ & Statistical method & Effect size \\
\hline 1 Death or poor outcome & 8 & 2542 & Odds Ratio (M-H, Fixed, 95\% Cl) & $1.08[0.92,1.26]$ \\
\hline 2 Death & 8 & 2561 & Odds Ratio (M-H, Fixed, 95\% Cl) & $1.27[0.95,1.70]$ \\
\hline $\begin{array}{l}\text { 3 Death or dependence (modified } \\
\text { Rankin score 3 to 6) }\end{array}$ & 8 & 2542 & Odds Ratio (M-H, Fixed, 95\% Cl) & $1.08[0.92,1.26]$ \\
\hline $\begin{array}{l}\text { 4 Death or institutional care } \\
\text { 5 Activities of daily living (ADL) }\end{array}$ & 9 & 227 & Odds Ratio (M-H, Fixed, 95\% Cl) & $1.05[0.53,2.07]$ \\
\hline score & 2630 & & Mean Difference (IV, Random, & $1.94[0.75,3.13]$ \\
\hline
\end{tabular}




\begin{tabular}{|c|c|c|c|c|}
\hline Outcome or subgroup title & No. of studies & $\begin{array}{l}\text { No. of partici- } \\
\text { pants }\end{array}$ & Statistical method & Effect size \\
\hline 6 Subjective Health Status score & 1 & 68 & $\begin{array}{l}\text { Mean Difference (IV, Random, } \\
95 \% \mathrm{CI})\end{array}$ & $0.07[-0.10,0.23]$ \\
\hline 7 Able to walk & 4 & 2255 & Odds Ratio (M-H, Fixed, 95\% Cl) & $1.00[0.83,1.21]$ \\
\hline 8 Mobility score & 2 & 102 & $\begin{array}{l}\text { Std. Mean Difference (IV, Ran- } \\
\text { dom, } 95 \% \mathrm{CI})\end{array}$ & $0.14[-0.27,0.56]$ \\
\hline $\begin{array}{l}9 \text { Any complication: participants } \\
\text { who experienced at least one } \\
\text { complication }\end{array}$ & 7 & 2778 & Odds Ratio (M-H, Fixed, 95\% CI) & $0.88[0.73,1.06]$ \\
\hline $\begin{array}{l}10 \text { Type of complication: partic- } \\
\text { ipants who experienced at least } \\
\text { one complication }\end{array}$ & 7 & & Odds Ratio (M-H, Fixed, 95\% CI) & Subtotals only \\
\hline 10.1 Complications of immobility & 7 & 2778 & Odds Ratio (M-H, Fixed, 95\% Cl) & $0.79[0.60,1.03]$ \\
\hline 10.2 Other complications & 6 & 2435 & Odds Ratio (M-H, Fixed, 95\% CI) & $0.98[0.78,1.23]$ \\
\hline 11 Mood score & 2 & 100 & $\begin{array}{l}\text { Std. Mean Difference (IV, Ran- } \\
\text { dom, } 95 \% \mathrm{Cl} \text { ) }\end{array}$ & $0.07[-0.33,0.46]$ \\
\hline $\begin{array}{l}12 \text { Length of acute hospital stay } \\
\text { (days) }\end{array}$ & 8 & 2551 & $\begin{array}{l}\text { Mean Difference (IV, Random, } \\
95 \% \mathrm{CI})\end{array}$ & $-1.44[-2.28,-0.60]$ \\
\hline
\end{tabular}

Analysis 1.1. Comparison 1 Very early mobilisation (VEM) versus standard care (measured at end of scheduled follow-up), Outcome 1 Death or poor outcome.

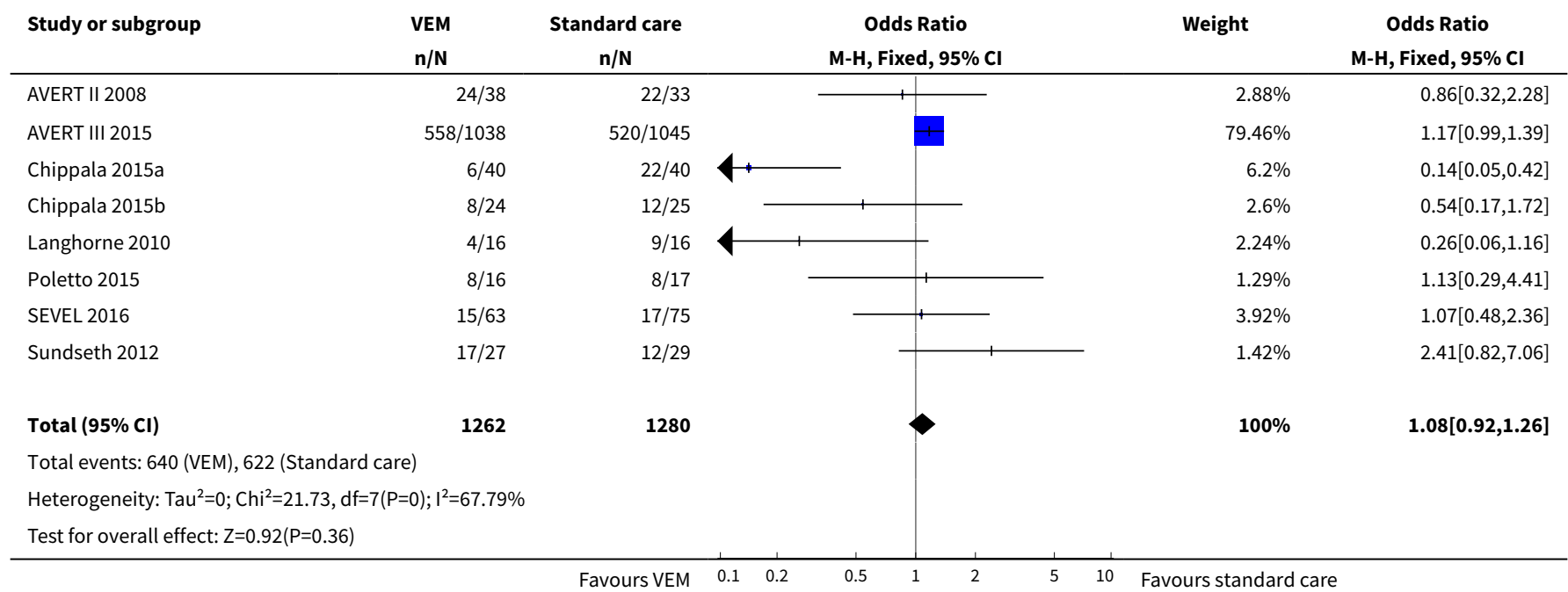


Analysis 1.2. Comparison 1 Very early mobilisation (VEM) versus standard care (measured at end of scheduled follow-up), Outcome 2 Death.

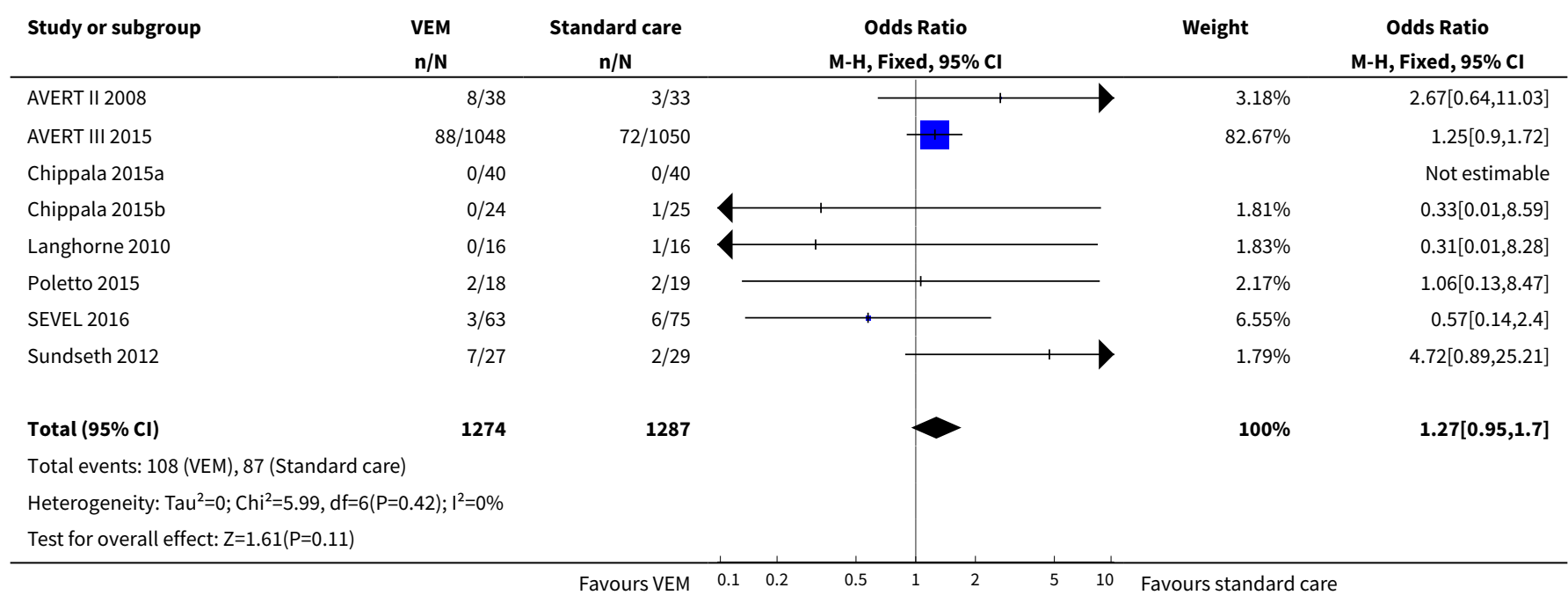

Analysis 1.3. Comparison 1 Very early mobilisation (VEM) versus standard care (measured at end of scheduled follow-up), Outcome 3 Death or dependence (modified Rankin score 3 to 6).

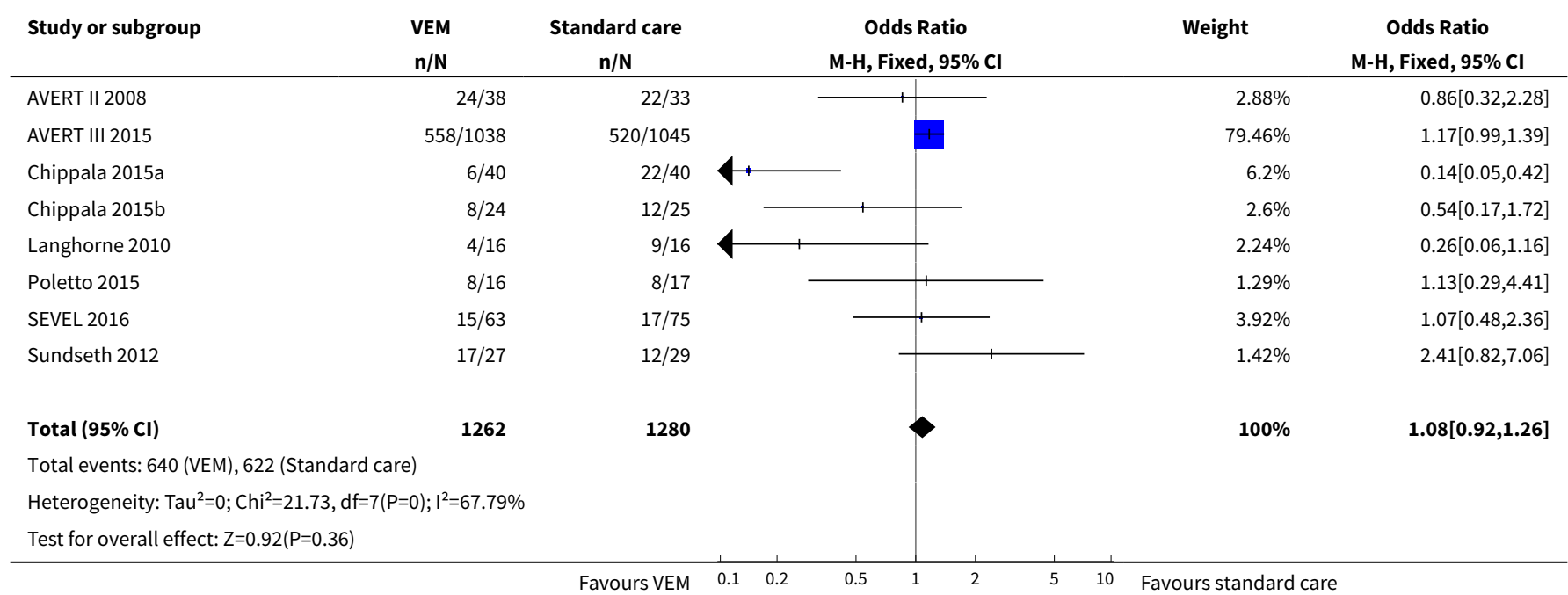

Analysis 1.4. Comparison 1 Very early mobilisation (VEM) versus standard care (measured at end of scheduled follow-up), Outcome 4 Death or institutional care.

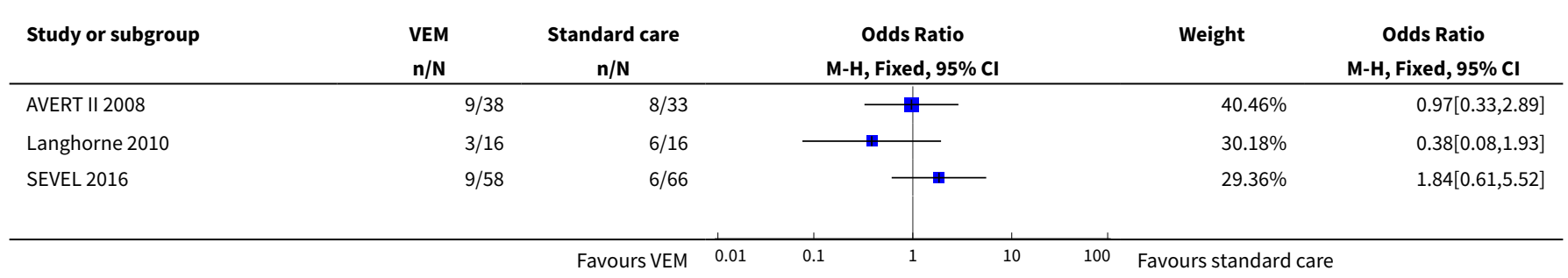




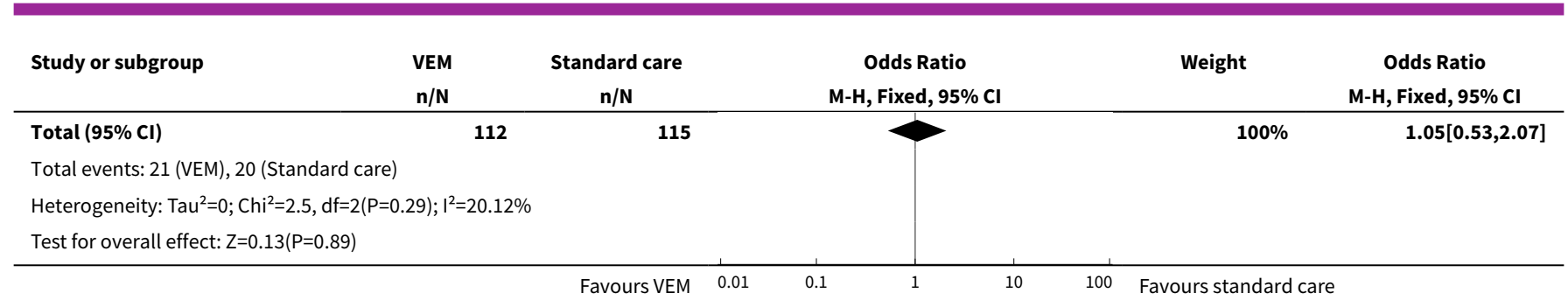

Analysis 1.5. Comparison 1 Very early mobilisation (VEM) versus standard care (measured at end of scheduled follow-up), Outcome 5 Activities of daily living (ADL) score.

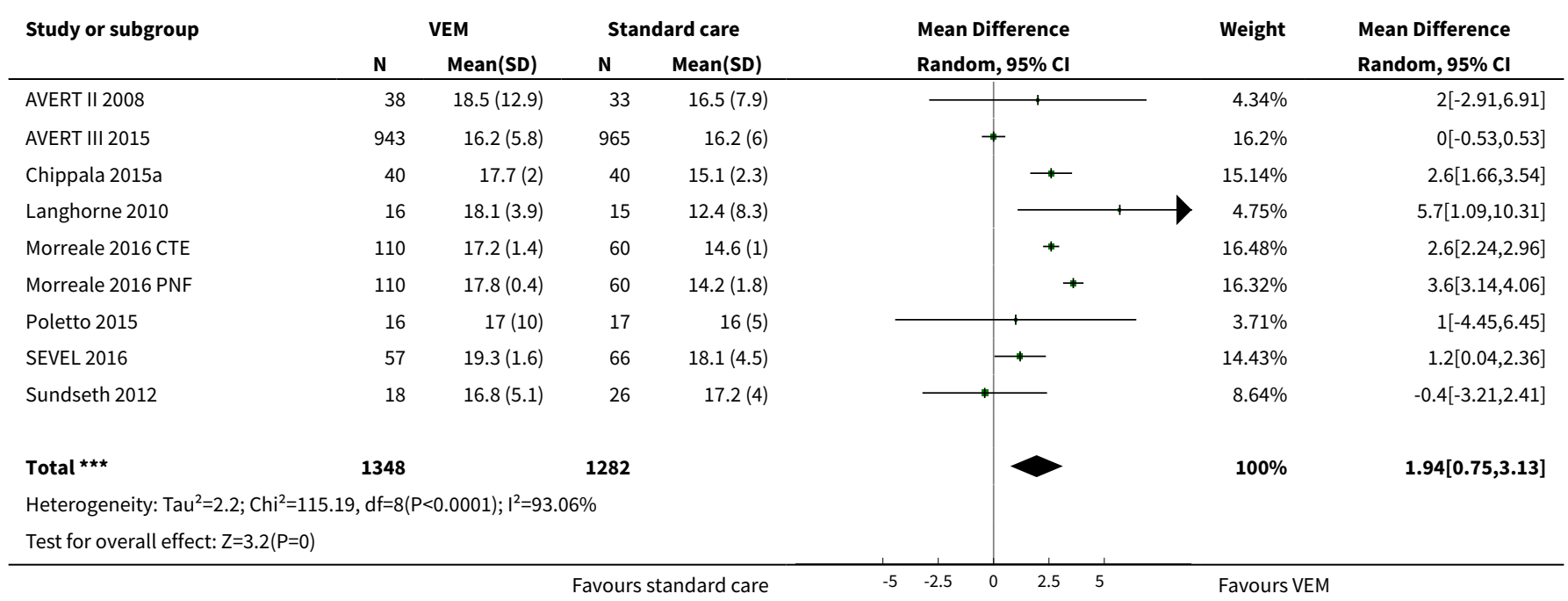

Analysis 1.6. Comparison 1 Very early mobilisation (VEM) versus standard care (measured at end of scheduled follow-up), Outcome 6 Subjective Health Status score.

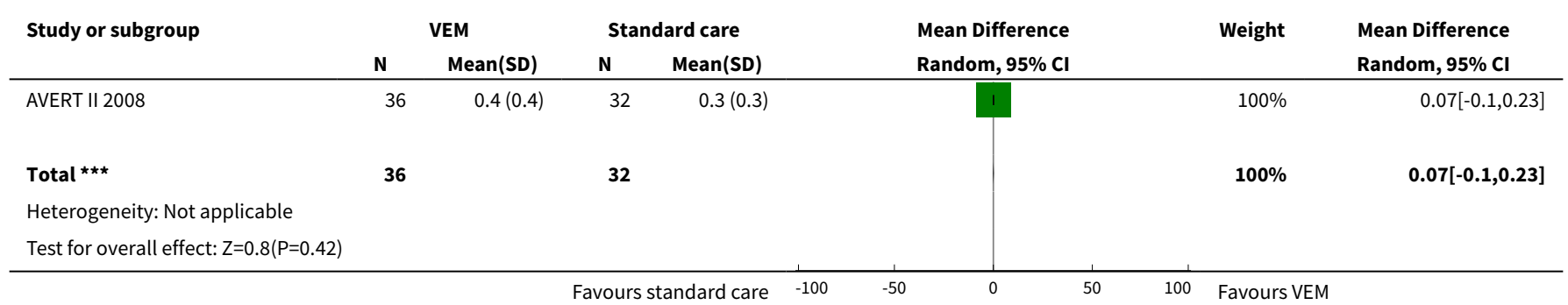

Analysis 1.7. Comparison 1 Very early mobilisation (VEM) versus standard care (measured at end of scheduled follow-up), Outcome 7 Able to walk.

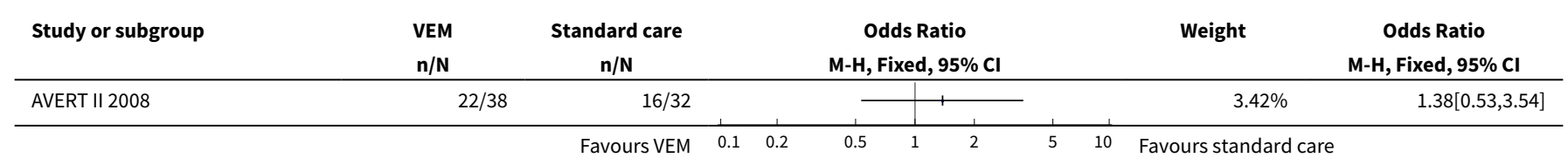




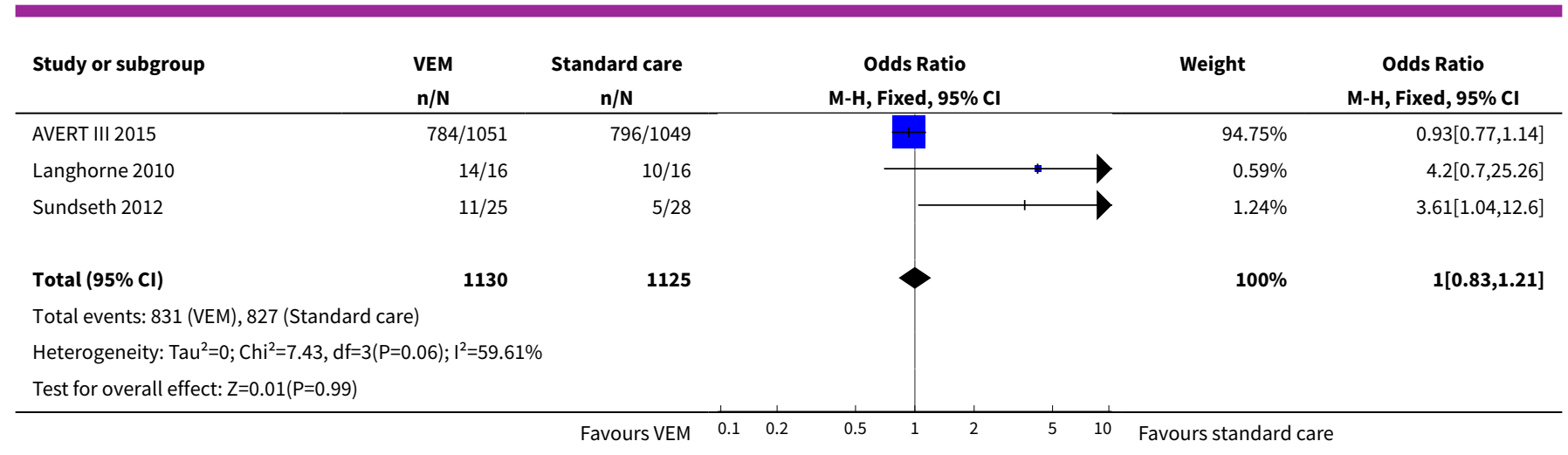

Analysis 1.8. Comparison 1 Very early mobilisation (VEM) versus standard care (measured at end of scheduled follow-up), Outcome 8 Mobility score.

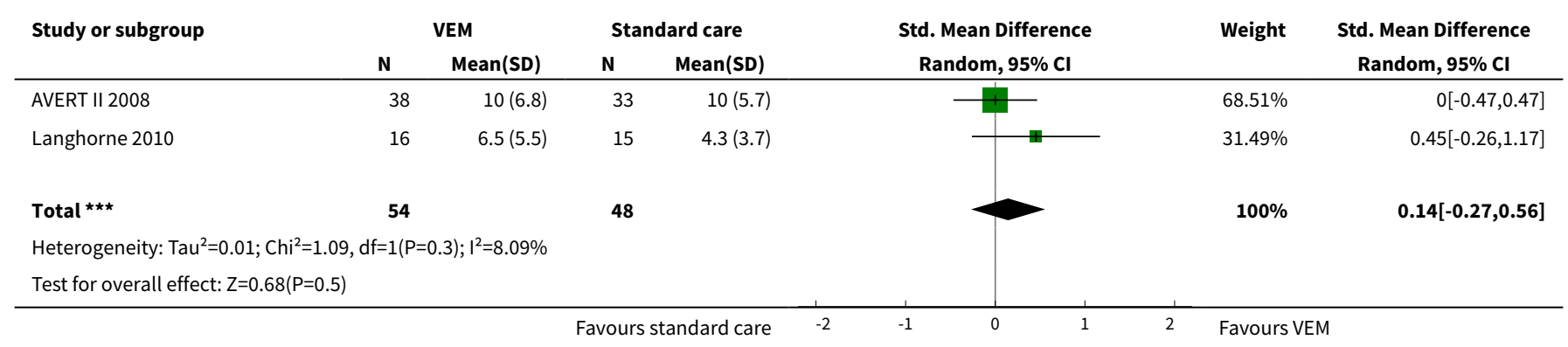

Analysis 1.9. Comparison 1 Very early mobilisation (VEM) versus standard care (measured at end of scheduled follow-up), Outcome 9 Any complication: participants who experienced at least one complication.

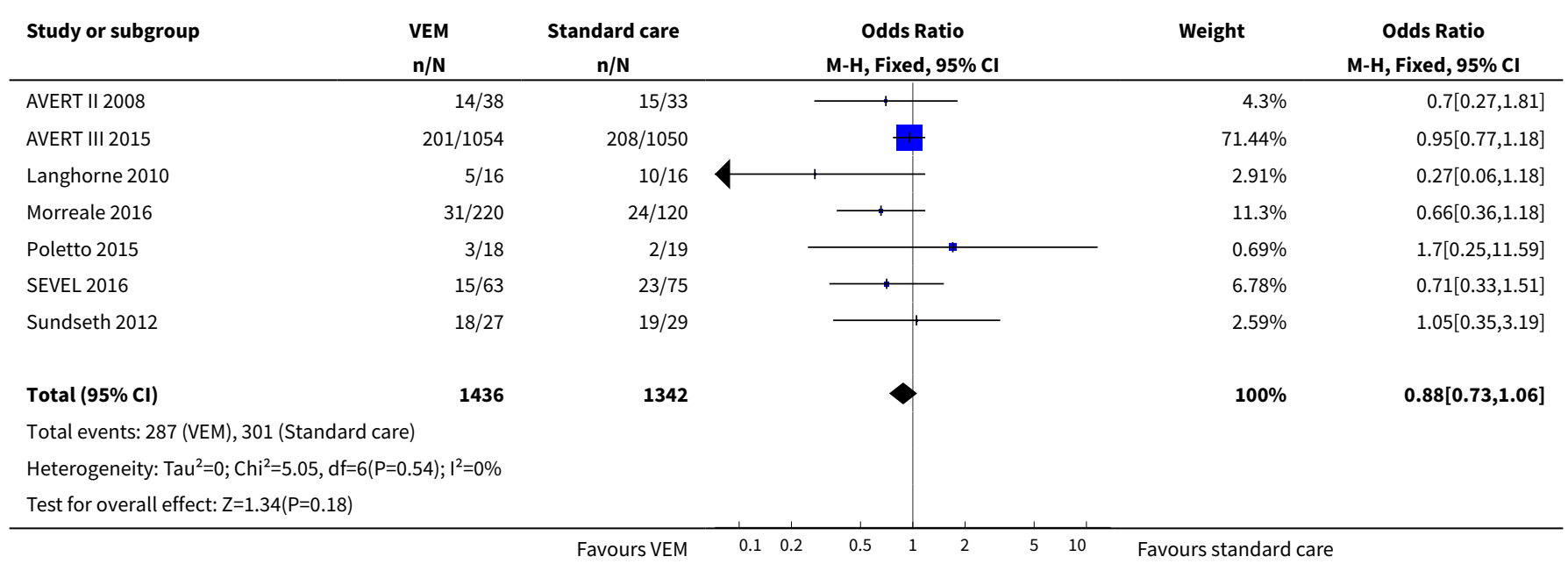


Analysis 1.10. Comparison 1 Very early mobilisation (VEM) versus standard care (measured at end of scheduled follow-up), Outcome 10 Type of complication: participants who experienced at least one complication.

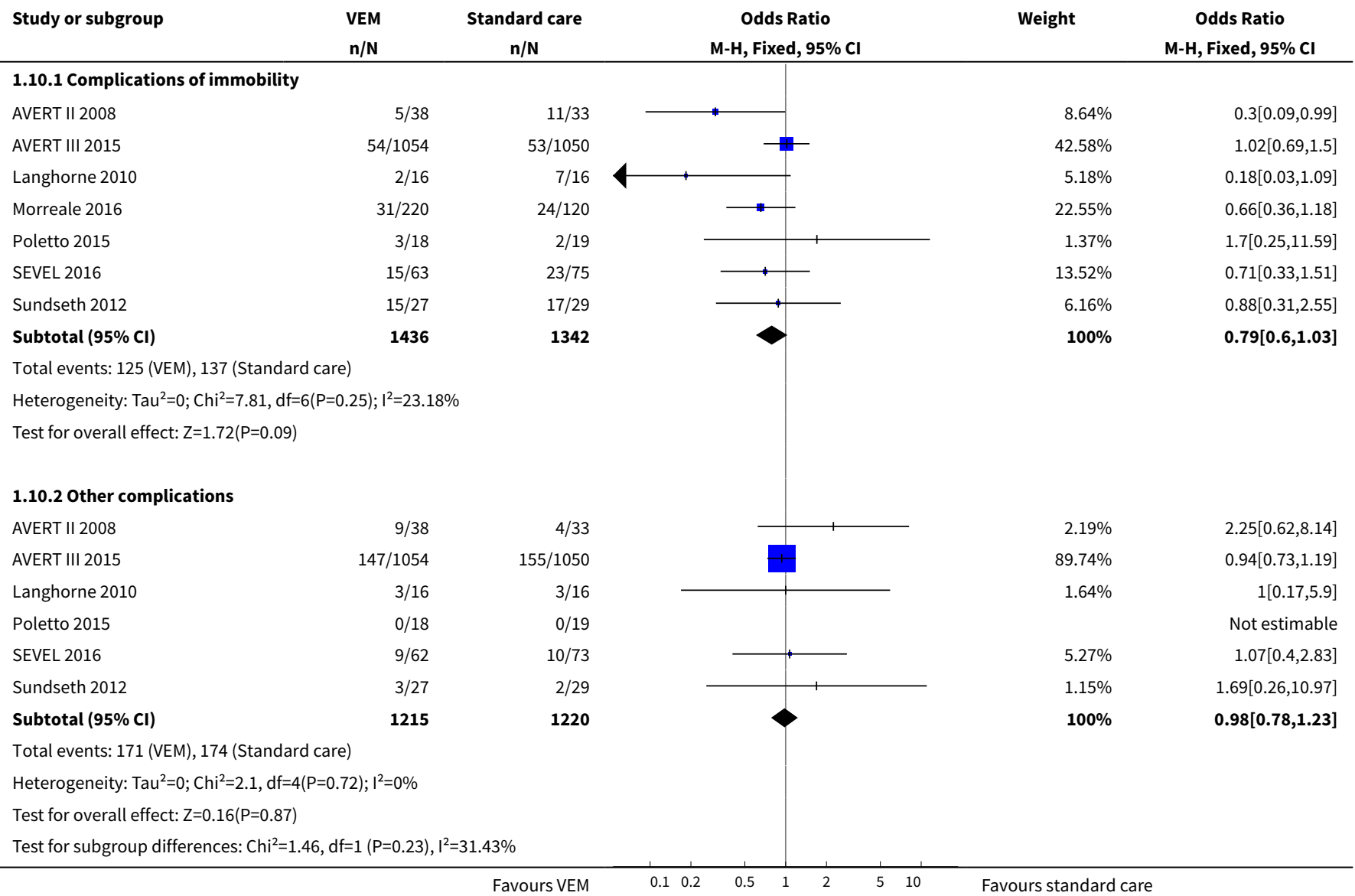

Analysis 1.11. Comparison 1 Very early mobilisation (VEM) versus standard care (measured at end of scheduled follow-up), Outcome 11 Mood score.

\begin{tabular}{|c|c|c|c|c|c|c|c|}
\hline \multirow[t]{2}{*}{ Study or subgroup } & \multicolumn{2}{|c|}{ VEM } & \multicolumn{2}{|c|}{ Standard care } & \multirow{2}{*}{$\begin{array}{c}\text { Std. Mean Difference } \\
\text { Random, } 95 \% \mathrm{Cl}\end{array}$} & \multirow[t]{2}{*}{ Weight } & \multirow{2}{*}{$\begin{array}{c}\text { Std. Mean Difference } \\
\text { Random, } 95 \% \mathrm{Cl}\end{array}$} \\
\hline & $\mathbf{N}$ & Mean(SD) & $\mathbf{N}$ & Mean(SD) & & & \\
\hline AVERT II 2008 & 21 & $12.4(9.1)$ & 23 & $12.6(6.6)$ & +1 & $44.04 \%$ & $-0.02[-0.62,0.57]$ \\
\hline Sundseth 2012 & 27 & $11(9.8)$ & 29 & $9.9(5.3)$ & & $55.96 \%$ & $0.14[-0.39,0.66]$ \\
\hline Total ${ }^{\star \star \star}$ & 48 & & 52 & & & $100 \%$ & $0.07[-0.33,0.46]$ \\
\hline \multicolumn{8}{|c|}{ Heterogeneity: $\mathrm{Tau}^{2}=0 ; \mathrm{Chi}^{2}=0.16, \mathrm{df}=1(\mathrm{P}=0.69) ; \mathrm{I}^{2}=0 \%$} \\
\hline
\end{tabular}


Analysis 1.12. Comparison 1 Very early mobilisation (VEM) versus standard care (measured at end of scheduled follow-up), Outcome 12 Length of acute hospital stay (days).

\begin{tabular}{|c|c|c|c|c|c|c|c|}
\hline \multirow{3}{*}{$\begin{array}{l}\text { Study or subgroup } \\
\text { AVERT II } 2008\end{array}$} & \multicolumn{2}{|c|}{ VEM } & \multicolumn{2}{|c|}{ Standard care } & \multirow{2}{*}{$\begin{array}{l}\text { Mean Difference } \\
\text { Random, 95\% Cl }\end{array}$} & \multirow[t]{2}{*}{ Weight } & \multirow{2}{*}{$\begin{array}{l}\text { Mean Difference } \\
\text { Random, } 95 \% \mathrm{Cl}\end{array}$} \\
\hline & $\mathbf{N}$ & $\operatorname{Mean}(S D)$ & $\mathbf{N}$ & Mean(SD) & & & \\
\hline & 38 & $8.6(9.4)$ & 33 & $9.8(8)$ & 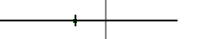 & $4.01 \%$ & $-1.2[-5.25,2.85]$ \\
\hline AVERT III 2015 & 1052 & $11.6(16.3)$ & 1050 & $12(16.1)$ & $\longrightarrow$ & $21.75 \%$ & $-0.4[-1.79,0.99]$ \\
\hline Chippala 2015a & 40 & $8(1.4)$ & 40 & $10.3(3.4)$ & —- & $26.84 \%$ & $-2.25[-3.39,-1.11]$ \\
\hline Chippala 2015b & 24 & $8.7(1.4)$ & 25 & $11.2(3.2)$ & 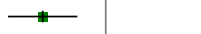 & $21.96 \%$ & $-2.5[-3.87,-1.13]$ \\
\hline Langhorne 2010 & 16 & $11.3(9.9)$ & 16 & $14.9(15.3)$ & & $0.88 \%$ & $-3.6[-12.53,5.33]$ \\
\hline SEVEL 2016 & 58 & $9.8(4.9)$ & 66 & $10.5(6.1)$ & \begin{tabular}{l|l}
$\longrightarrow$ & \\
\end{tabular} & $13.92 \%$ & $-0.7[-2.64,1.24]$ \\
\hline Sundseth 2012 & 27 & $10.2(4.2)$ & 29 & $9.9(5.3)$ & & $9.38 \%$ & $0.3[-2.2,2.8]$ \\
\hline Total $* \star \star$ & 1273 & & 1278 & & & $100 \%$ & $-1.44[-2.28,-0.6]$ \\
\hline \multicolumn{8}{|c|}{ Heterogeneity: $\mathrm{Tau}^{2}=0.35 ; \mathrm{Chi}^{2}=9.44, \mathrm{df}=7(\mathrm{P}=0.22) ; \mathrm{I}^{2}=25.84 \%$} \\
\hline \multicolumn{3}{|c|}{ Test for overall effect: $Z=3.35(P=0)$} & & & & & \\
\hline
\end{tabular}

\section{Comparison 2. Very early mobilisation versus standard care (results at 3 months)}

\begin{tabular}{lllll}
\hline Outcome or subgroup title & No. of studies & $\begin{array}{l}\text { No. of partici- } \\
\text { pants }\end{array}$ & Statistical method & Effect size \\
\hline 1 Death or poor outcome & 8 & 2542 & Odds Ratio (M-H, Fixed, 95\% Cl) & $1.08[0.92,1.26]$ \\
\hline 2 Death & 8 & 2570 & Odds Ratio (M-H, Fixed, 95\% Cl) & $1.27[0.95,1.70]$ \\
\hline $\begin{array}{l}\text { 3 Death or dependence (modi- } \\
\text { fied Rankin score 3 to 6) }\end{array}$ & 8 & 2542 & Odds Ratio (M-H, Fixed, 95\% Cl) & $1.08[0.92,1.26]$ \\
\hline $\begin{array}{l}\text { 4 Death or institutional care } \\
\text { 5 Activities of daily living (ADL) }\end{array}$ & 9 & 227 & Odds Ratio (M-H, Fixed, 95\% Cl) & $1.05[0.53,2.07]$ \\
\hline \begin{tabular}{l} 
score \\
\hline
\end{tabular} & 2634 & & $\begin{array}{l}\text { Mean Difference (IV, Random, } \\
\text { 95\% Cl) }\end{array}$ & $0.75[0.01,1.49]$ \\
\end{tabular}

Analysis 2.1. Comparison 2 Very early mobilisation versus standard care (results at 3 months), Outcome 1 Death or poor outcome.

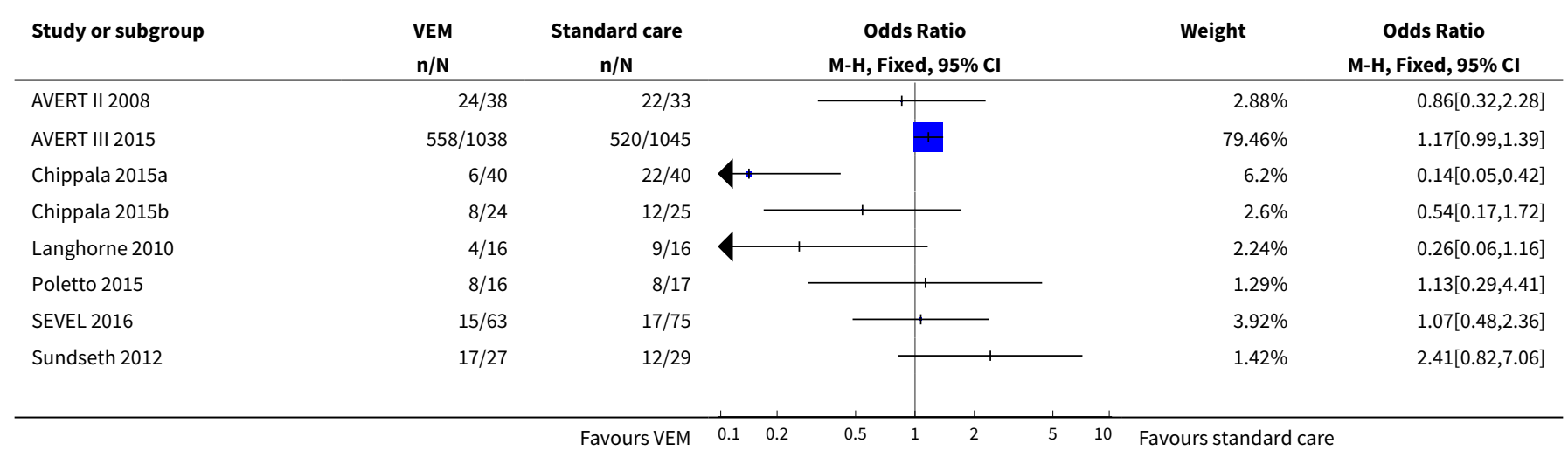




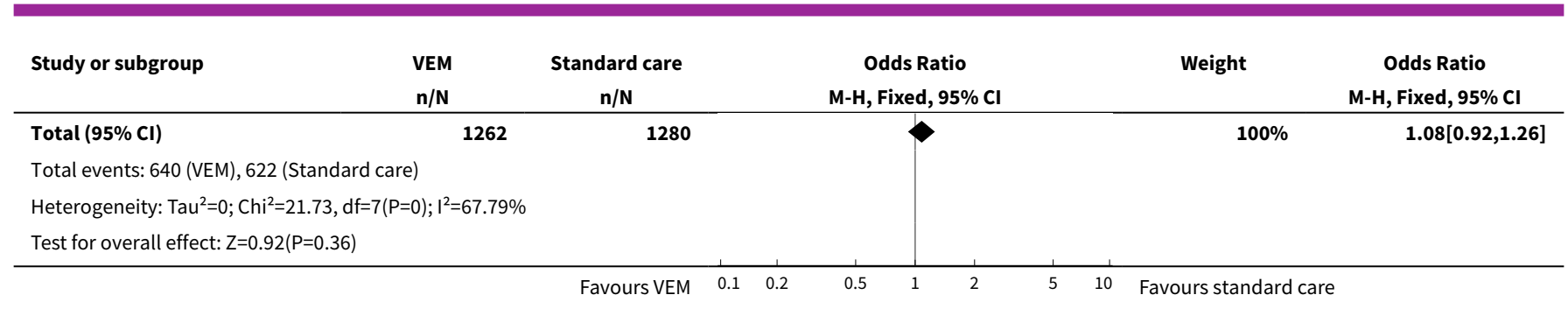

Analysis 2.2. Comparison 2 Very early mobilisation versus standard care (results at 3 months), Outcome 2 Death.

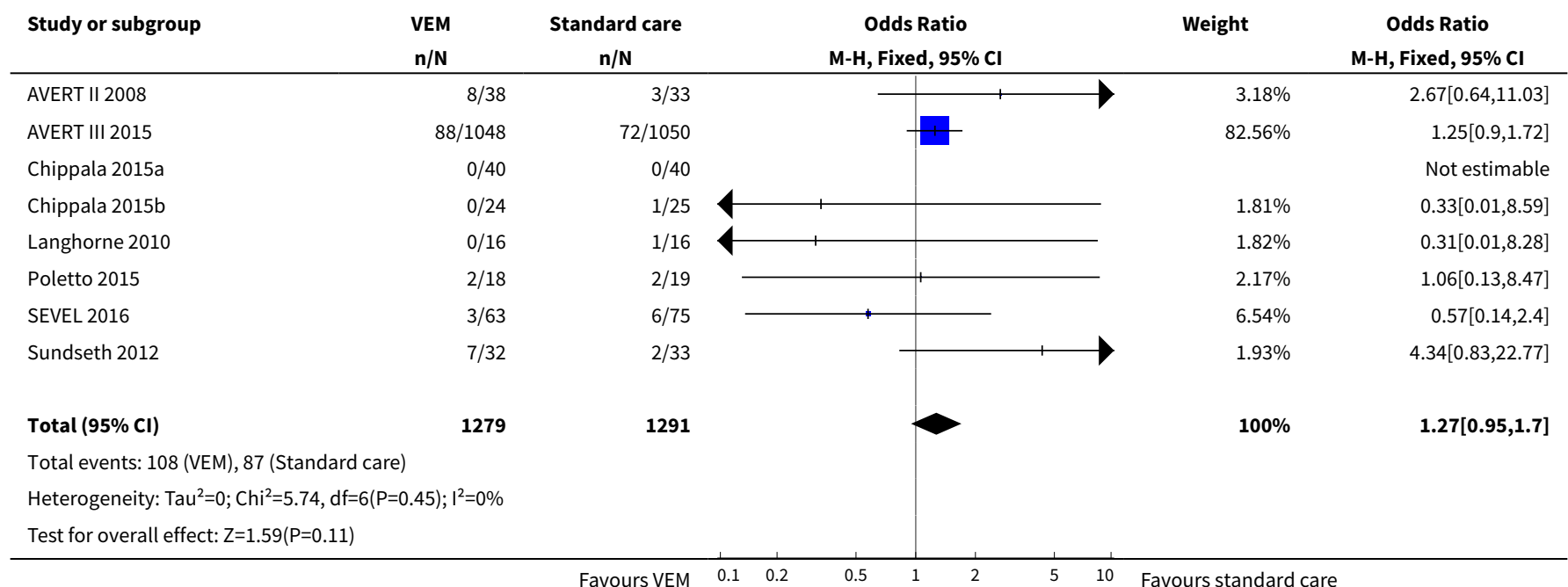

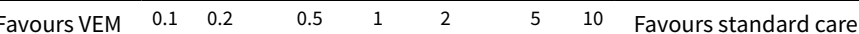

Analysis 2.3. Comparison 2 Very early mobilisation versus standard care (results at 3 months), Outcome 3 Death or dependence (modified Rankin score 3 to 6 ).

\begin{tabular}{|c|c|c|c|c|c|}
\hline Study or subgroup & $\begin{array}{l}\text { VEM } \\
\text { n/N }\end{array}$ & $\begin{array}{c}\text { Standard care } \\
n / N\end{array}$ & $\begin{array}{c}\text { Odds Ratio } \\
\text { M-H, Fixed, } 95 \% \mathrm{Cl}\end{array}$ & Weight & $\begin{array}{c}\text { Odds Ratio } \\
\text { M-H, Fixed, } 95 \% \mathrm{Cl}\end{array}$ \\
\hline AVERT II 2008 & $24 / 38$ & $22 / 33$ & & $2.88 \%$ & $0.86[0.32,2.28]$ \\
\hline AVERT III 2015 & $558 / 1038$ & $520 / 1045$ & & $79.46 \%$ & $1.17[0.99,1.39]$ \\
\hline Chippala 2015a & $6 / 40$ & $22 / 40$ & & $6.2 \%$ & $0.14[0.05,0.42]$ \\
\hline Chippala 2015b & $8 / 24$ & $12 / 25$ & & $2.6 \%$ & $0.54[0.17,1.72]$ \\
\hline Langhorne 2010 & $4 / 16$ & $9 / 16$ & & $2.24 \%$ & $0.26[0.06,1.16]$ \\
\hline SEVEL 2016 & $15 / 63$ & $17 / 75$ & - & $3.92 \%$ & $1.07[0.48,2.36]$ \\
\hline Sundseth 2012 & $17 / 27$ & $12 / 29$ & & $1.42 \%$ & $2.41[0.82,7.06]$ \\
\hline Total $(95 \% \mathrm{Cl})$ & 1262 & 1280 & & $100 \%$ & $1.08[0.92,1.26]$ \\
\hline \multicolumn{6}{|c|}{ Total events: 640 (VEM), 622 (Standard care) } \\
\hline \multicolumn{6}{|c|}{ Heterogeneity: $\operatorname{Tau}^{2}=0 ; \mathrm{Chi}^{2}=21.73, \mathrm{df}=7(\mathrm{P}=0) ; \mathrm{I}^{2}=67.79 \%$} \\
\hline
\end{tabular}


Analysis 2.4. Comparison 2 Very early mobilisation versus standard care (results at 3 months), Outcome 4 Death or institutional care.

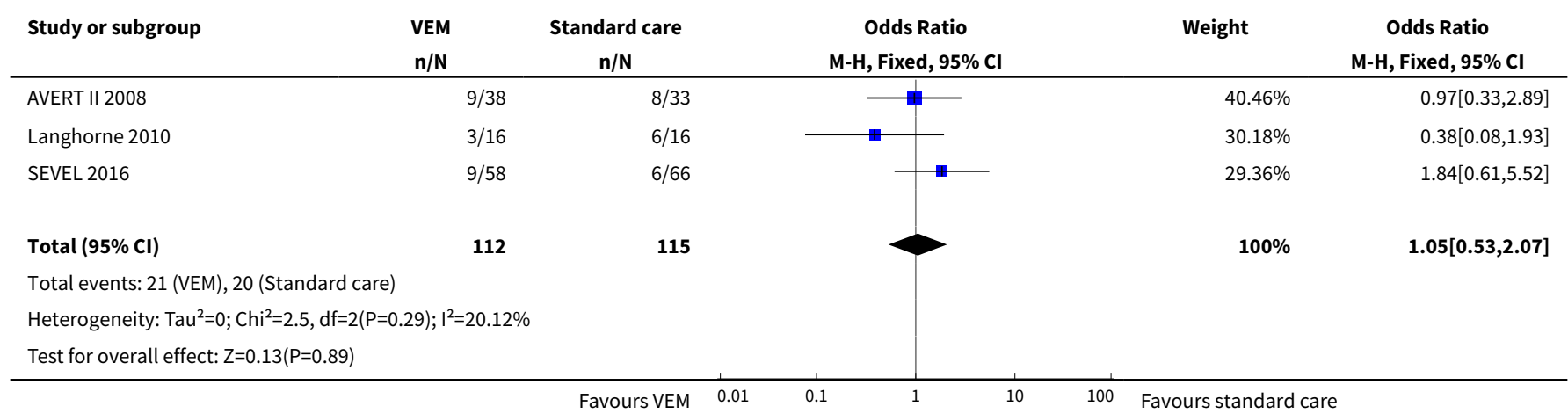

Analysis 2.5. Comparison 2 Very early mobilisation versus standard care (results at 3 months), Outcome 5 Activities of daily living (ADL) score.

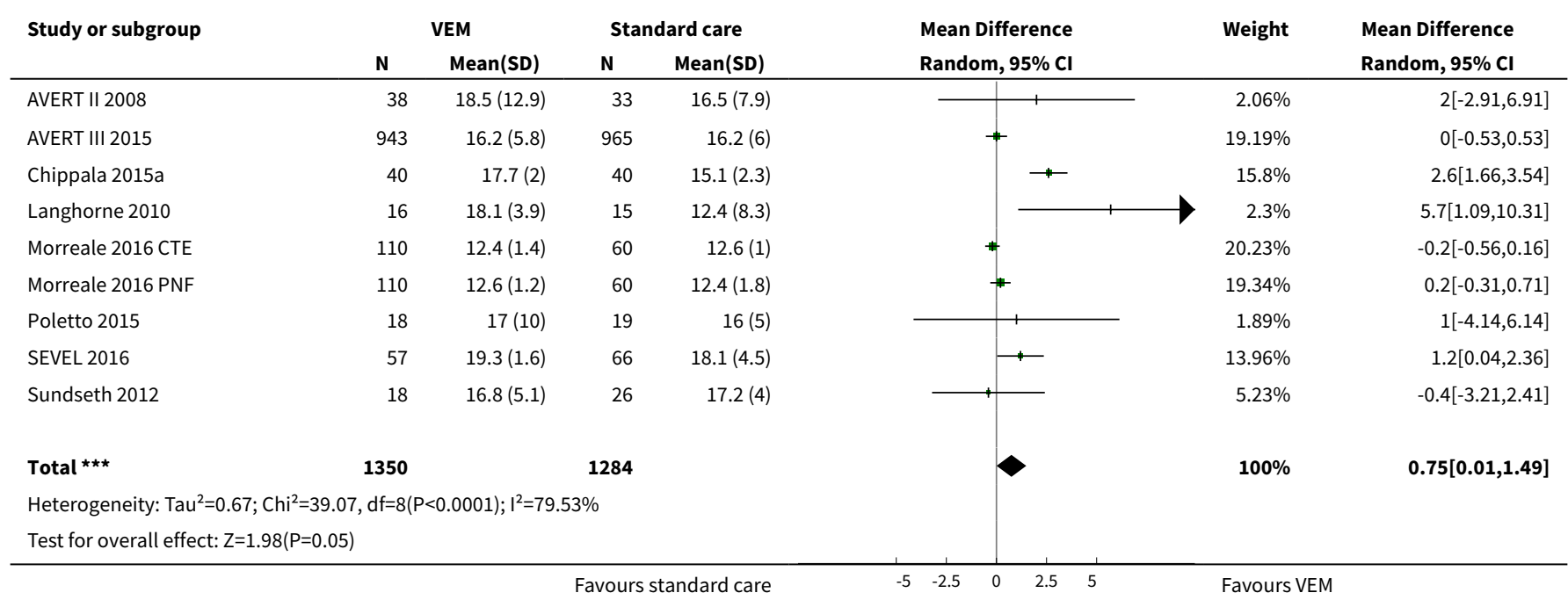




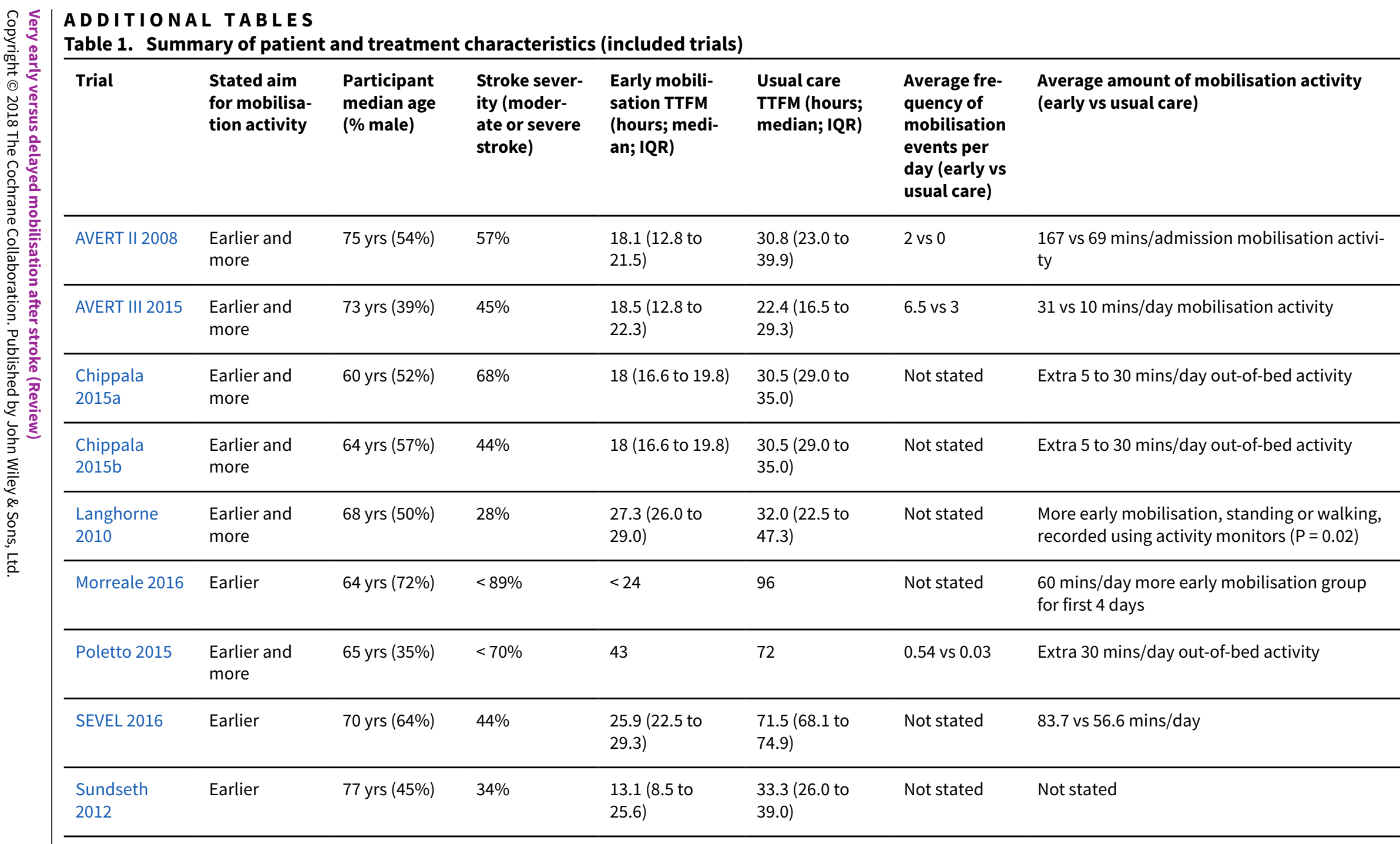

IQR: interquartile range

mins: minutes

TTFM: time from stroke to first mobilisation activity (hours)

vs: versus

yrs: years 
Table 2. Time-to-first mobilisation (TTFM) comparisons (included trials)

\begin{tabular}{|c|c|c|c|c|c|}
\hline \multirow{2}{*}{$\begin{array}{l}\text { Usual care group } \\
\text { TTFM characteristics }\end{array}$} & \multicolumn{5}{|c|}{ Very early mobilisation group TTFM characteristics } \\
\hline & 12 hours & 18 hours & 24 hours & $>\mathbf{3 0}$ hours & $>48$ hours \\
\hline 12 hours & - & - & - & - & - \\
\hline 18 hours & - & - & - & - & - \\
\hline 24 hours & - & AVERT III 2015 (2014 ppts) & - & - & - \\
\hline$>30$ hours & $\begin{array}{l}\text { Sundseth } \\
2012 \text { (65 ppts) }\end{array}$ & $\begin{array}{l}\text { AVEAVERT II 2008; Chippala } \\
\text { 2015a; Chippala 2015b ( } 211 \\
\text { ppts) }\end{array}$ & $\begin{array}{l}\text { Langhorne } 2010 \\
\text { (32 ppts) }\end{array}$ & - & - \\
\hline$>48$ hours & & & $\begin{array}{l}\text { SEVEL } 2016167 \\
\text { ppts) }\end{array}$ & $\begin{array}{l}\text { Poletto } 2015 \\
\text { (39 ppts) }\end{array}$ & - \\
\hline
\end{tabular}

ppts = participants

Table shows Time-to-first mobilisation (TTFM) in each trial with the very early mobilisation TTFM group in the columns and usual care TTFM in the rows. The number of trials (participants) in each direct comparison of TTFM are also shown. For example Sundseth 2012 compared TTFM of approximately 12 hours with $>30$ hours and included 65 participants.

We did not include data from Morreale 2016 in this analysis as we did not have access to dichotomous data on poor outcome or death

Table 3. Inconsistency table for poor outcome (death or dependency at 3 months)

\begin{tabular}{|c|c|c|c|c|c|c|}
\hline $\begin{array}{l}\text { TTFM cate- } \\
\text { gory }\end{array}$ & $\begin{array}{l}\text { TTFM record- } \\
\text { ed in the trials } \\
\text { (median; IQR) }\end{array}$ & $\begin{array}{l}\text { Direct } \\
\text { compari- } \\
\text { son } \\
\text { (OR) }\end{array}$ & $\begin{array}{l}\text { Indirect } \\
\text { compari- } \\
\text { son } \\
\text { (OR) }\end{array}$ & $\begin{array}{l}\text { Log difference }(95 \% \mathrm{CI}) \\
\text { between direct and indi- } \\
\text { rect comparisons }\end{array}$ & $\begin{array}{l}\text { P value of dif- } \\
\text { ference be- } \\
\text { tween direct } \\
\text { and indirect } \\
\text { comparisons }\end{array}$ & $\begin{array}{l}\text { Network meta- } \\
\text { analysis } \\
\text { (OR and } 95 \% \mathrm{Cl} \text { ) }\end{array}$ \\
\hline 12 hours & 13 (9 to 26$)$ & NA & 6.62 & NA & NA & 6.61 (1.36 to 32.09$)$ \\
\hline 18 hours & 18 (13 to 21$)$ & 1.17 & 0.80 & $-0.39(-2.09$ to 1.31$)$ & 0.65 & 1.07 (0.53 to 2.19$)$ \\
\hline 24 hours & 26 (22 to 29 ) & $\begin{array}{l}1.00 \text { (refer- } \\
\text { ence) }\end{array}$ & Reference & Reference & Reference & Reference \\
\hline$>30$ hours & 32 (26 to 40$)$ & 3.86 & 2.46 & $0.45(-1.50$ to 2.41$)$ & 0.65 & 2.74 (1.18 to 6.37 ) \\
\hline$>48$ hours & 72 (68 to 75$)$ & 0.94 & 3.03 & $-1.18(-3.33$ to 0.98$)$ & 0.28 & $1.29(0.50$ to 3.37$)$ \\
\hline
\end{tabular}

The first two columns show the TTFM category plus the actual recorded TTFM for that category.

The next two columns show the odds ratio of a poor outcome for the direct and indirect comparison of the TTFM category, with 24 hours as the reference category.

The fifth column shows the log difference, and the sixth shows the P value, between the two odds ratio estimates.

The final column shows the network meta-analysis results, which combine the direct and indirect evidence.

$\mathrm{Cl}$ : confidence interval

IQR: interquartile range

NA: no data available

OR: odds ratio

TTFM: time-to-first mobilisation 
Table 4. Inconsistency table for death at 3 months

\begin{tabular}{lllllll}
\hline $\begin{array}{l}\text { TTFM cate- } \\
\text { gory }\end{array}$ & $\begin{array}{l}\text { TTFM recorded in } \\
\text { the trials (medi- } \\
\text { an; IQR) }\end{array}$ & $\begin{array}{l}\text { Direct } \\
\text { compari- } \\
\text { son } \\
\text { (OR) }\end{array}$ & $\begin{array}{l}\text { Indirect } \\
\text { compari- } \\
\text { son } \\
\text { (OR) }\end{array}$ & $\begin{array}{l}\text { Log difference (95\% CI) } \\
\text { between direct and indi- } \\
\text { rect comparisons }\end{array}$ & $\begin{array}{l}\text { P value of } \\
\text { difference } \\
\text { between di- } \\
\text { rect and in- } \\
\text { direct com- } \\
\text { parisons }\end{array}$ & $\begin{array}{l}\text { Network meta- } \\
\text { analysis } \\
\text { (OR and 95\% CI) }\end{array}$ \\
\hline 12 hours & $13(9$ to 26$)$ & NA & 4.18 & NA & NA & $4.17(0.57$ to 30.7$)$ \\
\hline 18 hours & $18(13$ to 21$)$ & 1.25 & 4.35 & $1.25(-1.16$ to 3.66) & 0.31 & $1.27(0.92$ to 1.76$)$ \\
\hline 24 hours & $26(22$ to 29$)$ & 1.00 (refer- & - & - & - & - \\
\hline$>30$ hours & $32(26$ to 40$)$ & 3.19 & 0.82 & $1.36(-2.12$ to 4.84$)$ & 0.44 & $0.96(0.32$ to 2.92$)$ \\
\hline$>48$ hours & $72(68$ to 75$)$ & 1.73 & 0.77 & $0.81(-1.99$ to 3.62$)$ & 0.57 & $1.41(0.41$ to 4.82$)$ \\
\hline
\end{tabular}

The first two columns show the TTFM category plus the actual recorded TTFM for that category.

The next two columns show the odds ratio of a poor outcome for the direct and indirect comparison of the TTFM category, with 24 hours as the reference.

The fifth column shows the log difference, and the sixth shows the $P$ value, between the two odds ratio estimates.

The final column shows the network meta-analysis results, which combine the direct and indirect evidence.

$\mathrm{Cl}$ : confidence interval

IQR: interquartile range

NA: no data available

OR: odds ratio

TTFM: time-to-first mobilisation

Table 5. 'Summary of findings' table for network meta-analysis (NMA)

\begin{tabular}{|c|c|c|c|c|c|c|}
\hline $\begin{array}{l}\text { Intervention } \\
\text { TTFM }\end{array}$ & $\begin{array}{l}\text { Compari- } \\
\text { son TTFM } \\
\text { (reference } \\
\text { treatment) }\end{array}$ & $\begin{array}{l}\text { No. of stud- } \\
\text { ies (partici- } \\
\text { pants) with } \\
\text { direct com- } \\
\text { parison evi- } \\
\text { dence }\end{array}$ & $\begin{array}{l}\text { Direct comparison } \\
\text { evidence } \\
\text { OR }(95 \% \mathrm{CI})\end{array}$ & $\begin{array}{l}\text { Quality } \\
\text { of the ev- } \\
\text { idence } \\
\text { (GRADE) for } \\
\text { direct com- } \\
\text { parisons }\end{array}$ & $\begin{array}{l}\text { Direct plus indirect evi- } \\
\text { dence (NMA) } \\
\text { OR }(95 \% \mathrm{Cl})\end{array}$ & $\begin{array}{l}\text { Quality } \\
\text { of the ev- } \\
\text { idence } \\
\text { (GRADE) for } \\
\text { NMA }\end{array}$ \\
\hline \multicolumn{7}{|l|}{ Poor outcome } \\
\hline 12 hours & 24 hours & 0 & NA & NA & 6.61 (1.36 to 32.1$)$ & Low $\mathrm{a}, \mathrm{b}$ \\
\hline 18 hours & 24 hours & $1(2104)$ & 1.17 (0.99 to 1.39$)$ & Moderate $c$ & 1.07 (0.53 to 2.19$)$ & Low b, c \\
\hline 30 to 48 hours & 24 hours & $1(32)$ & 3.85 (0.86 to 16.7 ) & Low b, e & 2.74 (1.18 to 6.37$)$ & Low b, e \\
\hline $\begin{array}{l}\text { More than } 48 \\
\text { hours }\end{array}$ & 24 hours & $1(167)$ & $0.94(0.42$ to 2.08$)$ & Low d, e & $1.29(0.50$ to 3.37$)$ & Low b, e \\
\hline \multicolumn{7}{|l|}{ Death } \\
\hline 12 hours & 24 hours & 0 & NA & NA & 4.17 (0.57 to 30.7$)$ & Low $\mathrm{a}, \mathrm{b}$ \\
\hline 18 hours & 24 hours & $1(2104)$ & 1.25 (0.90 to 1.72$)$ & Moderate $c$ & $1.27(0.92$ to 1.76$)$ & Low b, c \\
\hline
\end{tabular}


Table 5. 'Summary of findings' table for network meta-analysis (NMA) (Continued)

\begin{tabular}{llllll}
30 to 48 hours & 24 hours & $1(32)$ & $3.03(0.12$ to 100$)$ & Low b, e & $0.96(0.32$ to 2.92$)$ \\
\hline $\begin{array}{l}\text { More than } 48 \\
\text { hours }\end{array}$ & 24 hours & $1(167)$ & $1.75(0.42$ to 7.14$)$ & Low b, e & $1.41(0.41$ to 4.82$)$ \\
\hline
\end{tabular}

\footnotetext{
a Main trial in the loop was small and had missing data

$b$ Downgraded for imprecision

$c$ Based on a single large trial

$d$ Based on single small trial

e Uncertain blinding of follow up
}

\section{AP P E N DICES}

\section{Appendix 1. CENTRAL and DARE search strategy (the Cochrane Library)}

\#1 [mh ^"cerebrovascular disorders"] or [mh "basal ganglia cerebrovascular disease"] or [mh "brain ischemia"] or [mh "carotid artery diseases"] or [mh "cerebral small vessel diseases"] or [mh "intracranial arterial diseases"] or [mh "intracranial embolism and thrombosis"] or [mh "intracranial hemorrhages"] or [mh^^stroke] or [mh "brain infarction"] or [mh^"stroke, lacunar"] or [mh^"vasospasm, intracranial"] or [mh ^"vertebral artery dissection"]

\#2 (stroke* or poststroke or apoplex ${ }^{\star}$ or cerebral next vasc* or brain next vasc ${ }^{\star}$ or cerebrovasc ${ }^{\star}$ or cva* or SAH):ti,ab

\#3 ((brain or cerebr ${ }^{\star}$ or cerebell* or vertebrobasil ${ }^{\star}$ or hemispher* or intracran* or intracerebral or infratentorial or supratentorial or "middle cerebral artery" or MCA* or "anterior circulation" or "posterior circulation" or "basilar artery" or "vertebral artery" or "space-occupying") near $/ 5$ (isch*emi* or infarct* or thrombo* or emboli* or occlus* or hypoxi $\left.\left.{ }^{\star}\right)\right): t i, a b$

\#4 ((brain* or cerebr* or cerebell* or intracerebral or intracran* or parenchymal or intraparenchymal or intraventricular or infratentorial or supratentorial or basal next gangli* or putaminal or putamen or "posterior fossa" or hemispher* or subarachnoid) near/5 (hemorrhag* or haemorrhage* or hematoma* or haematoma* or bleed*)):ti,ab

\#5 [mh ^ hemiplegia] or [mh paresis]

\#6 (hemipleg* or hemipar* or paresis or paretic):ti,ab

\#7 \#1 or \#2 or \#3 or \#4 or \#5 or \#6

$\# 8\left[\mathrm{mh}^{\wedge}\right.$ "bed rest"] or [mh^immobilization] or [mh^^rest]

\#9 (bed rest or bed-rest or bedrest or bed bound or bed-bound or bedbound):ti,ab

\#10 ((confined or restrict ${ }^{\star}$ or immobili*) near/5 bed):ti,ab

\#11 [mh^^"early ambulation"]

\#12 [mh^^"Physical Therapy Modalities"] or [mh^^"Physical Therapy (Specialty)"]

\#13 [mh ^rehabilitation] or [ $\mathrm{mh}^{\wedge}$ "activities of daily living"] or [ $\mathrm{mh}^{\wedge}$ "recovery of function"]

\#14 [mh ^movement] or [mh ^locomotion] or [mh ^walking] or [mh ^"motor activity"]

\#15 [mh^^"exercise movement techniques"] or [mh ^exercise] or [mh^^"exercise therapy"]

\#16 (stroke unit* or "mobility protocol"):ti,ab

$\# 17 \# 12$ or \#13 or \#14 or \#15 or \#16

\#18 [mh^^"time factors"] or [mh^^time] or early:ti,ab

$\# 19 \# 17$ and \#18 


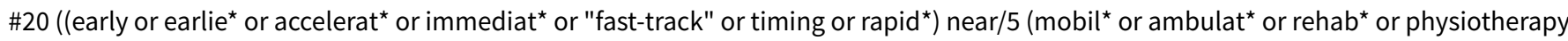
or "physical therapy" or "physical activity" or movement or sitting or standing or walking or semi next recumb* or "out of bed")):ti,ab

$\# 21 \# 8$ or \#9 or \#10 or \#11 or \#19 or \#20

$\# 22 \# 7$ and \#21

\#23 [mh ^"cerebrovascular disorders" [mj]/NU,RH,TH] or [mh "basal ganglia cerebrovascular disease" [mj]/NU,RH,TH] or [mh "brain ischemia" [mj]/NU,RH,TH] or [mh "carotid artery diseases" [mj]/NU,RH,TH] or [mh "cerebral small vessel diseases" [mj]/NU,RH,TH] or [mh "intracranial arterial diseases" [mj]/NU,RH,TH] or [mh "intracranial embolism and thrombosis" [mj]/NU,RH,TH] or [mh "intracranial hemorrhages" [mj]/NU,RH,TH] or [mh ^stroke [mj]/NU,RH,TH] or [mh "brain infarction" [mj]/NU,RH,TH] or [mh "stroke, lacunar" [mj]/ $\mathrm{NU}, \mathrm{RH}, \mathrm{TH}]$ or [mh ^"vasospasm, intracranial" [mj]/NU,RH,TH] or [mh^"vertebral artery dissection" [mj]/NU,RH,TH]

$\# 24 \# 18$ and \#23

\#25 \#22 or \#24

\section{Appendix 2. MEDLINE search strategy}

MEDLINE 2016

1. cerebrovascular disorders/ or exp basal ganglia cerebrovascular disease/ or exp brain ischemia/ or exp carotid artery diseases/ or exp cerebral small vessel diseases/ or exp intracranial arterial diseases/ or exp "intracranial embolism and thrombosis"/ or exp intracranial hemorrhages/ or stroke/ or exp brain infarction/ or stroke, lacunar/ or vasospasm, intracranial/ or vertebral artery dissection/

2. (stroke\$ or poststroke or apoplex\$ or cerebral vasc\$ or brain vasc\$ or cerebrovasc\$ or cva\$ or SAH).tw.

3. ((brain or cerebr\$ or cerebell\$ or vertebrobasil\$ or hemispher\$ or intracran\$ or intracerebral or infratentorial or supratentorial or middle cerebral artery or MCA\$ or anterior circulation or posterior circulation or basilar artery or vertebral artery or space-occupying) adj5 (isch? emi\$ or infarct\$ or thrombo\$ or emboli\$ or occlus\$ or hypoxi\$)).tw.

4. ((brain\$ or cerebr\$ or cerebell\$ or intracerebral or intracran\$ or parenchymal or intraparenchymal or intraventricular or infratentorial or supratentorial or basal gangli\$ or putaminal or putamen or posterior fossa or hemispher\$ or subarachnoid) adj5 (h?emorrhag\$ or h? ematoma\$ or bleed\$)).tw.

5. hemiplegia/ or exp paresis/

6. (hemipleg\$ or hemipar\$ or paresis or paretic).tw.

7. or/1-6

8. bed rest/ or immobilization/ or rest/

9. (bed rest or bed-rest or bedrest or bed bound or bed-bound or bedbound).tw.

10. ((confined or restricted or immobili\$) adj5 bed).tw.

11. early ambulation/

12. Physical Therapy Modalities/ or "Physical Therapy (Specialty)"/

13. rehabilitation/ or "activities of daily living"/ or recovery of function/

14. movement/ or locomotion/ or walking/ or motor activity/

15. exercise movement techniques/ or exercise/ or exercise therapy/

16. (stroke unit\$ or mobility protocol).tw.

17. 12 or 13 or 14 or 15 or 16

18. time factors/ or time/ or early.tw.

19. 17 and 18

20. ((early or earlie\$ or accelerat\$ or immediat\$ or fast-track or timing or rapid\$) adj5 (mobil\$ or ambulat\$ or rehab\$ or physiotherapy or physical therapy or physical activity or movement or sitting or standing or walking or semi-recumb\$ or out of bed)).tw. 
21.8 or 9 or 10 or 11 or 19 or 20

\section{7 and 21}

23. *cerebrovascular disorders/nu, rh or exp *basal ganglia cerebrovascular disease/nu, rh or exp *brain ischemia/nu, rh or exp *carotid artery diseases/nu, rh or exp *cerebral small vessel diseases/nu, rh or exp *intracranial arterial diseases/nu, rh or exp *"intracranial embolism and thrombosis"/nu, rh or exp *intracranial hemorrhages/nu, rh or *stroke/nu, rh or exp *brain infarction/nu, rh or *stroke, lacunar/nu, rh or *vasospasm, intracranial/nu, rh or *vertebral artery dissection/nu, rh

\section{18 and 23}

25. 22 or 24

26. Randomized Controlled Trials as Topic/

27. random allocation/

28. Controlled Clinical Trials as Topic/

29. control groups/

30. clinical trials as topic/

31. double-blind method/

32. single-blind method/

33. randomized controlled trial.pt.

34. controlled clinical trial.pt.

35. clinical trial.pt.

36. (random\$ or RCT or RCTs).tw.

37. (controlled adj5 (trial\$ or stud\$)).tw.

38. (clinical\$ adj5 trial\$).tw.

39. ((control or treatment or experiment\$ or intervention) adj5 (group\$ or subject\$ or patient\$)).tw.

40. (quasi-random\$ or quasi random\$ or pseudo-random\$ or pseudo random\$).tw.

41. ((control or experiment\$ or conservative) adj5 (treatment or therapy or procedure or manage\$)).tw.

42. ((singl\$ or doubl\$ or tripl\$ or trebl\$) adj5 (blind\$ or mask\$)).tw.

43. trial.ti.

44. (assign\$ or allocat\$).tw.

45. controls.tw.

46. 0 r $/ 26-45$

47.25 and 46

48. exp animals/ not humans.sh.

49. 47 not 48

\section{Appendix 3. Embase Ovid search strategy}

1. cerebrovascular disease/ or exp basal ganglion hemorrhage/ or exp brain hematoma/ or exp brain hemorrhage/or exp brain infarction/or exp brain ischemia/or exp carotid artery disease/ or cerebral artery disease/or exp cerebrovascular accident/or exp intracranial aneurysm/ or exp occlusive cerebrovascular disease/ or stroke unit/ or stroke patient/

2. (stroke\$ or poststroke or apoplex\$ or cerebral vasc\$ or brain vasc\$ or cerebrovasc\$ or cva $\$$ or SAH).tw. 
3. ((brain or cerebr\$ or cerebell\$ or vertebrobasil\$ or hemispher\$ or intracran\$ or intracerebral or infratentorial or supratentorial or middle cerebral artery or MCA $\$$ or anterior circulation or posterior circulation or basilar artery or vertebral artery or space-occupying) adj5 (isch? emi\$ or infarct\$ or thrombo\$ or emboli\$ or occlus\$ or hypoxi\$)).tw.

4. ((brain\$ or cerebr\$ or cerebell\$ or intracerebral or intracran\$ or parenchymal or intraparenchymal or intraventricular or infratentorial or supratentorial or basal gangli or putaminal or putamen or posterior fossa or hemispher\$ or subarachnoid) adj5 (h?emorrhag\$ or h? ematoma\$ or bleed\$)).tw.

5. paralysis/ or hemiparesis/ or hemiplegia/ or paresis/

6. (hemipleg\$ or hemipar\$ or paresis or paretic).tw.

7. 1 or 2 or 3 or 4 or 5 or 6

8. bed rest/ or immobilization/ or rest/

9. (bed rest or bed-rest or bedrest or bed bound or bed-bound or bedbound).tw.

10. ((confined or restrict\$ or immobili\$) adj5 bed).tw.

11. mobilization/ or patient mobility/ or physical mobility/

12. exp physiotherapy/ or physiotherapist/ or exp rehabilitation/ or daily life activity/ or convalescence/ or "movement (physiology)"/ or exp locomotion/ or motor activity/ or exp exercise/ or exp kinesiotherapy/

13. (stroke unit\$ or mobility protocol).tw.

14. 11 or 12 or 13

15. early intervention/ or time/ or early.tw.

16. 14 and 15

17. ((early or earlie\$ or accelerat\$ or immediat\$ or fast-track or timing or rapid\$) adj5 (mobil\$ or ambulat\$ or rehab\$ or physiotherapy or physical therapy or physical activity or movement or sitting or standing or walking or semi-recumb\$ or out of bed)).tw.

\section{8 or 9 or 10 or 16 or 17}

19. 7 and 18

20. *cerebrovascular disease/rh or exp *basal ganglion hemorrhage/rh or exp *brain hematoma/rh or exp *brain hemorrhage/rh or exp *brain infarction/rh or exp *brain ischemia/rh or exp *carotid artery disease/rh or *cerebral artery disease/rh or exp *cerebrovascular accident/rh or exp *intracranial aneurysm/rh or exp *occlusive cerebrovascular disease/rh

\section{15 and 20}

\section{19 or 21}

23. Randomized Controlled Trial/ or "randomized controlled trial (topic)"/

\section{Randomization/}

25. Controlled clinical trial/ or "controlled clinical trial (topic)"/

26. control group/ or controlled study/

27. clinical trial/ or "clinical trial (topic)"/ or phase 1 clinical trial/ or phase 2 clinical trial/ or phase $3 \mathrm{clinical}$ trial/ or phase $4 \mathrm{clinical}$ trial/

28. Double Blind Procedure/

29. Single Blind Procedure/ or triple blind procedure/

30. (random\$ or RCT or RCTs).tw.

31. (controlled adj5 (trial\$ or stud\$)).tw.

32. (clinical\$ adj5 trial\$).tw. 
33. ((control or treatment or experiment $\$$ or intervention) adj5 (group\$ or subject\$ or patient\$)).tw.

34. (quasi-random $\$$ or quasi random\$ or pseudo-random $\$$ or pseudo random\$).tw.

35. ((control or experiment\$ or conservative) adj5 (treatment or therapy or procedure or manage\$)).tw.

36. ((singl\$ or doubl\$ or tripl\$ or trebl\$) adj5 (blind\$ or mask\$)).tw.

37. trial.ti.

38. (assign\$ or allocat\$).tw.

39. controls.tw.

40. or/23-39

41. 22 and 40

42. (exp animals/ or exp invertebrate/ or animal experiment/ or animal model/or animal tissue/ or animal cell/ or nonhuman/) not (human/ or normal human/ or human cell/)

\section{41 not 42}

\section{Appendix 4. CINHAL EBSCO search strategy}

S1 .(MH "Cerebrovascular Disorders") OR (MH "Basal Ganglia Cerebrovascular Disease+") OR (MH "Carotid Artery Diseases+") OR (MH "Cerebral Ischemia+") OR (MH "Cerebral Vasospasm") OR (MH "Intracranial Arterial Diseases+") OR (MH "Intracranial Embolism and Thrombosis") OR (MH "Intracranial Hemorrhage+") OR (MH "Stroke") OR (MH "Vertebral Artery Dissections")

\section{S2 .(MH "Stroke Patients") OR (MH "Stroke Units")}

S3 .TI ( stroke* or poststroke or apoplex* or cerebral vasc* or brain vasc* or cerebrovasc* or cva* or SAH ) or AB ( stroke* or poststroke or apoplex* or cerebral vasc ${ }^{\star}$ or brain vasc ${ }^{\star}$ or cerebrovasc ${ }^{\star}$ or $\mathrm{Cva}^{\star}$ or SAH )

S4 .TI ( brain or cerebr ${ }^{\star}$ or cerebell ${ }^{\star}$ or vertebrobasil ${ }^{\star}$ or hemispher ${ }^{\star}$ or intracran* or intracerebral or infratentorial or supratentorial or middle cerebral artery or $\mathrm{MCA}^{\star}$ or anterior circulation or posterior circulation or basilar artery or vertebral artery or space-occupying ) or $\mathrm{AB}$ ( brain or cerebr* or cerebell* or vertebrobasil ${ }^{\star}$ or hemispher* or intracran* or intracerebral or infratentorial or supratentorial or middle cerebral artery or $\mathrm{MCA}^{\star}$ or anterior circulation or posterior circulation or basilar artery or vertebral artery or space-occupying )

S5 . TI (ischemi* or ischaemi or infarct $^{\star}$ or thrombo* or emboli* or occlus* or hypoxi ${ }^{\star}$ ) or AB (ischemi ${ }^{\star}$ or ischaemi ${ }^{\star}$ or infarct $^{\star}$ or thrombo* or emboli* or occlus ${ }^{\star}$ or hypox ${ }^{\star}$ )

\section{S6.S4 and S5}

S7 .TI ( brain* or cerebr* or cerebell* or intracerebral or intracran* or parenchymal or intraparenchymal or intraventricular or infratentorial or supratentorial or basal gangli* or putaminal or putamen or posterior fossa or hemispher* or subarachnoid ) or AB (brain* or cerebr* or cerebell $^{*}$ or intracerebral or intracran* or parenchymal or intraparenchymal or intraventricular or infratentorial or supratentorial or basal gangli* or putaminal or putamen or posterior fossa or hemispher* or subarachnoid)

S8 .TI ( haemorrhage* or hemorrhage* or haematoma* or hematoma* or bleed ${ }^{\star}$ ) or AB ( haemorrhage* or hemorrhage ${ }^{\star}$ or haematoma* or hematoma* or bleed* $)$

S9.S7 and S8

S10.(MH "Hemiplegia")

S11 .TI ( hemipleg* or hemipar* or paresis or paretic ) or AB ( hemipleg* or hemipar* or paresis or paretic)

\section{S12 .S1 OR S2 OR S3 OR S6 OR S9 OR S10 OR S11}

\section{S13.(MH "Early Ambulation")}

S14 .(MH "Bed Rest") OR (MH "Bed Rest Care (lowa NIC)") OR (MH "Rest (lowa NOC)")

S15.(MH "Immobilization")

S16 TI ( bed rest or bed-rest or bedrest or bed bound or bed-bound or bedbound) OR AB ( bed rest or bed-rest or bedrest or bed bound or bed-bound or bedbound) 
S17 .TI ((confined or restricted or immobili*) N5 bed) OR AB ((confined or restricted or immobili $)$ N5 bed)

S18.(MH "Physical Mobility") OR (MH "Mobility Therapy (Saba CCC)")

S19.(MH "Ambulation Aids+") OR (MH "Ambulation Therapy (Saba CCC)") OR (MH "Exercise Therapy: Ambulation (Iowa NIC)") OR (MH "Ambulation: Walking (lowa NOC)") OR (MH "Walking+")

S20.(MH "Activities of Daily Living+") or (MH "Physical Therapy+") or (MH "Rehabilitation") or (MH "Movement+")

S21 .TI ( stroke unit* or mobility protocol ) OR AB ( stroke unit* or mobility protocol )

\section{S22 .S18 OR S19 OR S20 OR S21}

S23.(MH "Time+") OR (MH "Early Intervention") OR TI ( early ) OR AB ( early )

\section{S24 .S22 AND S23}

S25 .TI ( (early or earlie* or accelerat* or immediate* or fast-track or timing or rapid) N10 (mobil* or ambulat* or rehab* or physiotherapy or physical therapy or physical activity or movement or sitting or standing or walking or semi-recumb* or out of bed) ) OR AB ( (early or earlie* $^{\star}$ or accelerat* or immediate or fast-track or timing or rapid) N10 (mobil* or ambulat* or rehab* or physiotherapy or physical therapy or physical activity or movement or sitting or standing or walking or semi-recumb* or out of bed))

\section{S26.S13 OR S14 OR S15 OR S16 OR S17 OR S24 OR S25}

\section{S27.S12 AND S26}

S28.(MH "Randomized Controlled Trials") or (MH "Random Assignment") or (MH "Random Sample+")

S29 .(MH "Clinical Trials") or (MH "Intervention Trials") or (MH "Therapeutic Trials")

S30.(MH "Double-Blind Studies") or (MH "Single-Blind Studies") or (MH "Triple-Blind Studies")

S31.(MH "Control (Research)") or (MH "Control Group")

S32.(MH "Quasi-Experimental Studies")

S33 .PT (clinical trial or randomized controlled trial)

S34 .TI (random* or RCT or RCTs) or AB (random* or RCT or RCTs)

S35 .TI (controlled N5 (trial* or stud*)) or AB (controlled N5 (trial* or stud*))

S36.TI (clinical* N5 trial $\left.^{\star}\right)$ or AB (clinical ${ }^{\star}$ N5 trial $\left.{ }^{\star}\right)$

S37 .TI ((control or treatment or experiment* or intervention) N5 (group* or subject* or patient $\left.{ }^{\star}\right)$ ) or AB ((control or treatment or experiment ${ }^{\star}$ or intervention) N5 (group ${ }^{\star}$ or subject $^{\star}$ or patient $\left.^{\star}\right)$ )

S38 .TI ((control or experiment ${ }^{\star}$ or conservative) N5 (treatment or therapy or procedure or manage $)$ ) or AB ((control or experiment ${ }^{\star}$ or conservative) N5 (treatment or therapy or procedure or manage $\left.{ }^{\star}\right)$ )

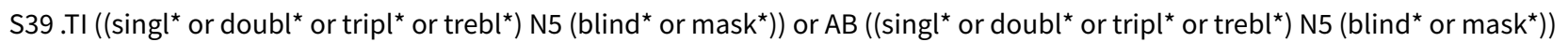

S40.TI trial

S41 .TI (assign* or allocat*) or AB (assign* or allocat*)

S42.TI controls or AB controls

S43 .TI (quasi-random* or quasi random* or pseudo-random* or pseudo random) or AB (quasi-random or quasi random or pseudorandom* or pseudo random*)

S44 .S28 OR S29 OR S30 OR S31 OR S32 OR S33 OR S34 OR S35 OR S36 OR S37 OR S38 OR S39 OR S40 OR S41 OR S42 OR S43

S45.S27 AND S44 


\section{Appendix 5. PsycINFO Ovid search strategy}

1. cerebrovascular disorders/ or cerebral hemorrhage/ or exp cerebral ischemia/ or cerebral small vessel disease/ or cerebrovascular accidents/ or subarachnoid hemorrhage/

2. (stroke or poststroke or apoplex\$ or cerebral vasc\$ or brain vasc\$ or cerebrovasc\$ or cva\$ or SAH).tw.

3. ((brain or cerebr\$ or cerebell\$ or vertebrobasil\$ or hemispher\$ or intracran\$ or intracerebral or infratentorial or supratentorial or middle cerebral artery or MCA\$ or anterior circulation or posterior circulation or basilar artery or vertebral artery or space-occupying) adj5 (isch? emi\$ or infarct\$ or thrombo or emboli\$ or occlus\$ or hypoxi\$)).tw.

4. ((brain\$ or cerebr\$ or cerebell\$ or intracerebral or intracran\$ or parenchymal or intraparenchymal or intraventricular or infratentorial or supratentorial or basal gangli\$ or putaminal or putamen or posterior fossa or hemispher\$ or subarachnoid) adj5 (h?emorrhag\$ or h? ematoma\$ or bleed\$)).tw.

5. hemiparesis/ or hemiplegia/

6. (hemipleg\$ or hemipar\$ or paresis or paretic).tw.

7. or/1-6

8. (bed rest or bed-rest or bedrest or bed bound or bed-bound or bedbound).tw.

9. ((confined or restricted or immobili\$̦) adj5 bed).tw.

10. physical mobility/ or physical therapy/ or physical activity/ or physical therapists/

11. rehabilitation/ or "activities of daily living"/

12. motor processes/ or locomotion/ or walking/

13. exercise/

14. (stroke unit\$ or mobility protocol).tw.

15.10 or 11 or 12 or 13 or 14

16. early intervention/ or time/ or early.tw.

\section{15 and 16}

18. ((early or earlie\$ or accelerat\$ or immediat\$ or fast-track or timing or rapid\$) adj5 (mobil\$ or ambulat\$ or rehab\$ or physiotherapy or physical therapy or physical activity or movement or sitting or standing or walking or semi-recumb\$ or out of bed)).tw.

\section{8 or 9 or 17 or 18}

\section{7 and 19}

21. clinical trials/ or treatment effectiveness evaluation/ or placebo/

22. (random\$ or RCT or RCTs).tw.

23. (controlled adj5 (trial\$ or stud\$)).tw.

24. (clinical\$ adj5 trial\$).tw.

25. ((control or treatment or experiment\$ or intervention) adj5 (group\$ or subject\$ or patient\$)).tw.

26. (quasi-random\$ or quasi random\$ or pseudo-random\$ or pseudo random\$).tw.

27. ((control or experiment\$ or conservative) adj5 (treatment or therapy or procedure or manage\$)).tw.

28. ((singl\$ or doubl\$ or tripl\$ or trebl\$) adj5 (blind\$ or mask\$)).tw.

29. trial.ti.

30. (assign\$ or allocat\$).tw. 
31. controls.tw.

32. or $/ 21-31$

33. 20 and 32

\section{Appendix 6. AMED Ovid search strategy}

1. cerebrovascular disorders/or cerebral hemorrhage/ or cerebral infarction/or cerebral ischemia/ or cerebrovascular accident/ or stroke/

2. (stroke\$ or poststroke or apoplex\$ or cerebral vasc\$ or brain vasc\$ or cerebrovasc\$ or cva\$ or SAH).tw.

3. ((brain or cerebr\$ or cerebell\$ or vertebrobasil\$ or hemispher\$ or intracran\$ or intracerebral or infratentorial or supratentorial or middle cerebral artery or MCA $\$$ or anterior circulation or posterior circulation or basilar artery or vertebral artery or space-occupying) adj5 (isch? emi\$ or infarct\$ or thrombo or emboli\$ or occlus\$ or hypoxi\$)).tw.

4. ((brain\$ or cerebr\$ or cerebell\$ or intracerebral or intracran\$ or parenchymal or intraparenchymal or intraventricular or infratentorial or supratentorial or basal gangli\$ or putaminal or putamen or posterior fossa or hemispher\$ or subarachnoid) adj5 (h?emorrhag\$ or h? ematoma\$ or bleed\$)).tw.

5. hemiplegia/ or gait disorders/

6. (hemipleg\$ or hemipar\$ or paresis or paretic).tw.

7.1 or 2 or 3 or 4 or 5 or 6

8. bed rest/ or immobilization/ or rest/

9. (bed rest or bed-rest or bedrest or bed bound or bed-bound or bedbound).tw.

10. ((confined or restricted or immobili\$) adj5 bed).tw.

11. mobilisation/

12. physical therapy modalities/or physiotherapists/or exp exercise therapy/or rehabilitation/ or exp locomotion/or movement/ or motor activity/ or "activities of daily living"/

13. (stroke unit\$ or mobility protocol).tw.

14. 11 or 12 or 13

15. time/ or early.tw.

16. 14 and 15

17. ((early or earlie\$ or accelerat\$ or immediat\$ or fast-track or timing or rapid\$) adj5 (mobil\$ or ambulat\$ or rehab\$ or physiotherapy or physical therapy or physical activity or movement or sitting or standing or walking or semi-recumb\$ or out of bed)).tw.

18. 8 or 9 or 10 or 16 or 17

19. 7 and 18

20. clinical trials/ or randomized controlled trials/ or random allocation/

21. research design/ or comparative study/

22. double blind method/ or single blind method/

23. (random\$ or RCT or RCTs).tw.

24. (controlled adj5 (trial\$ or stud\$)).tw.

25. (clinical\$ adj5 trial\$).tw.

26. ((control or treatment or experiment\$ or intervention) adj5 (group\$ or subject\$ or patient\$)).tw.

27. (quasi-random\$ or quasi random\$ or pseudo-random\$ or pseudo random\$).tw. 
28. ((control or experiment\$ or conservative) adj5 (treatment or therapy or procedure or manage\$)).tw.

29. ((singl\$ or doubl\$ or tripl\$ or trebl\$) adj5 (blind\$ or mask\$)).tw.

30. trial.ti.

31. (assign\$ or allocat\$).tw.

32. controls.tw.

33. or $/ 20-32$

34. 19 and 33

\section{Appendix 7. SPORTDiscus EBSCO search strategy}

S1 .DE "CEREBROVASCULAR disease" OR DE "BRAIN -- Hemorrhage" OR DE "CEREBRAL embolism \& thrombosis" OR DE "STROKE" OR DE "BRAIN -- Wounds \& injuries" OR DE "BRAIN damage"

\section{S2 .DE "CEREBROVASCULAR disease -- Patients"}

S3 .TI ( stroke* or poststroke or apoplex* or cerebral vasc* or brain vasc ${ }^{\star}$ or cerebrovasc ${ }^{\star}$ or cva* or SAH ) or AB ( stroke* or poststroke or apoplex ${ }^{\star}$ or cerebral vasc ${ }^{\star}$ or brain vasc or cerebrovasc $^{\star}$ or $\mathrm{Cva}^{\star}$ or SAH )

S4 .TI ( brain or cerebr ${ }^{\star}$ or cerebell ${ }^{\star}$ or vertebrobasil ${ }^{\star}$ or hemispher ${ }^{\star}$ or intracran* or intracerebral or infratentorial or supratentorial or middle cerebral artery or $\mathrm{MCA}^{\star}$ or anterior circulation or posterior circulation or basilar artery or vertebral artery or space-occupying ) or $\mathrm{AB}$ ( brain or cerebr* or cerebell* or vertebrobasil ${ }^{\star}$ or hemispher* or intracran* or intracerebral or infratentorial or supratentorial or middle cerebral artery or $\mathrm{MCA}^{\star}$ or anterior circulation or posterior circulation or basilar artery or vertebral artery or space-occupying )

S5 .TI ( ischemi ${ }^{\star}$ or ischaemi ${ }^{\star}$ or infarct ${ }^{\star}$ or thrombo* or emboli* or occlus ${ }^{\star}$ or hypoxi ${ }^{\star}$ ) or AB (ischemi ${ }^{\star}$ or ischaemi ${ }^{\star}$ or infarct $^{\star}$ or thrombo $^{\star}$ or emboli* or occlus ${ }^{\star}$ or hypox ${ }^{\star}$ )

\section{S6 .S4 and S5}

S7 . TI ( brain* or cerebr* or cerebell* or intracerebral or intracran* or parenchymal or intraparenchymal or intraventricular or infratentorial or supratentorial or basal gangli* or putaminal or putamen or posterior fossa or hemispher* or subarachnoid ) or AB (brain* or cerebr* or cerebell* or intracerebral or intracran* or parenchymal or intraparenchymal or intraventricular or infratentorial or supratentorial or basal gangli* or putaminal or putamen or posterior fossa or hemispher* or subarachnoid )

S8 .TI ( haemorrhage* or hemorrhage* or haematoma* or hematoma* or bleed ${ }^{\star}$ ) or AB ( haemorrhage* or hemorrhage* or haematoma* or hematoma* or bleed* $)$

S9.S7 and S8

\section{S10.DE "HEMIPLEGIA" OR DE "HEMIPLEGICS" OR DE "GAIT disorders"}

S11 .TI ( hemipleg* or hemipar* or paresis or paretic ) or AB ( hemipleg* or hemipar* or paresis or paretic )

\section{S12.S1 OR S2 OR S3 OR S6 OR S9 OR S10 OR S11}

\section{S13 .DE "REST" OR DE "IMMOBILIZATION (Therapeutics)"}

S14 .TI ( bed rest or bed-rest or bedrest or bed bound or bed-bound or bedbound) OR AB ( bed rest or bed-rest or bedrest or bed bound or bed-bound or bedbound)

S15 .TI ((confined or restricted or immobili*) N5 bed) OR AB ((confined or restricted or immobili*) N5 bed)

S16.DE "PHYSICAL activity" OR DE "PHYSICAL therapists"

S17 .DE "REHABILITATION" OR DE "MEDICAL rehabilitation" OR DE "RECOVERY training" OR DE "MOVEMENT therapy"

S18 .DE "ACTIVITIES of daily living training" OR DE "ACTIVITIES of daily living" OR DE "FUNCTIONAL training"

S19.DE "BODY movement" OR DE "MOVEMENT therapy" OR DE "LOCOMOTION" OR DE "WALKING" OR DE "EXERCISE" OR DE "ARM exercises" OR DE "CHAIR exercises" OR DE "LEG exercises" OR DE "STRENGTH training" OR DE "STRETCHING exercises"

\section{S20.DE "EXERCISE therapy"}


S21 .TI ( stroke unit* or mobility protocol ) OR AB ( stroke unit* or mobility protocol )

\section{S22 .S16 OR S17 OR S18 OR S19 OR S20 OR S21}

S23.TI ( early ) OR AB ( early)

\section{S24.S22 AND S23}

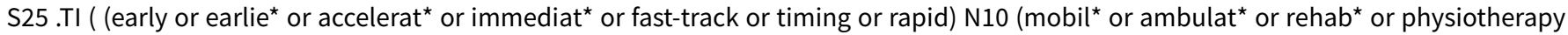
or physical therapy or physical activity or movement or sitting or standing or walking or semi-recumb* or out of bed) ) OR AB ( (early or

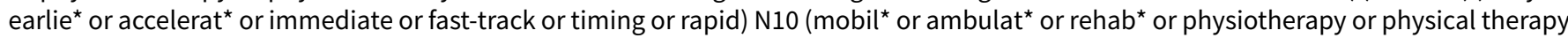
or physical activity or movement or sitting or standing or walking or semi-recumb* or out of bed) )

\section{S26.S13 OR S14 OR S15 OR S24 OR S25}

\section{S27 .S12 AND S26}

\section{Appendix 8. Web of Science search strategy}

Web of Science: Core Collection 1900-2016 (Science Citation Index Expanded (SCI-EXPANDED), Social Sciences Citation Index (SSCI), and Arts \& Humanities Citation Index (A\&HCI)).

\# 26. \#25 AND \#12 AND \#6 (Indexes=SCI-EXPANDED, SSCI, A\&HCI Timespan=2006-2016)

\# 25. \#24 OR \#23 OR \#22 OR \#21 OR \#20 OR \#19 OR \#18 OR \#17 OR \#16 OR \#15 OR \#14 OR \#13

\#24. TS=controls

\# 23. TS=(assign* ${ }^{\star}$ or allocat $\left.{ }^{\star}\right)$

\# 22. TI=trial

\# 21. TS=(placebo* or sham)

\# 20. TS=(cross-over or cross over or crossover)

\# 19 .TS=((singl ${ }^{\star}$ or doubl ${ }^{\star}$ or tripl ${ }^{\star}$ or trebl $\left.{ }^{\star}\right)$ NEAR/5 $\left(\right.$ blind $^{\star}$ or mask $\left.\left.{ }^{\star}\right)\right)$

\# 18. TS=((control or experiment ${ }^{\star}$ or conservative) NEAR/5 (treatment or therapy or procedure or manage*))

\# 17. TS=(quasi-random ${ }^{\star}$ or quasi random ${ }^{\star}$ or pseudo-random* or pseudo random ${ }^{\star}$ )

\# 16. TS=((control or treatment or experiment ${ }^{\star}$ or intervention) NEAR/5 (group ${ }^{\star}$ or subject $^{\star}$ or patient $\left.\left.^{\star}\right)\right)$

\# 15. TS $=\left(\right.$ clinical $^{*} \mathrm{NEAR} / 5$ trial $\left.^{\star}\right)$

\# 14. TS=(controlled NEAR/5 $\left(\right.$ trial $^{\star}$ or stud $\left.\left.{ }^{\star}\right)\right)$

\# 13. TS=(random* or RCT or RCTs)

\# 12. \#7 OR \#8 OR \#11

\# 11. \#9 AND \#10

\# 10. TS=(mobil ${ }^{\star}$ or ambulat* or rehab* $^{\star}$ or physiotherapy or physical therapy or physical activity or movement or sitting or standing or walking or semi-recumb* or out of bed)

\# 9. TS=(early or earlie* or accelerat* ${ }^{\star}$ or immediat* ${ }^{\star}$ or fast-track or timing or rapid ${ }^{\star}$ )

\# 8. TS=((confined or restricted or immobili $\left.{ }^{\star}\right)$ NEAR/5 bed $)$.

\# 7. TS=(bed rest or bed-rest or bedrest or bed bound or bed-bound or bedbound).

\# 6. \#5 OR \#4 OR \#3 OR \#2 OR \#1

\# 5. TS=((unilateral or spatial or hemi\$spatial or visual) NEAR/5 neglect)

\# 4. TS=(hemipleg ${ }^{\star}$ or hemipar ${ }^{\star}$ or paresis or paretic or hemineglect or hemi-neglect) 
\# 3. TS=((brain* or cerebr ${ }^{\star}$ or cerebell ${ }^{\star}$ or intracerebral or intracranial or subarachnoid) NEAR/5 (haemorrhage* or hemorrhage* or haematoma* or hematoma* or bleed $\left.{ }^{\star}\right)$ )

\# 2. TS=((brain* or cerebr ${ }^{\star}$ or cerebell ${ }^{\star}$ or intracran* or intracerebral) NEAR/5 (isch\$emi* or infarct* or thrombo* or emboli* or occlus $\left.\left.{ }^{\star}\right)\right)$

\# 1. TS=(stroke or poststroke or post-stroke or cerebrovasc ${ }^{\star}$ or brain vasc ${ }^{\star}$ or cerebral vasc ${ }^{\star}$ or cva* or apoplex ${ }^{\star}$ or SAH)

WHAT'S NEW

\begin{tabular}{lll}
\hline Date & Event & Description \\
\hline 14 March 2018 & New search has been performed & $\begin{array}{l}\text { Updated literature search with no new completed trials identi- } \\
\text { fied. The review now includes nine RCTs (2958 participants). Text } \\
\text { revised and exploratory network meta-analysis added. }\end{array}$ \\
\hline 14 March 2018 & $\begin{array}{l}\text { New citation required but conclusions } \\
\text { have not changed }\end{array}$ & No change to the conclusions. \\
\hline
\end{tabular}

\section{H I S T ORY}

Protocol first published: Issue 4, 2006

Review first published: Issue 1, 2009

\begin{tabular}{lll}
\hline Date & Event & Description \\
\hline 5 May 2008 & Amended & Converted to new review format. \\
\hline
\end{tabular}

\section{CONTRIBUTIONS OF AUTHORS}

For this review update, Peter Langhorne co-ordinated the updated searches, drafted the update, and re-drafted in response to comments. Peter Langhorne and Trish Bate extracted references. Julie Bernhardt, Janice Collier, Matthew Thuy, and Trish Bate refined the manuscript.

For the original review, Julie Bernhardt drafted the protocol and participated in all stages of the review. Janice Collier and Lynn Legg refined the protocol and contributed to the planned bibliographic searches. Matthew Thuy and Lynn Legg identified studies, assessed methodological quality, and checked the extracted data. Matthew Thuy performed much of the planned bibliographic searches, obtained full-text articles and made contact with study authors. All review authors commented on drafts of the manuscript.

\section{DECLARATIONS OF INTEREST}

Several of the review authors were trialists in at least one of the included trials. However, we allocated trial selection decisions in a manner that avoided trialists making decisions about their own trials.

Peter Langhorne: PL is trialist in two of the included trials (AVERT III 2015; Langhorne 2010). However, trial selection decisions were allocated in a manner to avoid making decisions about his own trials.

Janice M Collier: JC is trialist in two of the included trials (AVERT II 2008; AVERT III 2015). However, trial selection decisions were allocated in a manner to avoid making decisions about her own trials.

Patricia J Bate: none known

Matthew NT Thuy: none known

Julie Bernhardt: JB is trialists in three of the included trials (AVERT II 2008; AVERT III 2015; Langhorne 2010). However, trial selection decisions were allocated in a manner to avoid making decisions about her own trials.

\section{SOURCES OF SUPPORT}

\section{Internal sources}

- No sources of support supplied 


\section{External sources}

- NIHR Priority Review Support Programme, UK.

\section{DIFFERENCES BETWEEN PROTOCOLAND REVIEW}

We added, or more explicitly defined, the following secondary outcomes:

- Death or requiring institutional care: we defined institutional care as care within a residential home, nursing home, or hospital at followup.

- Type of complication (adverse events): categorised as complications of immobility (deep vein thrombosis (DVT), pulmonary embolism $(\mathrm{PE})$, incidence and grade of pressure sores (using standardized grading scale), chest infection, urinary tract infection, falls), and other complications.

- 'Time to walking unassisted (without help from another person) reported alone or as a component of a functional mobility scale' has been replaced by 'Able to walk (Outcome 1.8) and mobility score (Outcome 1.9)'. This minor change was to allow the inclusion of more trial data.

- 'Length of acute stay in acute hospital (Outcome 1.13)' was added to provide an indicator of resource use.

Search Strategy: the WHO Registry now incorporates the Australian Clinical Trials Registry (ACTR; now the Australian New Zealand Clinical Trials Registry (ANZCTR)), the Netherlands Trial Register, and ISRCTNs data sets.

Network meta-analysis: we included an exploratory network meta-analysis in view of the diversity of the included studies.

\section{N DEX TERMS}

\section{Medical Subject Headings (MeSH)}

${ }^{\star}$ Early Ambulation [adverse effects] [mortality]; *Stroke Rehabilitation [mortality]; Activities of Daily Living; Length of Stay; Network Meta-Analysis; Quality of Life; Randomized Controlled Trials as Topic; Time Factors

\section{MeSH check words}

Adult; Humans 